\title{
Simulated Tiltrotor Aircraft Operation in Close Proximity to a Building in Wind and Ground-Effect Conditions
}

\author{
Larry A. Young ${ }^{1}$ \\ NASA Ames Research Center, Moffett Field, CA, 94035
}

\begin{abstract}
A mid-fidelity computational fluid dynamics tool called RotCFD - specifically developed to aid in rotorcraft studies - has been applied to the study of wake interactions of civil tiltrotor aircraft in the immediate vicinity of buildings, in- and out-of-ground-effect, with and without winds, consistent with notional tiltrotor operations from urban vertiports. Such civil tiltrotor operations from urban vertiports could potentially enable city-center-to-citycenter commercial transport. However, in order to one day realize such civil tiltrotor operations, though, it is necessary to better understand the wake interaction and interactional aerodynamic operating environment of urban vertiports. In the early 2000's, a series of 7-by-10 Ft. Wind Tunnel tests were conducted at NASA Ames Research Center that began to explore some of these civil tiltrotor and vertiport wake-interaction/interactionalaerodynamics issues. This study seeks to validate computation fluid dynamic predictions against these early wind tunnel experimental results and, thereby, continue exploration of this important research area.
\end{abstract}

\section{Nomenclature}

$C_{Q} \quad=$ rotor torque coefficient

$C_{T} \quad=$ rotor thrust coefficient

$\mathrm{f}$

$=$ vortex shedding frequency of body, in this case a simulated building

$\mathrm{F}_{\mathrm{z}} \quad=$ tiltrotor test model vertical body force

$\mathrm{h} / \mathrm{R} \quad=$ ratio of rotor height-above-ground with respect to rotor radius

l

$=$ characteristic length of body; in this case the height of a (small-scale) simulated building

$\mathrm{S} \quad=\mathrm{S}=\mathrm{fl} / \mathrm{v}$; Strouhal number of the building wake

$v_{w} \quad=$ simulated wind speed, i.e. wind tunnel freestream velocity; also the characteristic velocity for the Strouhal number estimate

$\mathrm{XTRAV}, \mathrm{x}=$ model absolute longitudinal displacement; origin at building roof-top center/centroid

$\mathrm{x} / \mathrm{R} \quad=$ ratio of right rotor longitudinal-distance from building roof-top center/centroid to the rotor radius

YTRAV, $\mathrm{y}=$ model absolute lateral displacement; origin at building roof-top center/centroid

$\mathrm{y} / \mathrm{R}=$ ratio of right rotor lateral-distance from building roof-top center/centroid to the rotor radius

$\mathrm{z} / \mathrm{R} \quad=$ ratio of right rotor vertical-distance from building roof-top center/centroid to the rotor radius

$\mu \quad$ rotor advance ratio

\section{Introduction}

T has long been envisioned that rotorcraft in general, and tiltrotor aircraft in particular, might one day provide a 1 significant role in commercial transport aviation. Several NASA-sponsored studies over the past two decades have reinforced this vision of commercial transport rotorcraft. In particular, small- to moderate-sized civil tiltrotor (forty or fewer passengers) might be employed to enable city-center-to-city-center flight operations and, thereby, significantly reduce door-to-door transit times for passengers.

\footnotetext{
${ }^{1}$ Aerospace Engineer, Aeromechanics Office, Mail Stop 243-12, AIAA Associate Fellow.
} 
This work is complementary to but distinct from the Ref. 1 computational fluid dynamics (CFD) studies of tiltrotor/vertiport wake interaction effects. The focus of this work is twofold: first, examining some of unique wake interaction of urban vertiports versus airport-sited vertiports and, second, taking advantage of the earlier wind tunnel test data to validate the particular CFD approach taken in this work (and by extension the Ref. 1 work).

\section{Scope of the Problem}

This study will consider validation of computational fluid dynamics results against two sets of complementary small-scale experimental wind tunnel test data. Both wind tunnel tests used test models that were similar to each other; in both cases, attributes of one-forty-eighth-scale V22 tiltrotor aircraft were tested.

\section{Hover and Wind-On In-Ground-Effect}

Reference 2 was primarily focused on tiltrotor aircraft formation flight aerodynamics; however, in the later sections of the paper a small portion of the discussion was focused on aircraft performance measurements inground-effect, during low-speed forward-flight (where the tiltrotor nacelles are in helicopter-mode and the rotors are in edgewise flight). This particular subset of test data has generic applicability to the urban vertiport operation problem. A photograph of the wind tunnel tiltrotor test model is shown in Fig. 1. As can be seen in Fig. 1, the rotors and wing are representative of a one-forty-eighth-scale V22 tiltrotor aircraft but the fuselage - or, rather, the main body of the test model - is not. Instead, the main body of the test model is an assembly of motor, gearbox, servos, a six-component load-measuring balance, and sting adapter. Despite this assembly not being representative of a tiltrotor aircraft, it was assumed in Ref. 2 that the major configuration features were captured to adequately model tiltrotor in-ground-effect aerodynamics. It did, however, present a minor challenge in CFD modeling the astested configuration.

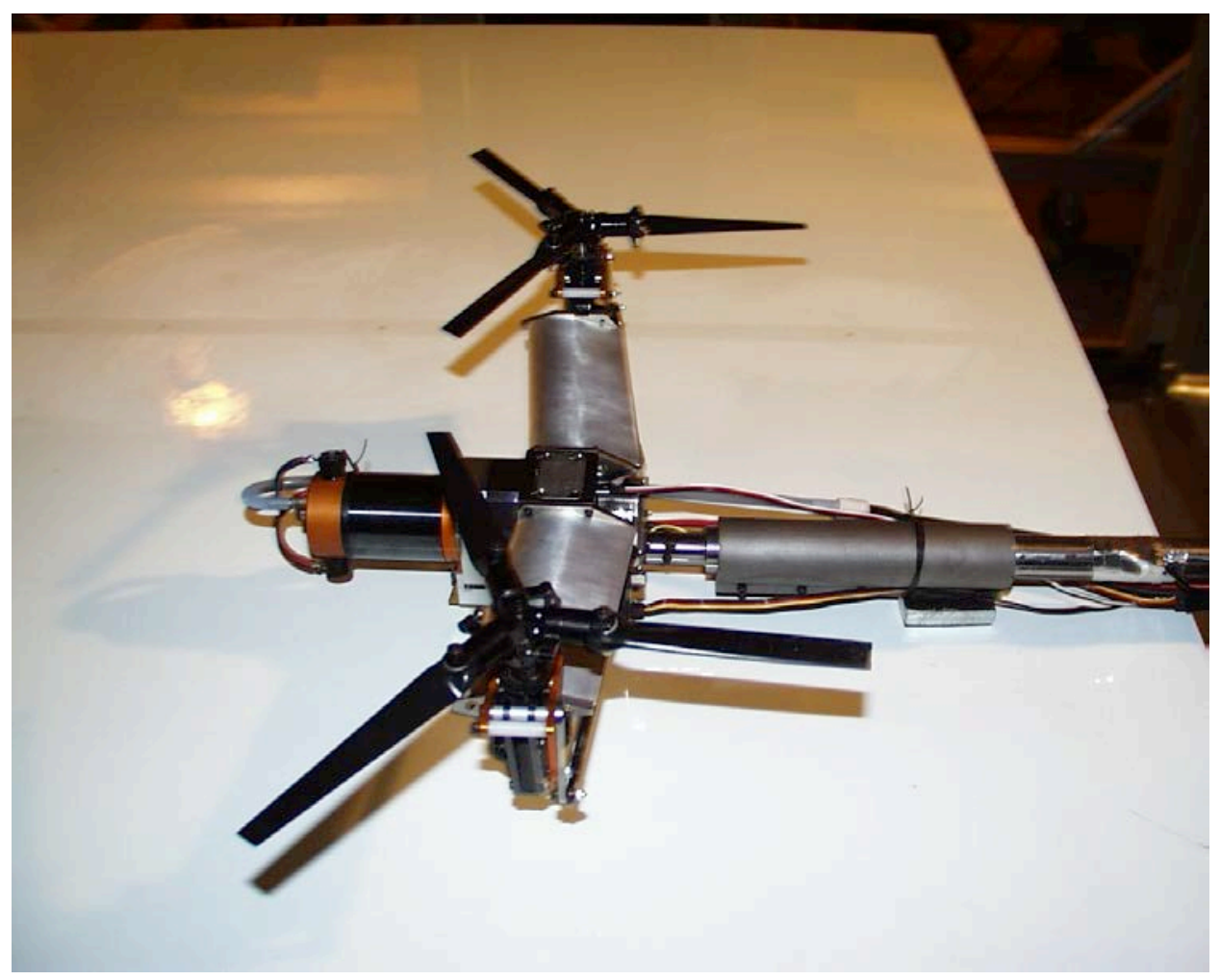

Figure 1. Ref. $21 / 48^{\text {th }}$ scale wind tunnel test model 


\section{“Coy Tower” Problem ${ }^{\dagger}$}

Figure 2 is a photograph of a wind tunnel experiment conducted in the 7-by-10 Ft. test section of the low-speed wind tunnel at NASA Ames Research Center. This test was conducted under the sponsorship of the then-existent NASA Vehicle Systems Program and, specifically, the Runway Independent Aircraft (RIA) project. The emphasis of the experiment was specifically on issues related to civil tiltrotor aircraft in urban city-center operations (aka "urban canyon" interactions). In this regards, Ref. 3 is a direct precursor of the general type of work that the current paper is focused towards. Unfortunately, Ref. 3 has not, to date, been published. The data was provided as a private communication to the author. As such, there are only cursory details available for the experiment. Nonetheless, this experiment is important foundational work and of great value in attempting to establish the predictive capability of computational fluid dynamics software such as the tool used for this study, RotCFD, for complex rotor/wake interaction problems.

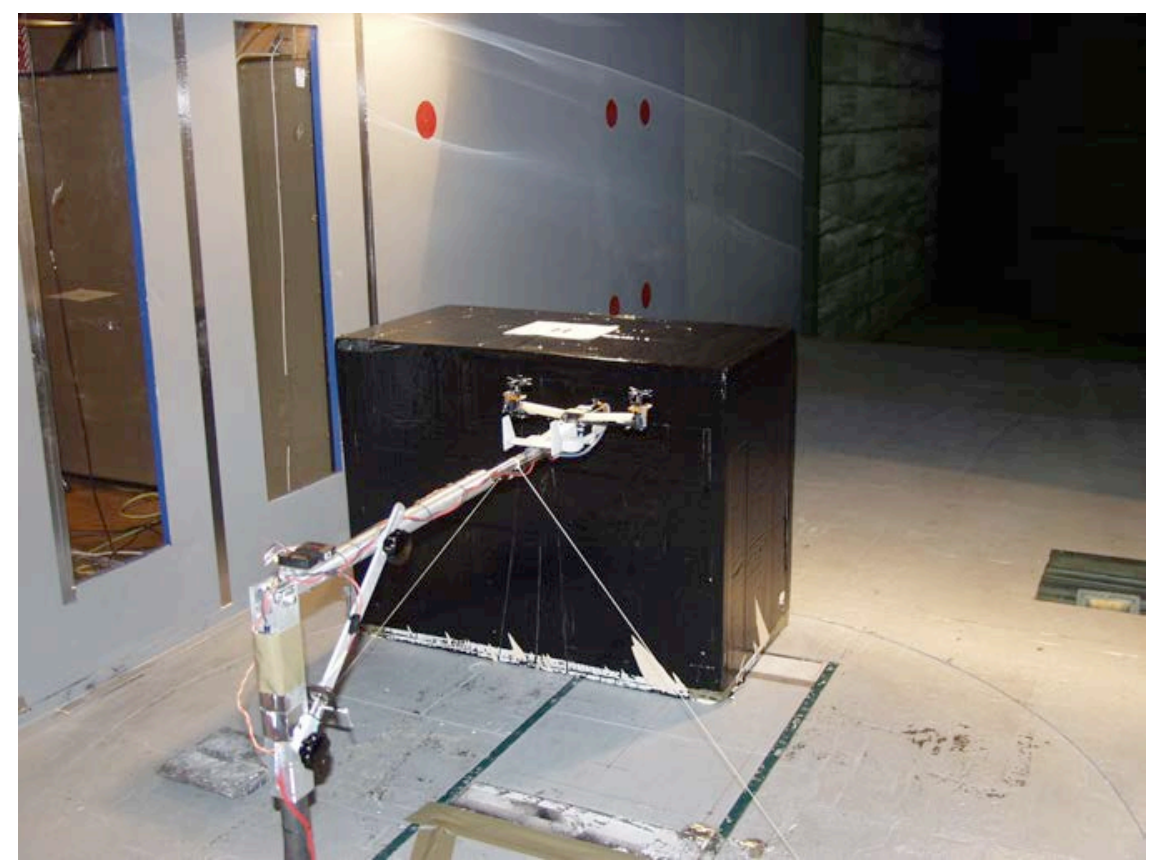

Figure 2. Small-scale Tiltrotor Aircraft Model (Mounted on a Sting)
Downstream of a "Building" in the Wind Tunnel Test Section

Figure 3 is the comparable RotCFD model/gridding of the tiltrotor and building wake interaction experiment shown in Fig. 2. The baseline vehicle design used in this study is a $1 / 48^{\text {th }}$ scaled V-22 geometry. Such nominally scaled V-22 models were employed during wind tunnel test campaigns described in Refs. 2 and 3. The RotCFD modeling does not simulate the wind tunnel mounting hardware used in Refs. 2 and 3 nor the simplifications/modifications - primarily modifications to the fuselage forebody and the empennage to accommodate an electric motor and loading-measuring internal balance - inherent in the test wind tunnel models. Tunnel-wall interference effects are also not modeled, though a ground-plane at the base of the simulated building is included. The origin $(\mathrm{x} / \mathrm{R}=\mathrm{y} / \mathrm{R}=0)$ of the vehicle displacements relative to the building are when the right rotor of the tiltrotor model is directly over the center/centroid of the building roof/top surface; $z / R=0$ would be when the

\footnotetext{
${ }^{\dagger}$ Informally named so after the project manager, at the time, for the Runway Independent Aircraft project, which was the sponsor of the small-scale wind tunnel test.
} 
right rotor is coincident with the building roofline/top, which is, of course, not physically possible. The size of the small-scale simulated building is: height, $\mathrm{H}$, equal to $1.22 \mathrm{~m}$; length (longitudinal dimension with respect to the wind/freestream), L, equal to $1.22 \mathrm{~m}$; and width (lateral dimension), $\mathrm{W}$, equal to $0.61 \mathrm{~m}$. Alternatively, the dimensions can be nondimensionally expressed as $\mathrm{H} / \mathrm{R}=\mathrm{L} / \mathrm{R}=10.23$ and $\mathrm{W} / \mathrm{R}=5.11$.

A Strouhal number of $\mathrm{S}=0.13$ (Ref. 4) was used to estimate a vortex shedding time period for the simulated building wake; given the height $(1.22 \mathrm{~m})$ and the freestream wind tunnel velocity $\left(v_{w}=12.65 \mathrm{fps}\right.$ or $\left.3.86 \mathrm{~m} / \mathrm{s}\right)$, this yielded a time period of 2.43 seconds. The total amount of simulated time in the computational fluid dynamics run cases for this problem was a constant 20 seconds using 2000 time steps. Accordingly, the total simulated time is well above one Strouhal shed vortex cycle. Predicted results for this problem will be presented in two forms: a "last time step" set of numbers and a time-averaged set of numbers for the last 1000 time steps (which should, given the above noted time period estimate reflect 4-5 cycles of the Strouhal-estimated vortex shedding for the simulated small-scale building). The $1 / 48^{\text {th }}$-scale tiltrotor model was modeled with a fine grid; the building had a coarse grid. The rotors were modeled with the "steady" distributed momentum source option; the time step would have had to been decreased by an order of magnitude to exercise the "unsteady rotor" distributed momentum source option. Using the steady distributed momentum source was deemed to be satisfactory for this study.

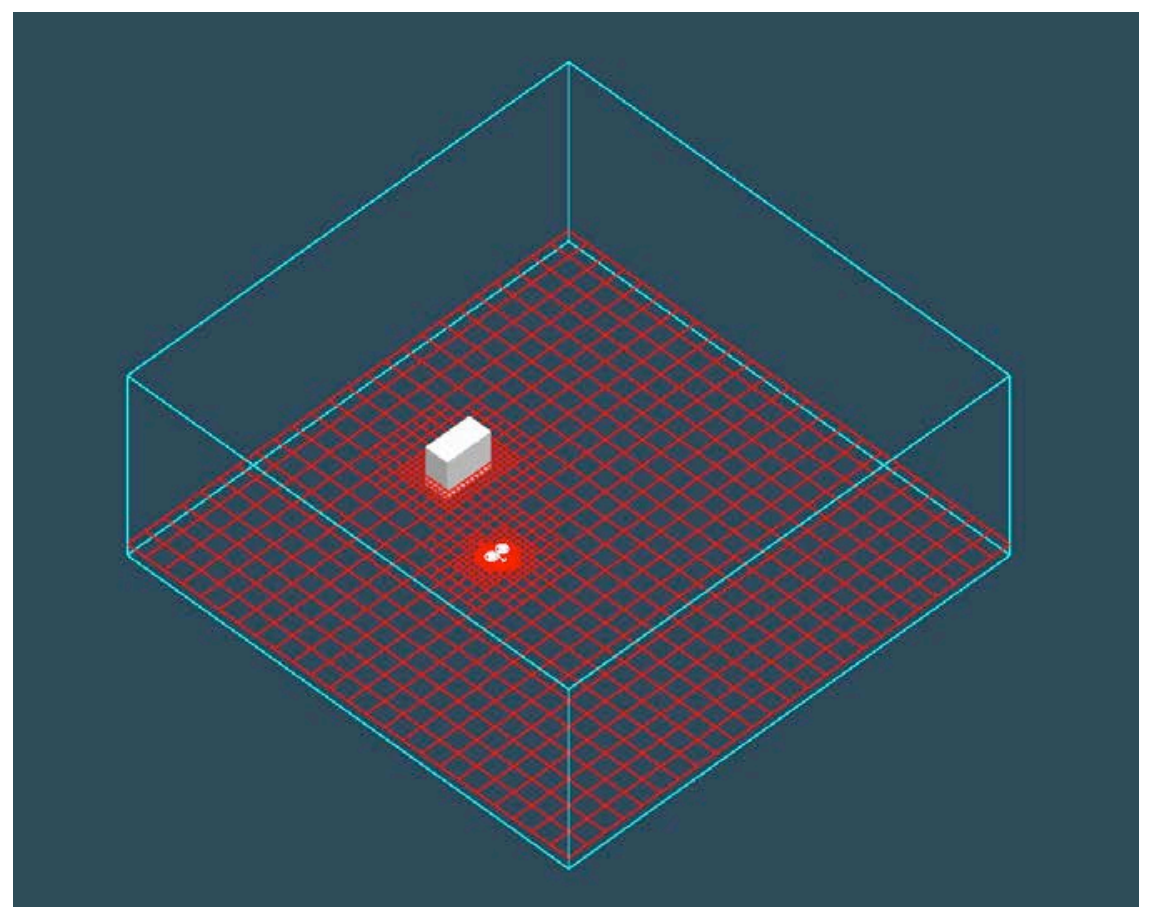

Figure 3. RotCFD Modeling of the Ref. 1 Building/Tiltrotor Wake
Interaction Experiment

The set of low Reynolds number airfoil characteristics (sectional lift and drag coefficients as a function of angle-of-attack and Mach number) used for the rotor momentum source modeling were identical to that used in an earlier study looking at shipboard operations testing using a comprehensive rotorcraft analysis software tool (Ref. 5). The shipboard operations testing and analysis used a very similar one-forty-eighth-scale tiltrotor aircraft (among other test models) as the Ref. 2 test; satisfactory predictions were reported using this airfoil data set. 
Figure 4 presents a representative computational flow field result (velocity magnitude contour plot) from RotCFD. The large bluff-body wake of the simulated building can be clearly see in this figure as well as the downstream interaction of that building wake with the V-22 model.

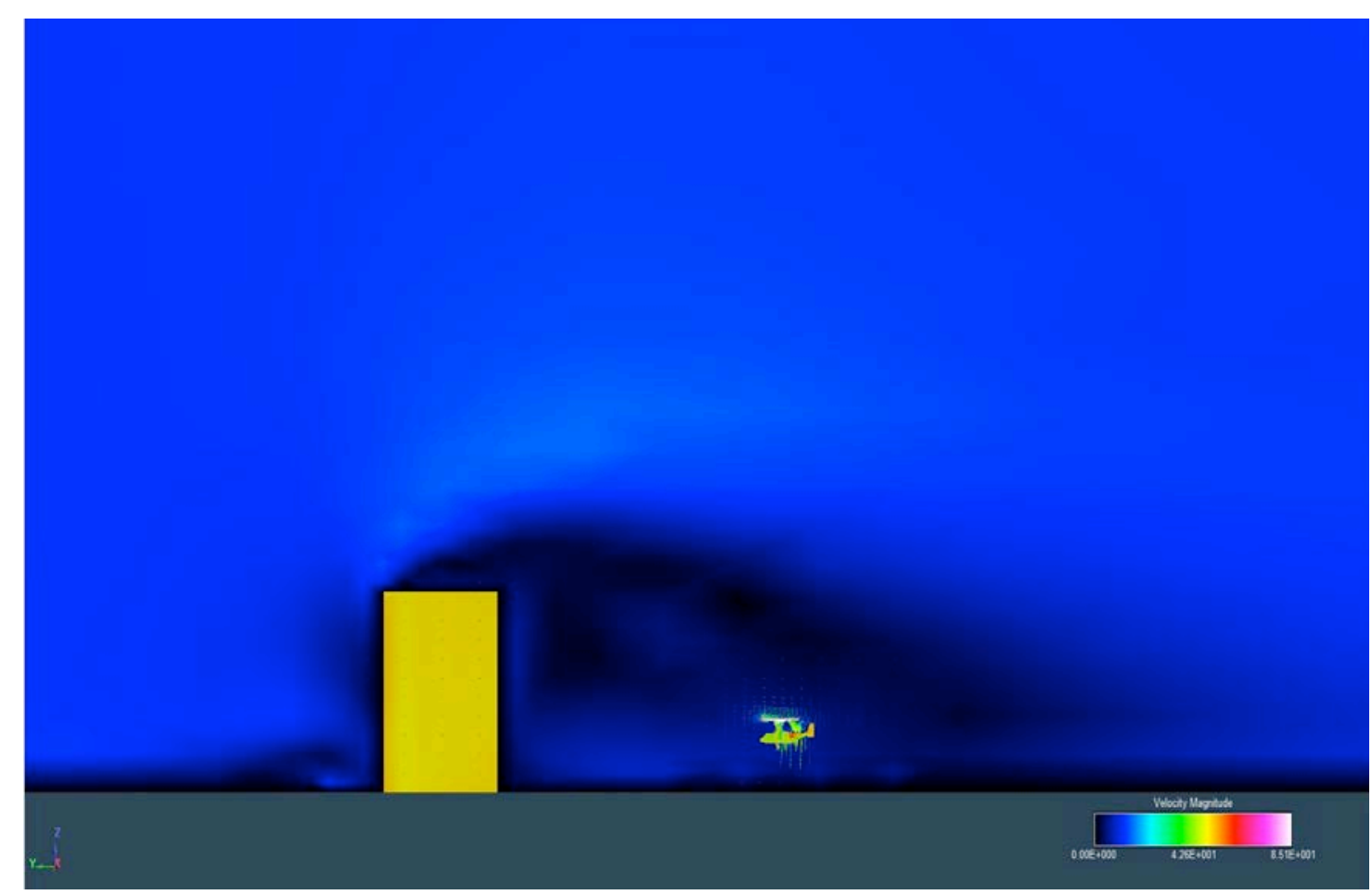

Figure 4. Representative RotCFD flowfield result for the building wake interaction problem

\section{Description of Analysis Approach}

RotCFD attempts to bridge the two worlds of design and computational fluid dynamics (CFD) with the help of an integrated design environment (IDE) specific to rotorcraft. RotCFD emphasizes user-friendliness and efficiency that streamline the design process from geometry to CFD solution. The key components of RotCFD are a geometry module, a semi-automated grid generation module, a flow-solver module, a rotor module, a flow visualization and analysis module, all integrated in one environment. The concept of rotor blades represented by momentum sources forms the basis of this aerodynamic simulation tool for rotorcraft. The rotor momentum sources are primarily a function of the local velocity of the flow and the two-dimensional airfoil characteristics of the rotor blades. The Navier-Stokes equations and the blade element theory are coupled implicitly to yield a self-contained method for generating performance, as well as the near and far wake including all the aerodynamic interference inherent in a situation.

During initial design studies, parametric variation of vehicle geometry is routine. A simple geometry tool (modest CAD functionality) is offered to simplify geometry transformations/changes. The current version of RotCFD can read the body geometry in STL or Plot3D format. Also, some simple shapes (bodies of revolution) can be generated from within RotCFD. In addition to general transformations such as translation, rotation and scaling, parametric variation of the geometry is available to assist with the design. Graphical visualization is used to make 
the geometry manipulation user-friendly: the user is able to see a preview of the geometry change before committing the change.

The SIMPLER/SIMPLE line of pressure-based algorithms (Ref. 6) is suitable for low speed flows and currently SIMPLE is used for solving the flow field in RotCFD. Additional details describing RotCFD are summarized in Ref. 7. The flow predictions for this study were generated using the "realizable $\kappa-\varepsilon$ " turbulence model (Ref. 8); the flow problem was treated as fully turbulent. RotCFD has the option of studying the problem as a fully laminar one, but this was not done for this study; the software does not currently have a laminar-to-turbulent transition model incorporated into it.

\section{Results}

\section{Hover and Wind-On In-Ground-Effect}

During the early 2000's, a series of tiltrotor wake interaction studies were performed in the NASA Ames 7-by10 Ft. low-speed wind tunnel. These wind tunnel tests employed several small-scale $\left(\sim 1 / 48^{\text {th }}\right.$ scale $)$ tiltrotor test models. The majority of these small-scale tiltrotor tests focused on shipboard operations and aircraft formation flight but a few (e.g. Ref. 2 and 3) were directly relevant to the tiltrotor/vertiport wake interaction work of this paper. This section will focus on the performing RotCFD validation against experimental data from Ref. 2.

Reference 2 was primarily focused on tiltrotor aircraft formation flight aerodynamics; however, in the later sections of the paper a small portion of the discussion was focused on aircraft performance measurements inground-effect, during low-speed forward-flight (where the tiltrotor nacelles are in helicopter-mode and the rotors are in edgewise flight). Figure 5(a) is a photograph of the Ref. 2 test model and Fig 5b is an illustration of the forward flight version of the RotCFD model (the hover version of the RotCFD modeling eliminated the nacelles for simplicity).

As noted previously, the current versions of the RotCFD do not have an automated trim function. Therefore, accordingly, in order to match IGE/forward-flight data from Ref. 2 it was necessary to run a rotor collective sweep and then select a collective setting for the nominal CT set point used in the Ref. 2 data set (i.e. a trim condition of $\mathrm{CT}=0.012$ at an advance ratio, $\mu$, of 0.1 ). Figure 6 summarizes the rotor thrust and torque trends as a function of collective for the target advance ratio. Unfortunately, there is no comparable experimental data to validate these predicted performance trends.

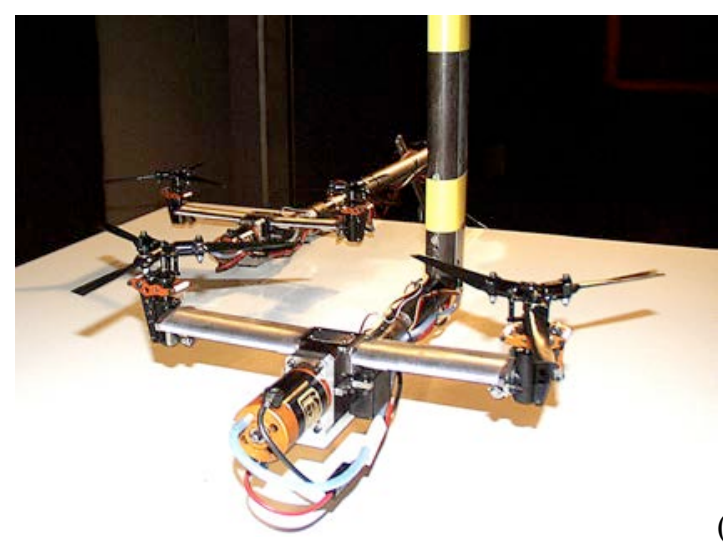

(a)

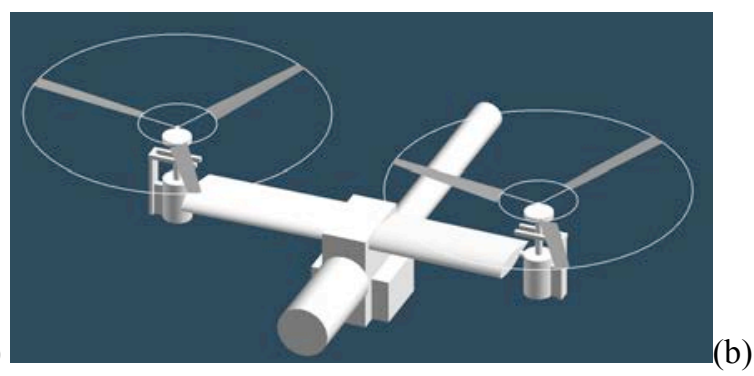


Figure 5. (a) 2002 One-Forty-Eighth-Scale Tiltrotor IGE Testing and (b) RotCFD Modeling

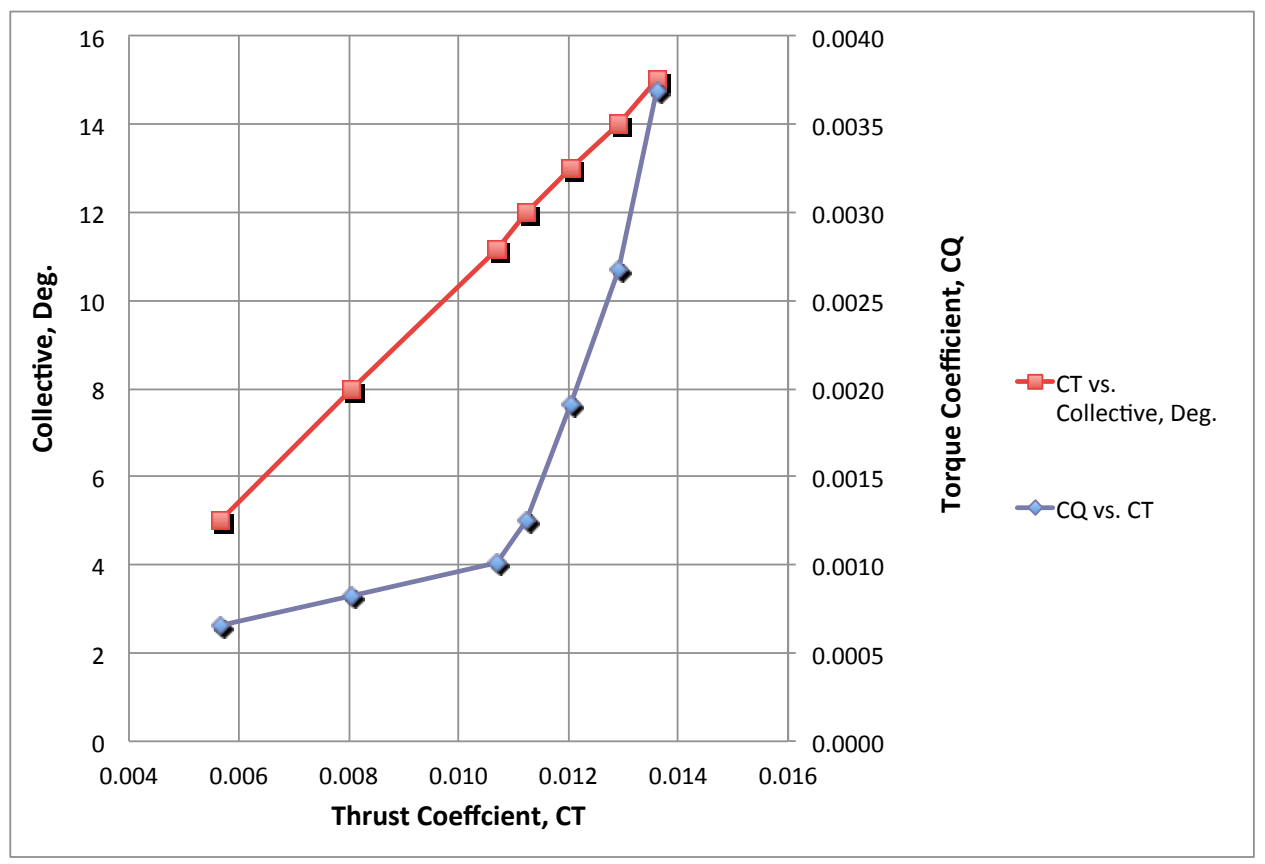

Figure 6. RotCFD Predicted Thrust. Torque, and Collective Sweep at $\mu=0.1$ for the Ref. $21 / 48^{\text {th }}$ scale Tiltrotor Model; used to establish fixed collective setting for height sweeps to match $\mathrm{CT}=0.012$ target value for $\mathrm{Mu}=0.1 \mathrm{OGE}$ condition

Some flow visualization pictures of the wake velocities near the ground are shown in Fig. 7a-c of the tiltrotor test model in ground effect in hover and $\mu=0.05$ and 0.1 . These distinctive crescent-shaped flow patterns are symptomatic of a large horseshoe vortex pattern being formed by the tiltrotor when in ground effect. Similar crescent-shaped wake patterns along ground planes are seen in Ref. 2 china clay and flow tuft photographs. 


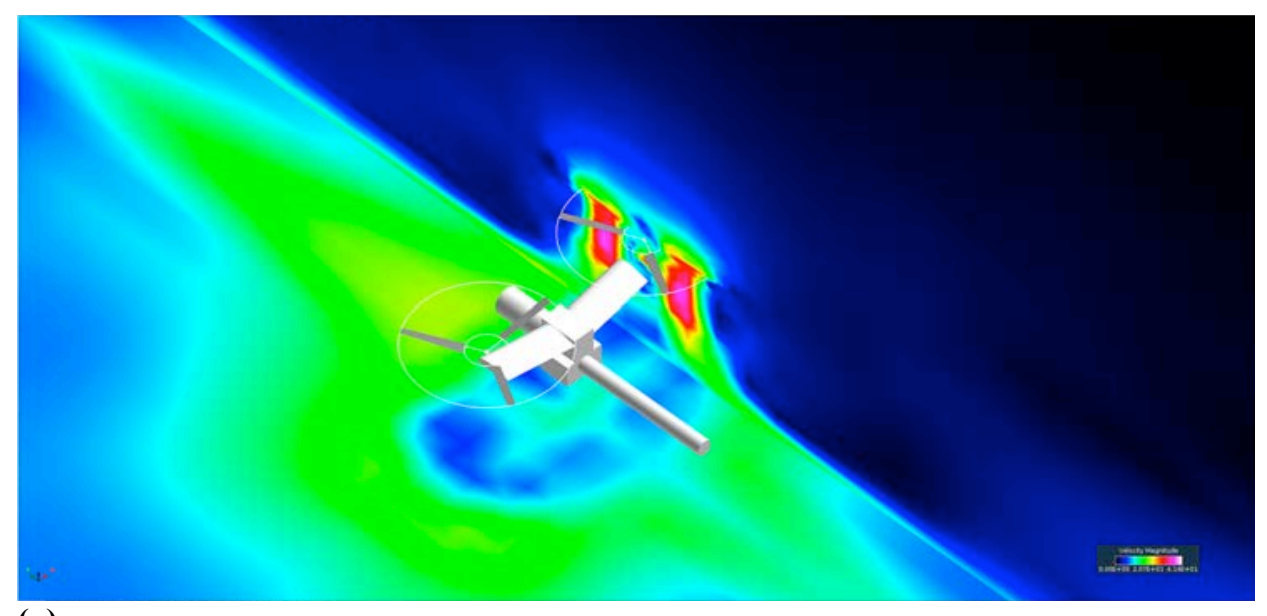

(a)

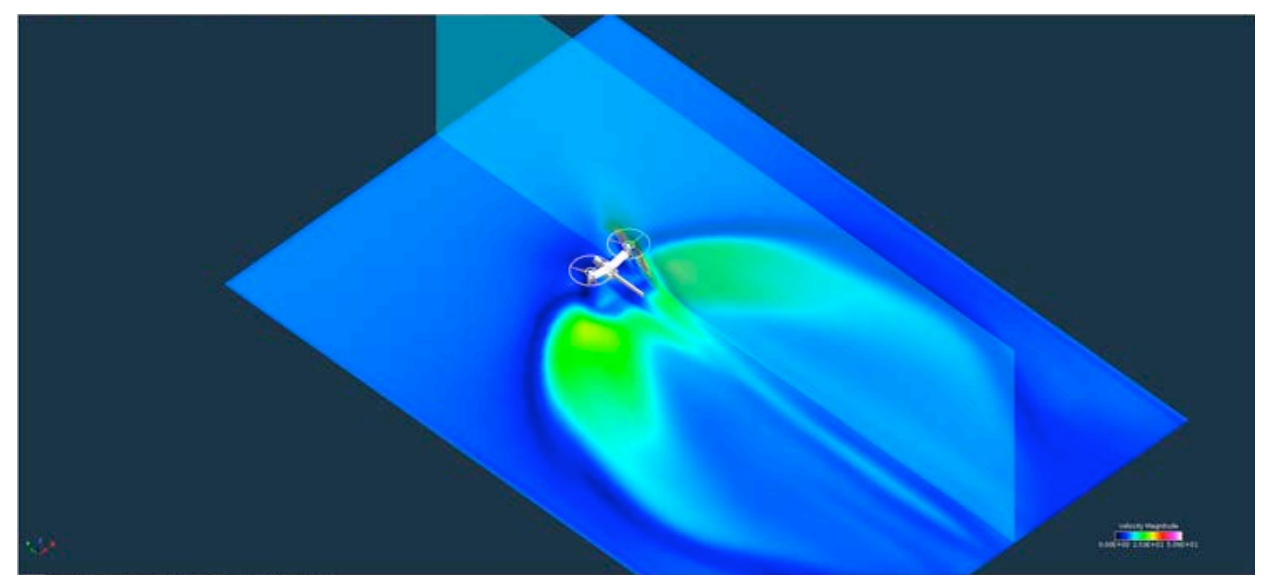

(b)

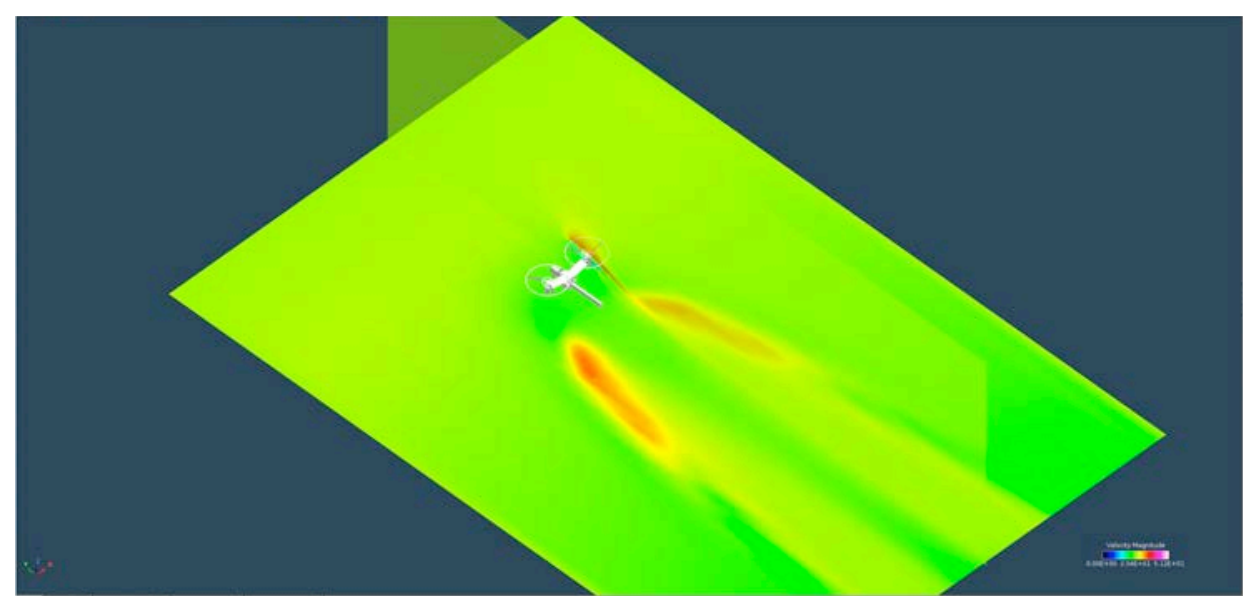

(c)

Figure 7. RotCFD Flow Field Predictions $(h / R=1.79$ and $h / R=2.05$ for $m u=0.1)$ : (a) $\mu=0$; (b) $\mu=0.05$; and (c) $\mu=0.1$. 


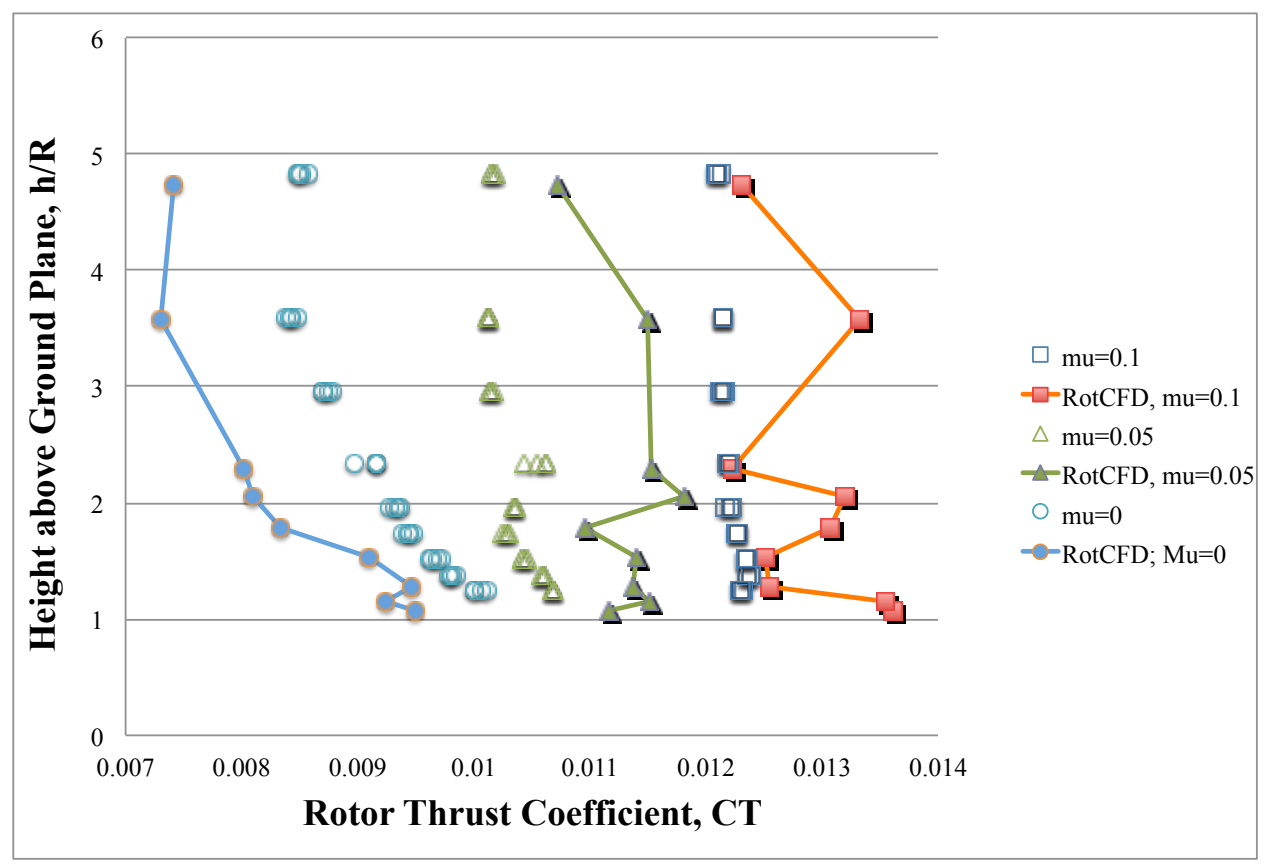

Figure 8. Correlation between Experimental Results and RotCFD Predictions for the Reference 2 1/48'th-scale Tiltrotor Model in Forward-Flight Helicopter-Mode IGE

The Figure 8 agreement between the CFD predictions and the experimental data trend-wise looks generally acceptable but clearly there are constant delta offsets in the overall thrust coefficient magnitudes. A better sense of the general trend agreement between prediction and experiment can be gained by normalizing the thrust coefficient results/data by the out of ground effect thrust coefficient value for a given operating condition, i.e. hover and $\mu-0.05$ and 0.1. This normalized set of results is presented in Fig. 9a-c. Figure 9a-c reveals that in terms of relative in ground effect trend, the CFD results are in good agreement with the experimental data, though the net thrust might be over-predicted for the mid-range of the height sweep, i.e. $2<\mathrm{h} / \mathrm{R}<3$, and the $\mu=0.1$ predictions are showing larger decreasing/increasing zig-zag patterns of thrust variation with height than what is seen in the experimental data. 


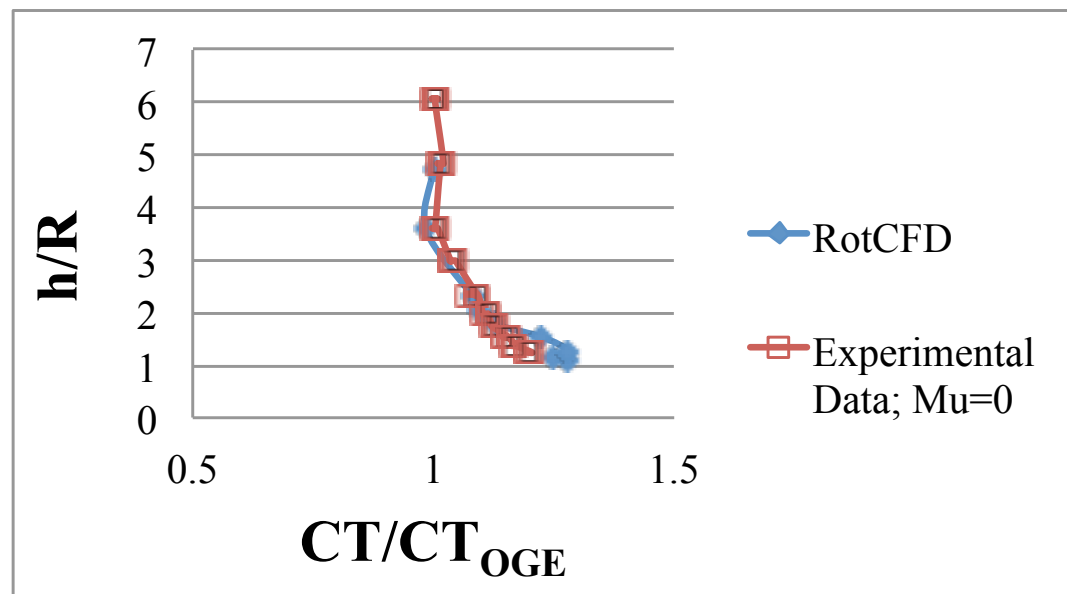

(a)

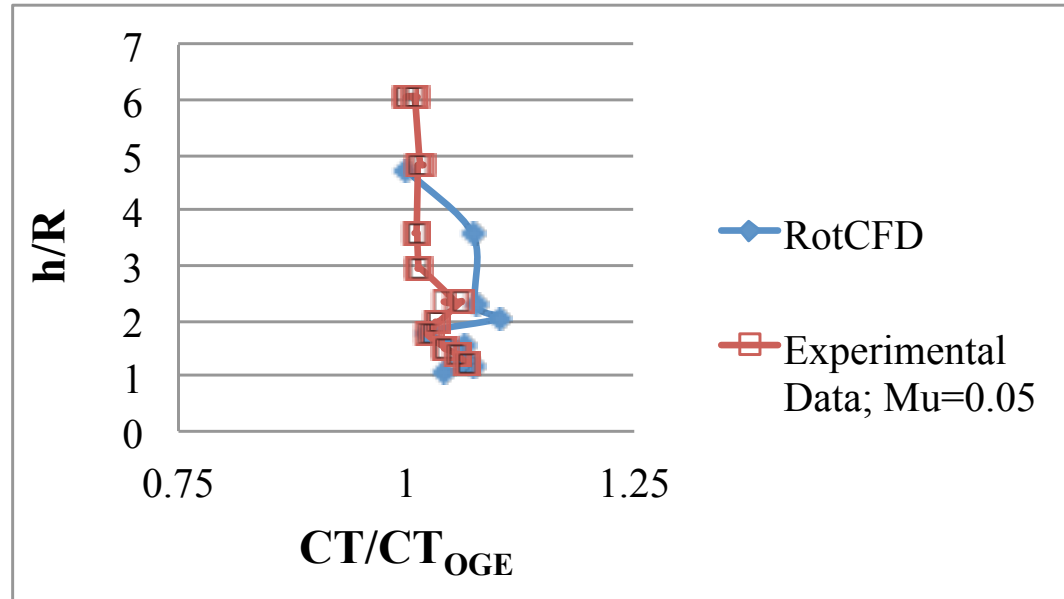

(b)

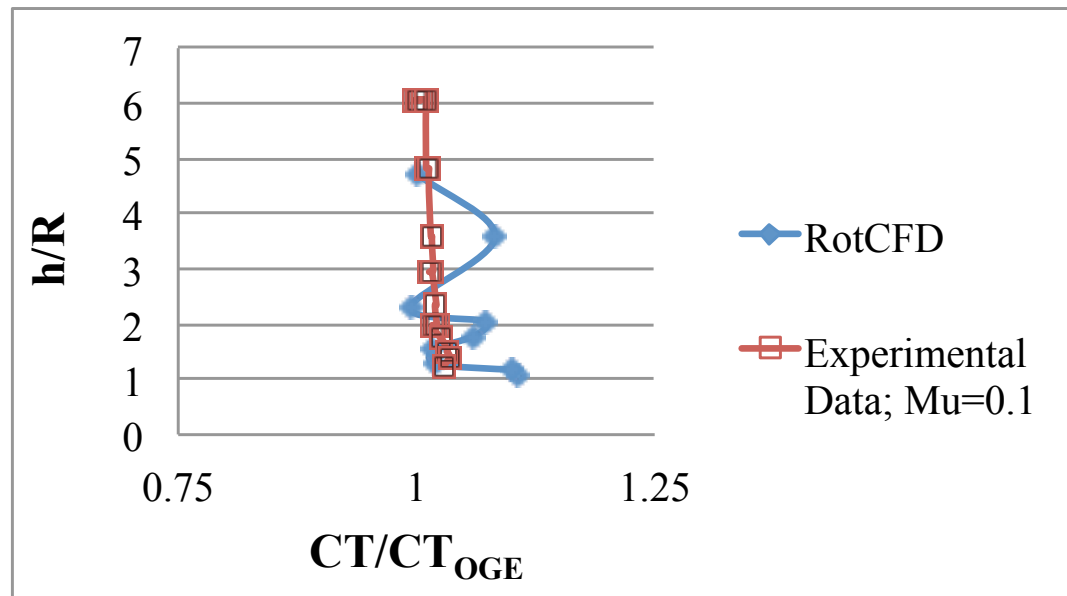

(c)

Figure 9. Normalized agreement: (a) $\mu=0$; (b) $\mu=0.05$; and (c) $\mu=0.1$.

The above in-ground-effect trend agreement is due to accounting for the combined rotor thrust and vertical body force of the test model. The general trend for hover in ground effect for a tiltrotor model, Fig. 9a, can only be 
captured by incorporating the vertical body force of the test model in addition to the rotor contributions to the net thrust/lift of the overall test model; otherwise, if only the rotor contributions are considered then large trend discrepancies result as can be seen in Fig. 10a. It appears, though as wind speed increases that the vertical body force, $F_{z}$, has less of an influence of the height sweep variation on vehicle net lift/thrust. Even some fairly subtle nuances in the in-ground-effect trends such as seen for $\mu=0.05$, are satisfactorily predicted. Note that as $h / R$ becomes very small (in this particular case as $\mathrm{h} / \mathrm{R} \rightarrow 1$ ) there is a small influence as to the lack of proper boundary layer modeling at the ground plane: the thrust level dips a bit in the RotCFD results but not in the hover and $\mu=0.05$ operating conditions.
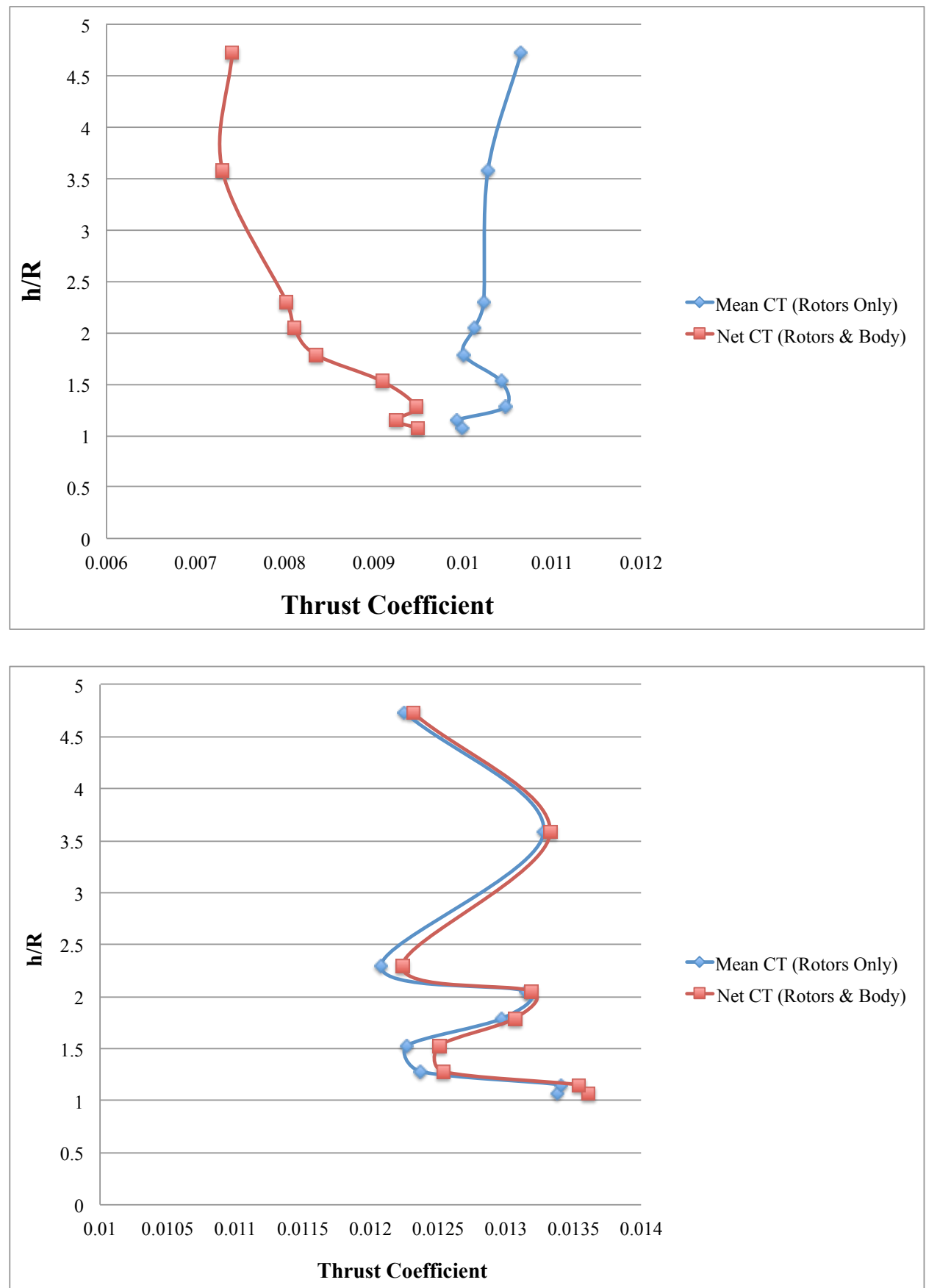

(a)

Figure 10. Test Model in Ground Effect (rotors only contribution versus combined rotors and model vertical body force): (a) hover, $\mu=0$, and (b) low speed forward flight,,$\mu=0.1$ 
Figure 11 provides as sample set of RotCFD generated tiltrotor test model surface pressure distributions inherent in the estimates of the model forces and moments. It is clear from this force and moment results that a significant negative $\mathrm{F}_{\mathrm{z}}$ is being generated on the test model. It is left for future work to determine how representative the Figs. 8-9 trends are - for the Ref. 2 test model - as compared to a more geometrically correct tiltrotor aircraft.
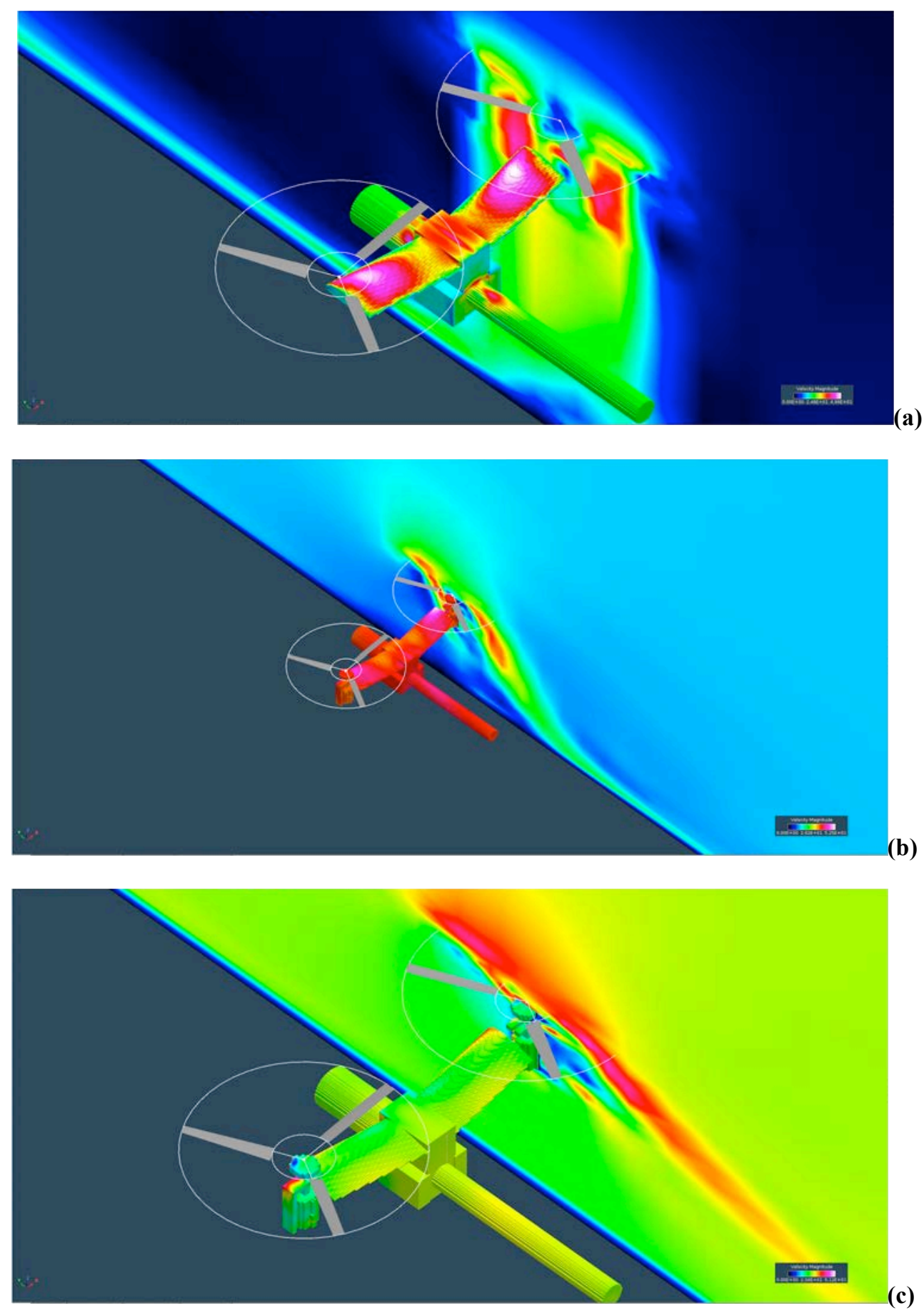

Figure 11. RotCFD Test Model Pressure Distribution Predictions $(h / R=2.05$ and $h / R=3.48$ for $m u=0.1$ ): (a) $\mu=0$; (b) $\mu=0.05$; and (c) $\mu=0.1$. 


\section{Coy Tower Problem}

Three different horizontal survey planes of the modeled tiltrotor with respect to the building and ground were studied. The upper plane was located at $\mathrm{z} / \mathrm{R}=2$ above the simulated building rooftop; the middle plane was located $\mathrm{h} / \mathrm{R}=3.3$ above the ground and the lower plane was located at $\mathrm{h} / \mathrm{R}=1.1$ above the ground. Each horizontal plane of data will be discussed sequentially as follows. The majority of the correlation/validation effort has focused on studying the tiltrotor aerodynamics for the upper plane survey. Table 1 is a summary of the isolated one-fortyeighth-scale V22 tiltrotor model (without the building or its wake interference) rotors' force and moment coefficients (and thrust, $l_{\mathrm{f}}$, and power, $\mathrm{W}$ ) and the model's fuselage forces and moments (in units of $\mathrm{lb}_{\mathrm{f}}$ and $\mathrm{ft}-\mathrm{lb}_{\mathrm{f}}$ in this table of raw output from RotCFD). Note in particular that all fuselage moments for the isolated tiltrotor model are fairly small with the exception of the pitching-moment. The full aerodynamic interactions of rotors-on-fuselage and fuselage-on-rotors is ideally captured in this prediction.

\section{Table $1-1 /$ s8 $^{\text {th }}$ scale Tiltrotor Model Predicted Forces and Moments without Building Wake Interference (Last Time Step of RotCFD Run; table units $\mathrm{lb}_{\mathrm{f}}$ and $\mathbf{f t}-\mathrm{lb}_{\mathrm{f}}$ )}

\begin{tabular}{|c|c|c|c|c|c|c|c|c|c|c|}
\hline Tmsp & Rtr & Thrust & $\mathrm{CT}$ & $\mathrm{CH}$ & $\mathrm{CY}$ & Torque & $\mathrm{CQ}$ & CPM & CYM & Total_Power \\
\hline ------ & ---- & ------------- & -------------- & -------------- & -------------- & -------------- & -------------- & -------------- & -------------- & -------------- \\
\hline 2000 & & $11.12 \mathrm{E}+00$ & $1.42 \mathrm{E}-02$ & 4.77E-04 & $-5.89 E-04$ & 9.35E-02 & 3.04E-03 & $1.51 \mathrm{E}-03$ & 3.65E-04 & $6.30 \mathrm{E}+01$ \\
\hline 2000 & & $21.16 \mathrm{E}+00$ & $1.48 \mathrm{E}-02$ & $9.17 \mathrm{E}-05$ & $-5.43 E-04$ & $-9.34 \mathrm{E}-02$ & $-3.03 E-03$ & $-1.44 \mathrm{E}-03$ & $-2.30 \mathrm{E}-04$ & $6.29 E+01$ \\
\hline Tmsp & Fuselage SF & F Fus. Drag & Fus. Lift & Fus. PM & Fus. RM & Fus. YM & & & & \\
\hline 2000 & $-2.69 \mathrm{E}-04$ & 4 4.64E-02 & $-5.70 \mathrm{E}-02$ & $5.84 \mathrm{E}-01$ & $-7.77 \mathrm{E}-04$ & $2.71 \mathrm{E}-03$ & & & & \\
\hline
\end{tabular}

\section{Upper Survey Plane ( $z / R=2$ above rooftop)}

Figure 12a-e illustrates one partial sweep of the $1 / 48^{\text {th }}$ scale tiltrotor model, where the lateral displacement of the model is varied $(-8<=\mathrm{x}<=0)$. In Fig. $12 \mathrm{a}-\mathrm{e}$, the $1 / 48^{\text {th }}$ scale model is initially partially in quasi-ground-effect with the building rooftop $(\mathrm{y}=1.5$ and $\mathrm{z} / \mathrm{R}=2$ above the building) and then sees a reduction in this rooftop "ground effect" as the vehicle shifts laterally or longitudinally with respect to the rooftop. In the cases where the vehicle is in this partial rooftop "IGE" the rotor wake/outwash significantly interacts with the top of the urban building. Initially (Fig. 5a) the upper surface pressure distributions across the vehicle (particularly as comparing the right versus the left side of the vehicle wing) are symmetrical, when the vehicle is centered directly in the building wake. As the tiltrotor is shifted laterally to the left with respect to the building, an increasing asymmetry of the upper surface pressures can be observed (Fig. 12b-c). Finally as the tiltrotor model is displaced even further (Fig. 12d-e), as the vehicle shifts outside of the building wake and enters primarily uniform freestream velocity (simulating a constant wind profile for all but the base of the building). Unfortunately, though flow field smoke flow visualization was performed during the Ref. 3 wind tunnel test, there are apparently no surviving images of the flow visualization. Future experimental work will need to not only accurately capture the vehicle aerodynamic characteristics but, equally so, must robustly characterize the essentially bluff body flow of simulated buildings. 

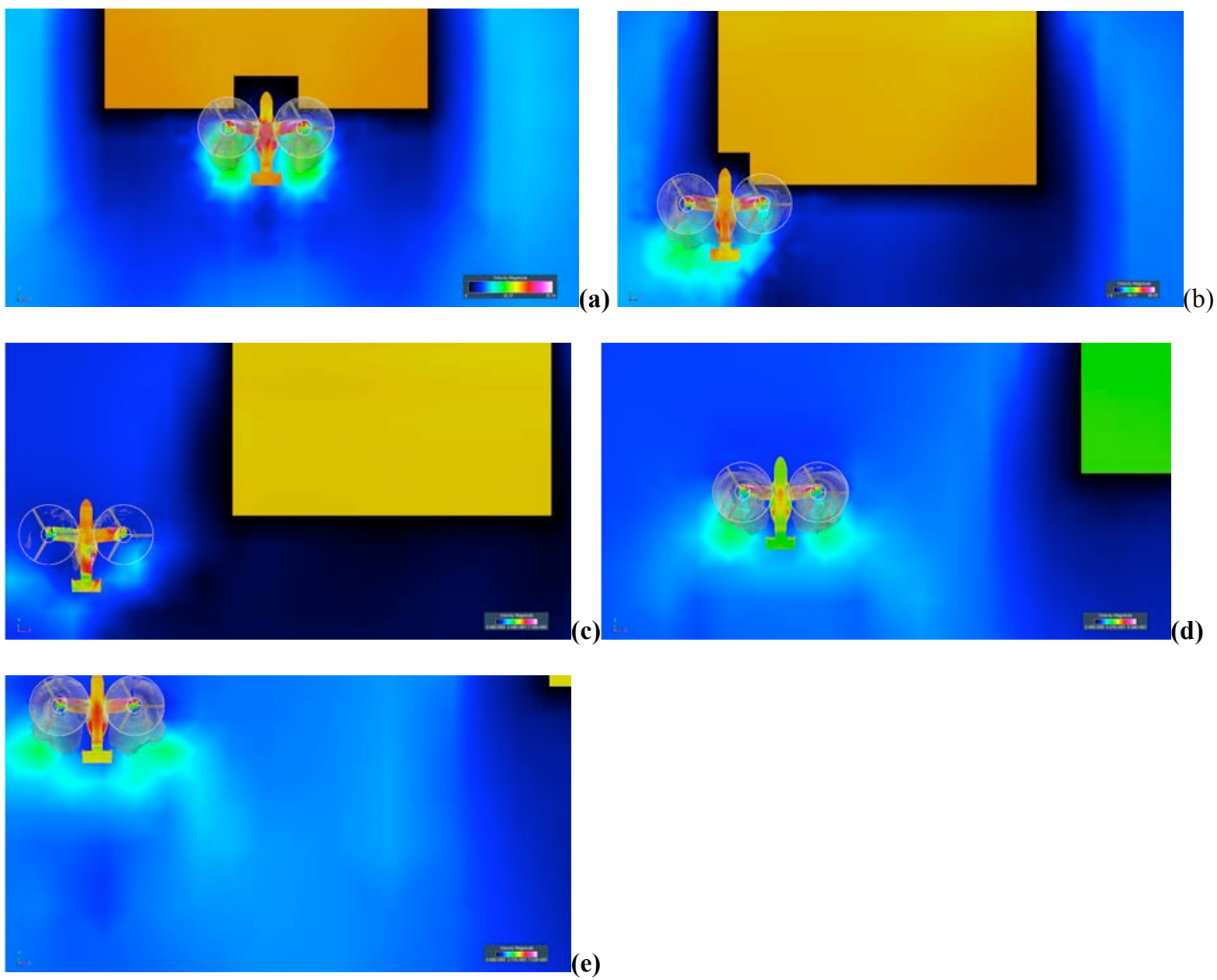

Figure 12. CFD predictions of upper surface pressure distribution (surface pressure scaling not held constant) of $1 / 48^{\text {th }}$ scale model as influence by lateral displacement from the simulated building roof-top $(\mathrm{x} / \mathrm{R}=3.84):$ (a) $\mathrm{y} / \mathrm{R}=\mathbf{0}$; (b) $\mathrm{y} / \mathrm{R}=-\mathbf{5 . 1 1}$; (c) $\mathrm{y} / \mathrm{R}=-10.23$; (d) $\mathrm{y} / \mathrm{R}=-15.35 ;$ (e) $\mathrm{y} / \mathrm{R}=-20.46$

Figure 13 presents estimates of the model electrical supply power as compared to experimental data measured in Ref. 3. RotCFD predicted each rotor's shaft power and electrical supply power was estimated by assuming a motor and gearbox efficiency of 0.8 and 0.9 respectively. In this figure, a trend line for both the power estimates for the last time step (time step $=2000$ at the simulated total time of 20 seconds) and an averaged trend line (for the last 1000 time steps, over a delta simulated time period of 10 seconds). For Fig. 13 the one-forty-eighth-scale V-22 tiltrotor test model variation in electrical supply power as a function of lateral displacement from simulated building rooftop (at a height of $\mathrm{z} / \mathrm{R}=2$ ) in simulated wind condition can be seen. Note that at $\mathrm{y} / \mathrm{R}=0$ the model is directly centered over the building rooftop. The general trends (though not the absolute magnitudes) of the test model electrical supply power look consistent with the experimental data for small longitudinal separation distances of the test model with respect to the small-scale building, i.e. $\mathrm{x} / \mathrm{R} \leq 3.84$. 


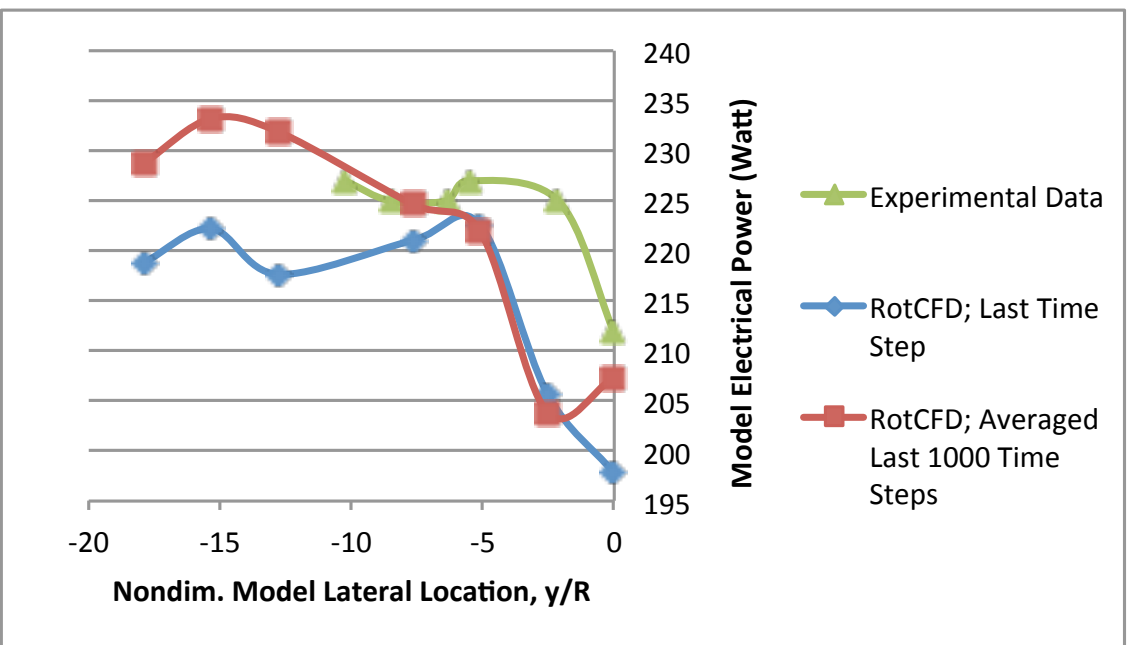

(a)

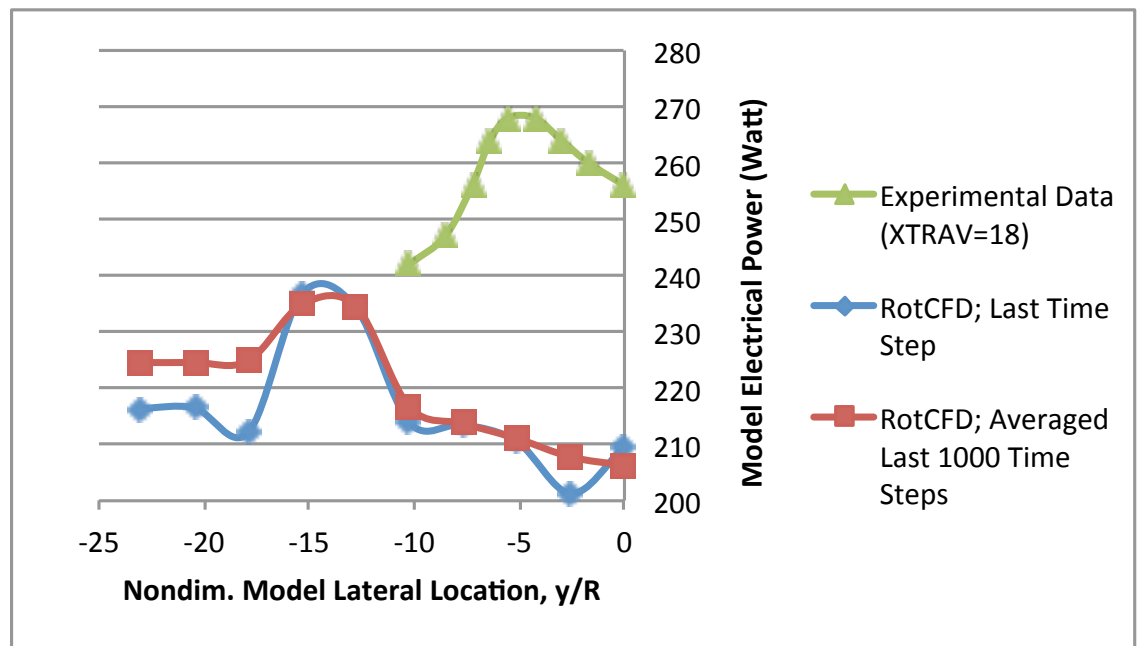

(b)
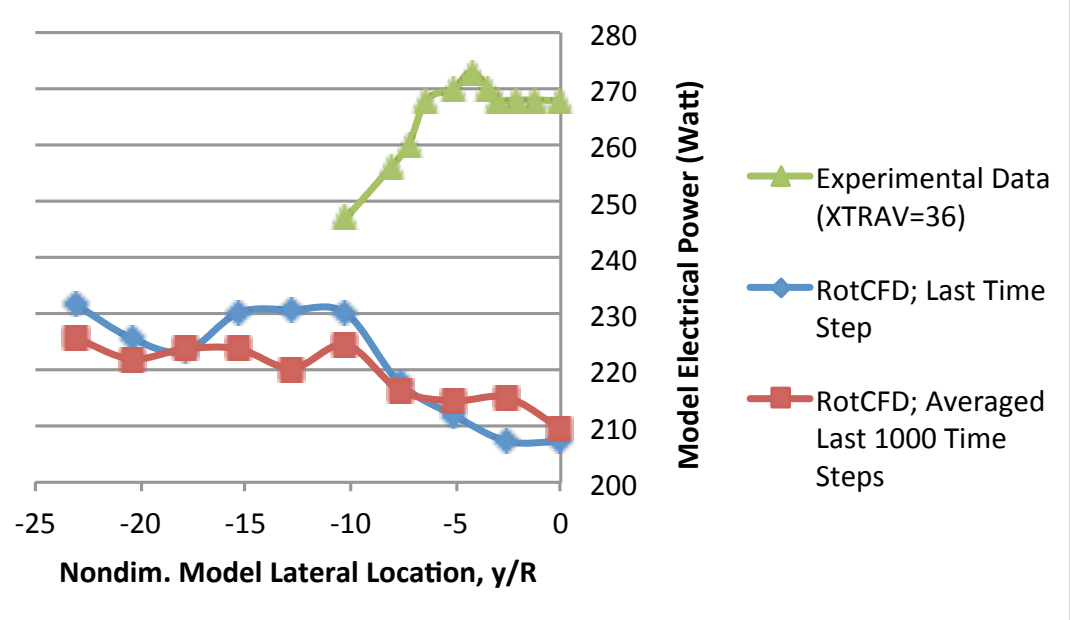

(c)

Figure 13a-c. Model electrical power correlation against experimental data: (a) $x / R=0 ;$ (b) $x / R=3.84$; (c) $\mathbf{x} / \mathbf{R}=\mathbf{7 . 6 7}$ 
For larger longitudinal separation distances, the predicted versus measured electrical power trends are inconsistent even in a general trend sense. This may be due to two factors. First, the RotCFD predictions were ran as fully turbulent cases, with the "realizable $\kappa-\varepsilon$ " turbulence model (Ref. 8). It may be that these cases need to be as laminar flow cases. That is left as future work, though. Second, the three-dimensional bluff body wake of the simulated building may be difficult to accurately predict, particularly as the tiltrotor model may be quite close to the upper boundary of the wake, in a region of intermittent flow.

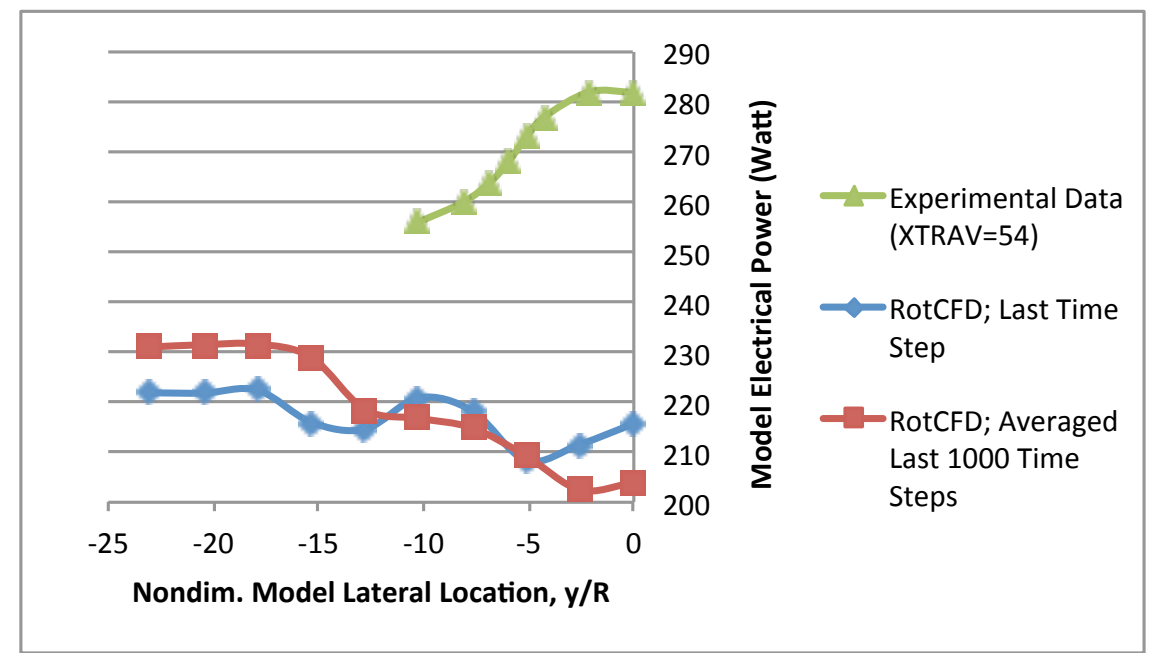

(d)

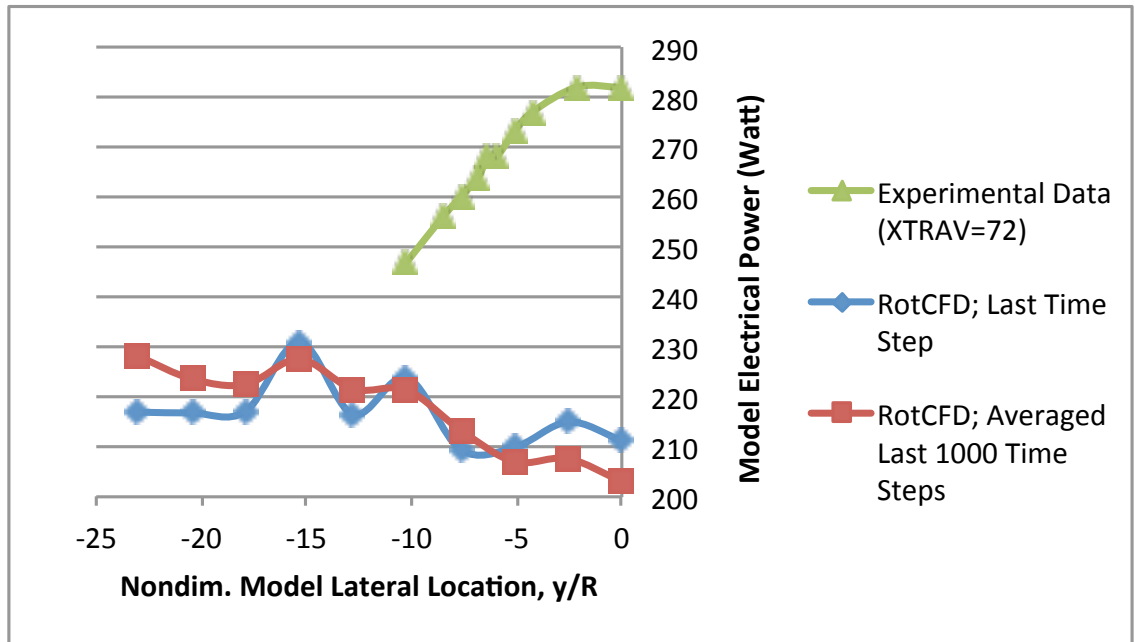

(e)

Figure 13d-e (Cont.). Model electrical power correlation against experimental data: (d) $x / R=11.5$ and (e) $\mathbf{x} / \mathbf{R}=\mathbf{1 5 . 3}$.

Figure 14 summarizes the comparison of predicted versus experimental measurements of Ref. 3 test model side force. There appears to be general agreement with respect to side force trends between the predicted and experimental results. 


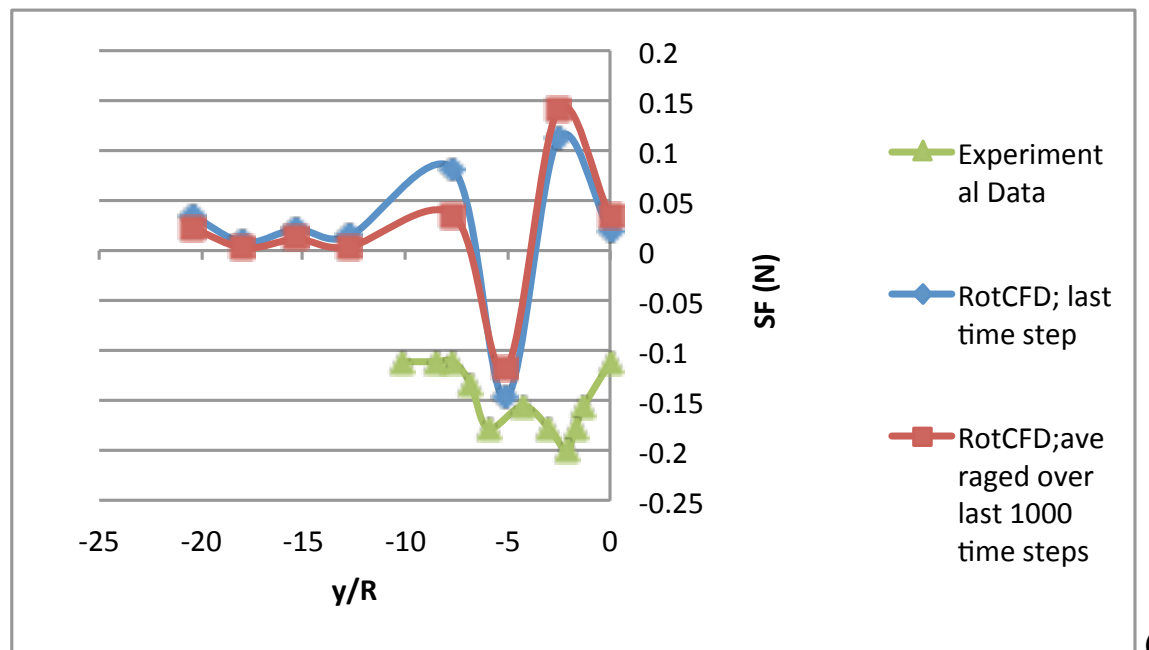

(a)

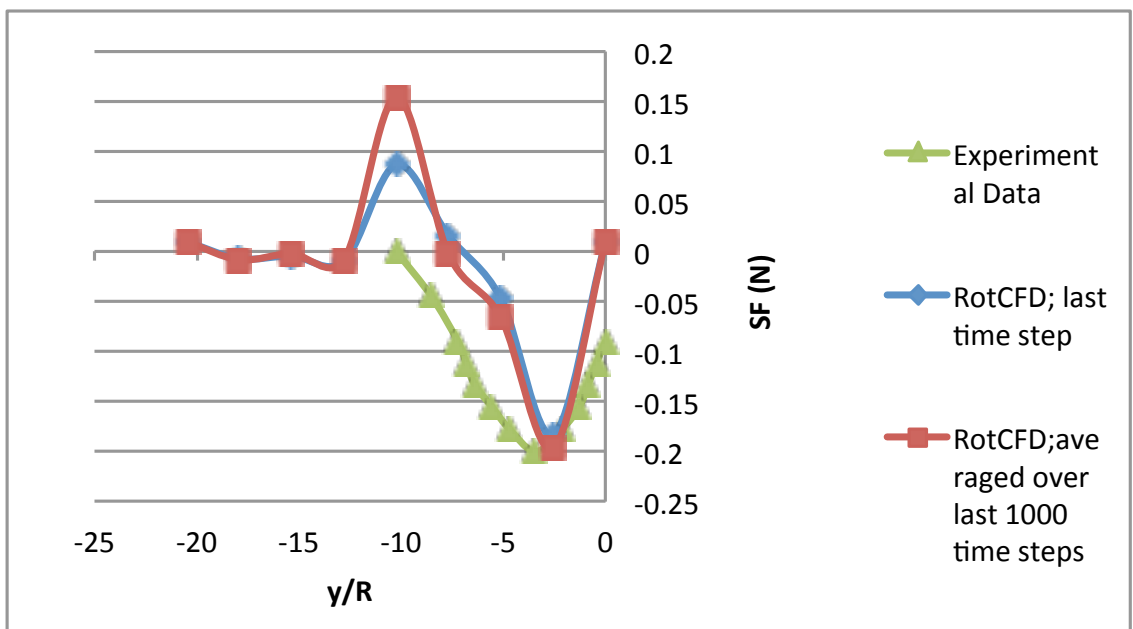

(b)

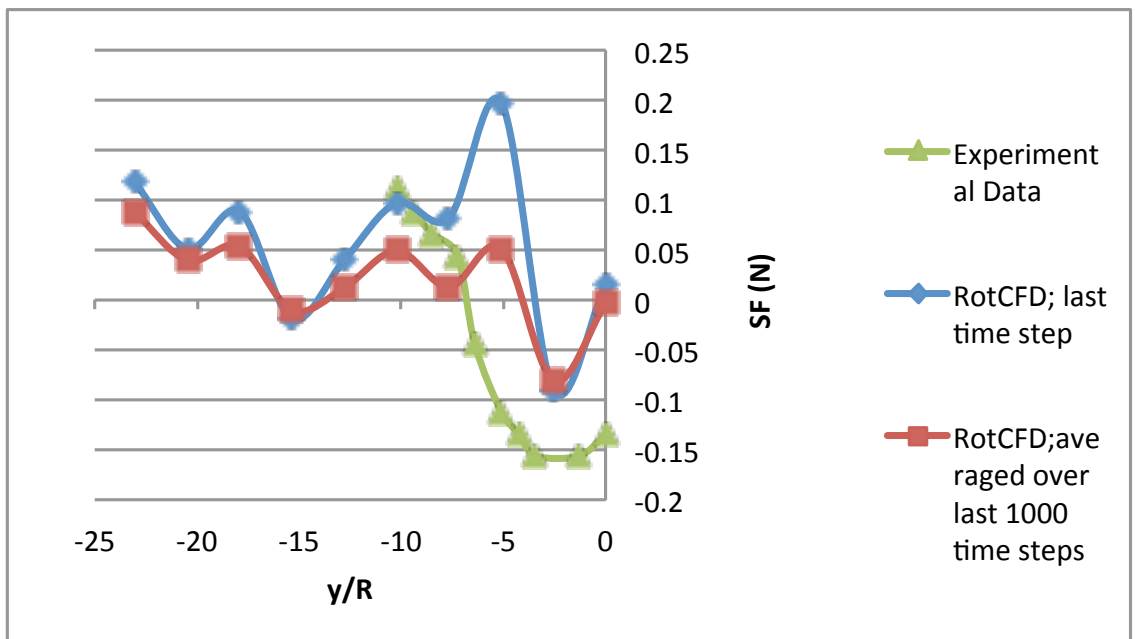

(c)

Figure 14a-c. Model side force correlation against experimental data: (a) $x / R=0 ;$ (b) $x / R=3.84 ;$ (c) $\mathbf{x} / \mathbf{R}=\mathbf{7 . 6 7}$ 


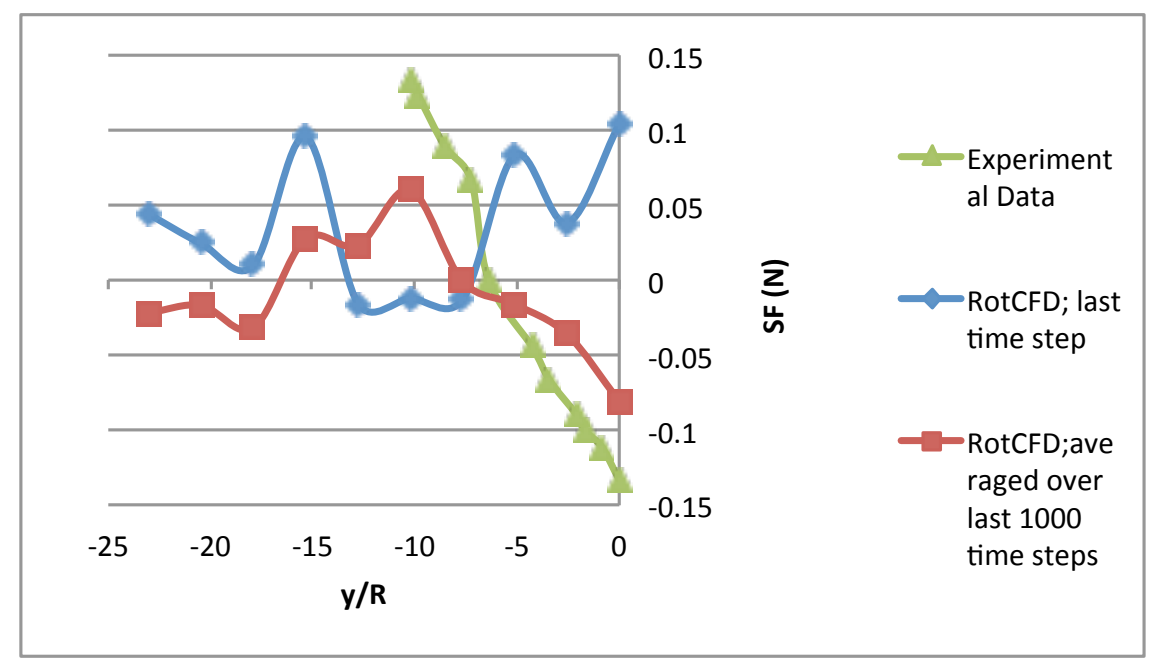

(d)

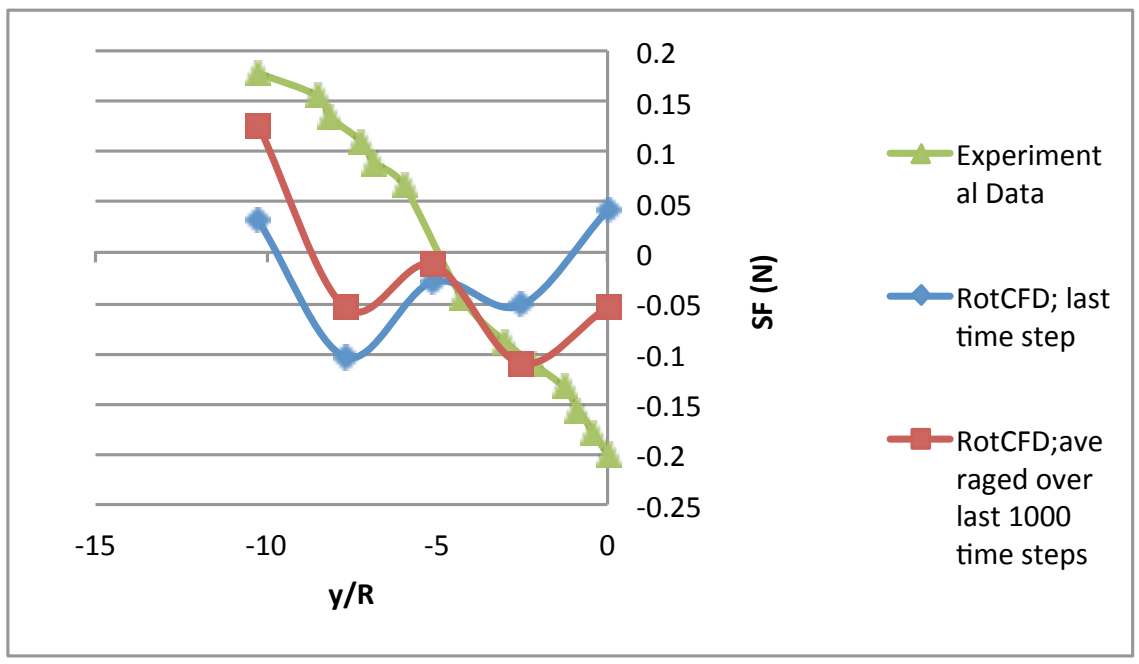

(e)

Figure 14d-e. Model side force correlation against experimental data: (d) $x / R=$ and (e) $x / R=$

Figures 15-16 summarize the test model moments. Figure 15 presents results for the combined rotors and fuselage forces and moment contribution and Fig. 16 is the rotors-only contribution to overall model yaw, pitch, and rolling moments. In both cases, the results are not that good. However, the rotors-only contribution results are consistently in better agreement with the Ref. 3 test results. RotCFD consistently predicts significant fuselage moment contributions that do not appear to manifest themselves in the Ref. 3 data. There are a number of potential reasons for this disagreement but one fundamental question that has to be left unanswered until future test results are acquired is whether or not the Ref. 3 test model configuration geometry compromises (as detailed in Ref. 2 and 9) with respect to the fuselage and empennage might be partly at fault for the disagreement in Fig. 15. But another partial explanation may lie in the fact that for computational fluid dynamic predictions of bluff body flow - as clearly the simulated building flow is - tend to demonstrate significant asymmetries in their downstream wakes. The RotCFD predictions clearly demonstrate such asymmetries (the building wake preferentially seeming to laterally shift to the left (looking down upon the building and the tiltrotor test model. Such a large wake asymmetry would reflect a commensurate shift in the force and moment trends of the $1 / 48^{\text {th }}$-scale V22 model (with respect to a model lateral displacement sweep/survey, as presented). 


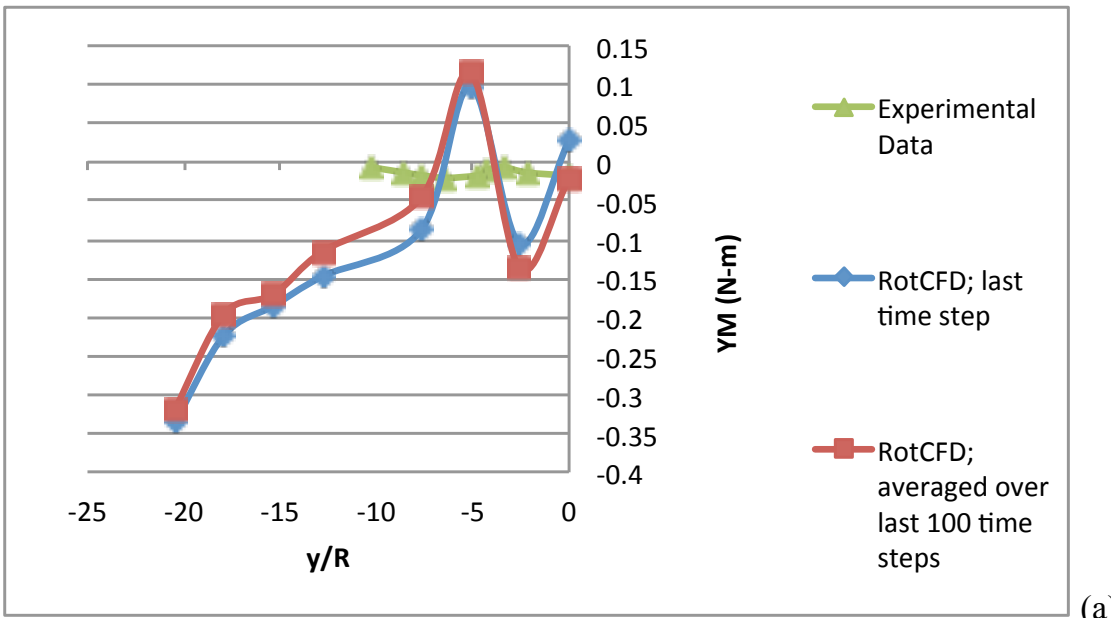

(a)

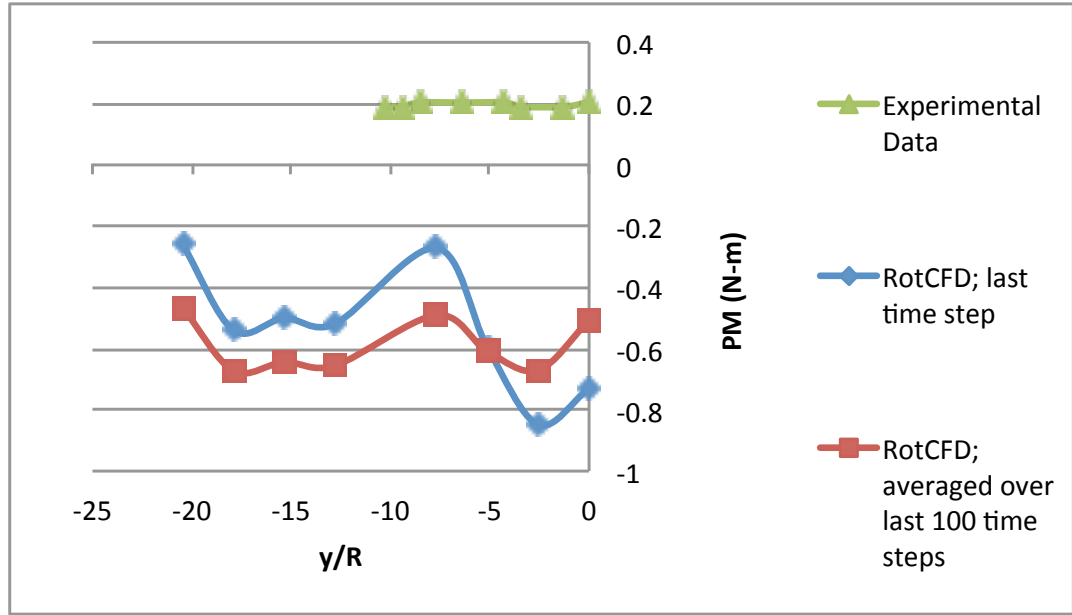

(b)

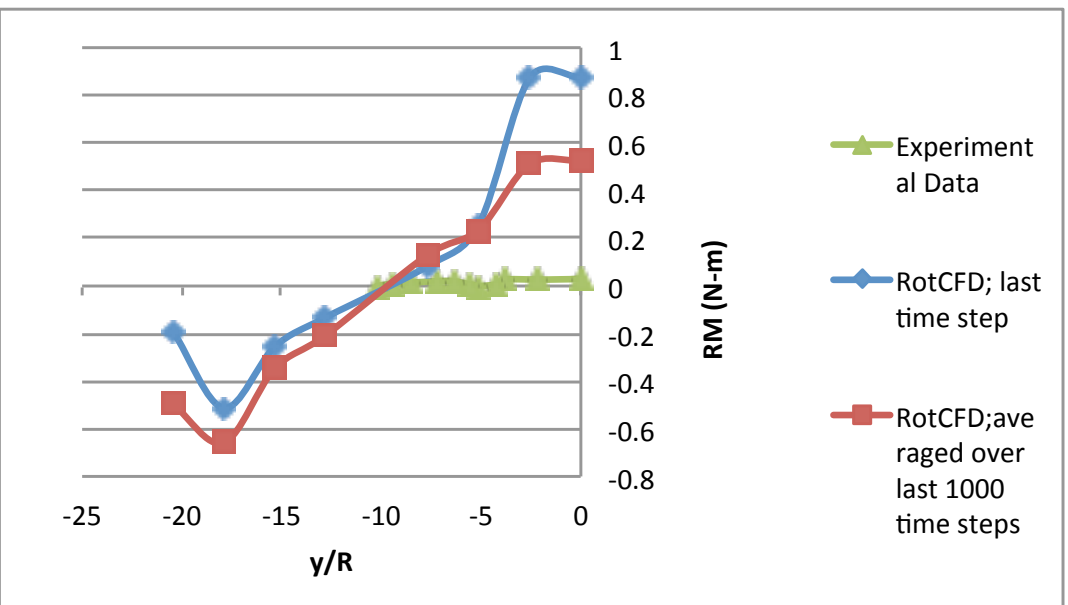

(c)

Figure 15a-c. RotCFD (combined rotors and fuselage forces and moments) comparison with experimental data ( $\mathrm{x} / \mathrm{R}=\mathbf{0})$ : (a) Yawing Moment ; (b) Pitching Moment ; (c) Rolling Moment 

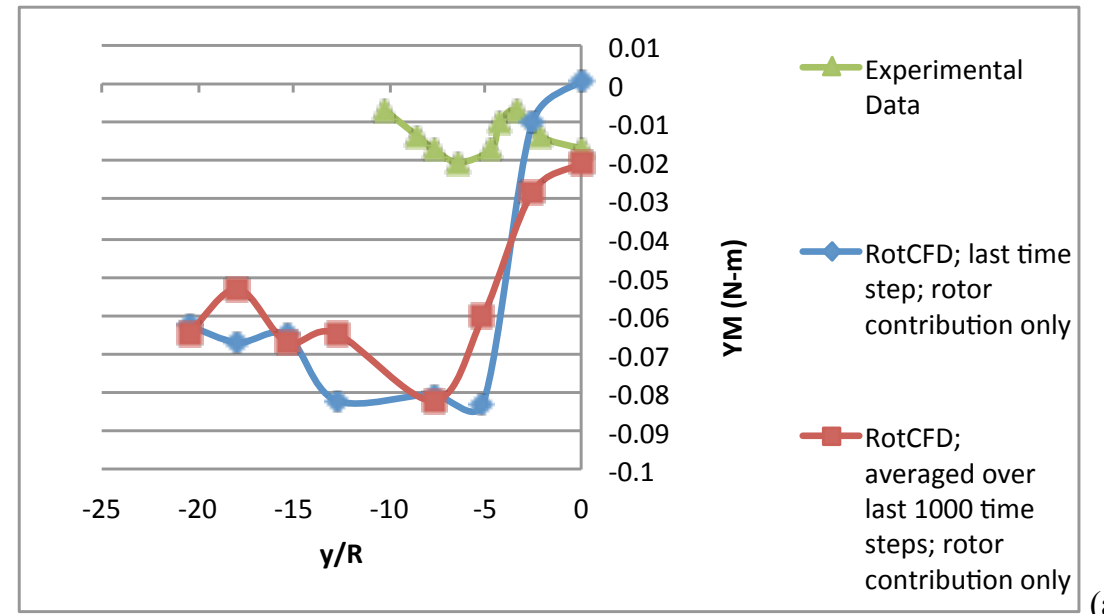

(a)
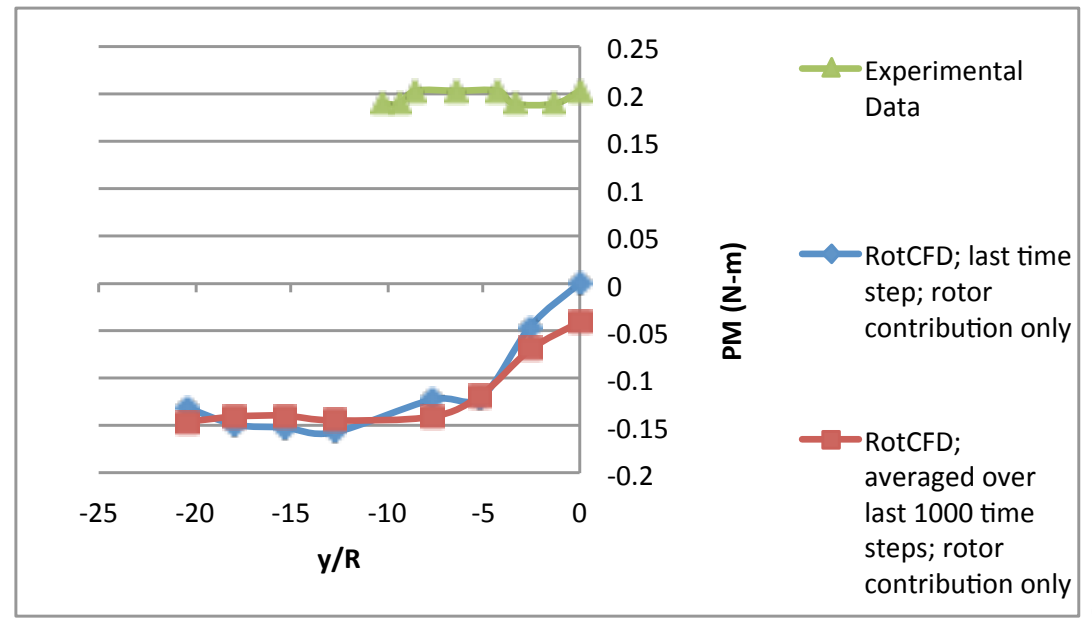

(b)

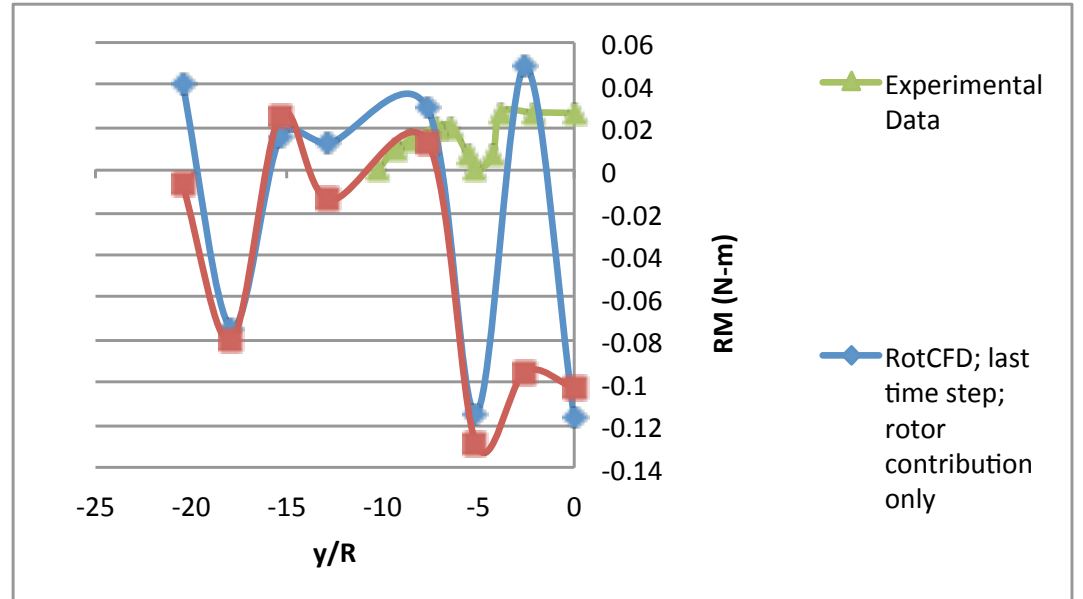

(c)

Figure 16a-c. RotCFD (rotors-only contribution forces and moments) comparison with experimental data ( $\mathrm{x} / \mathrm{R}=0$ ): (a) Yawing Moment ; (b) Pitching Moment ; (c) Rolling Moment 
Figure 17 illustrates flow visualization results for $\mathrm{x} / \mathrm{R}=11.51$ for the upper horizontal survey plane. Each flow visualization image is for the last time step of the solution, or time step $=2000$ and, therefore, $t=20$ seconds.
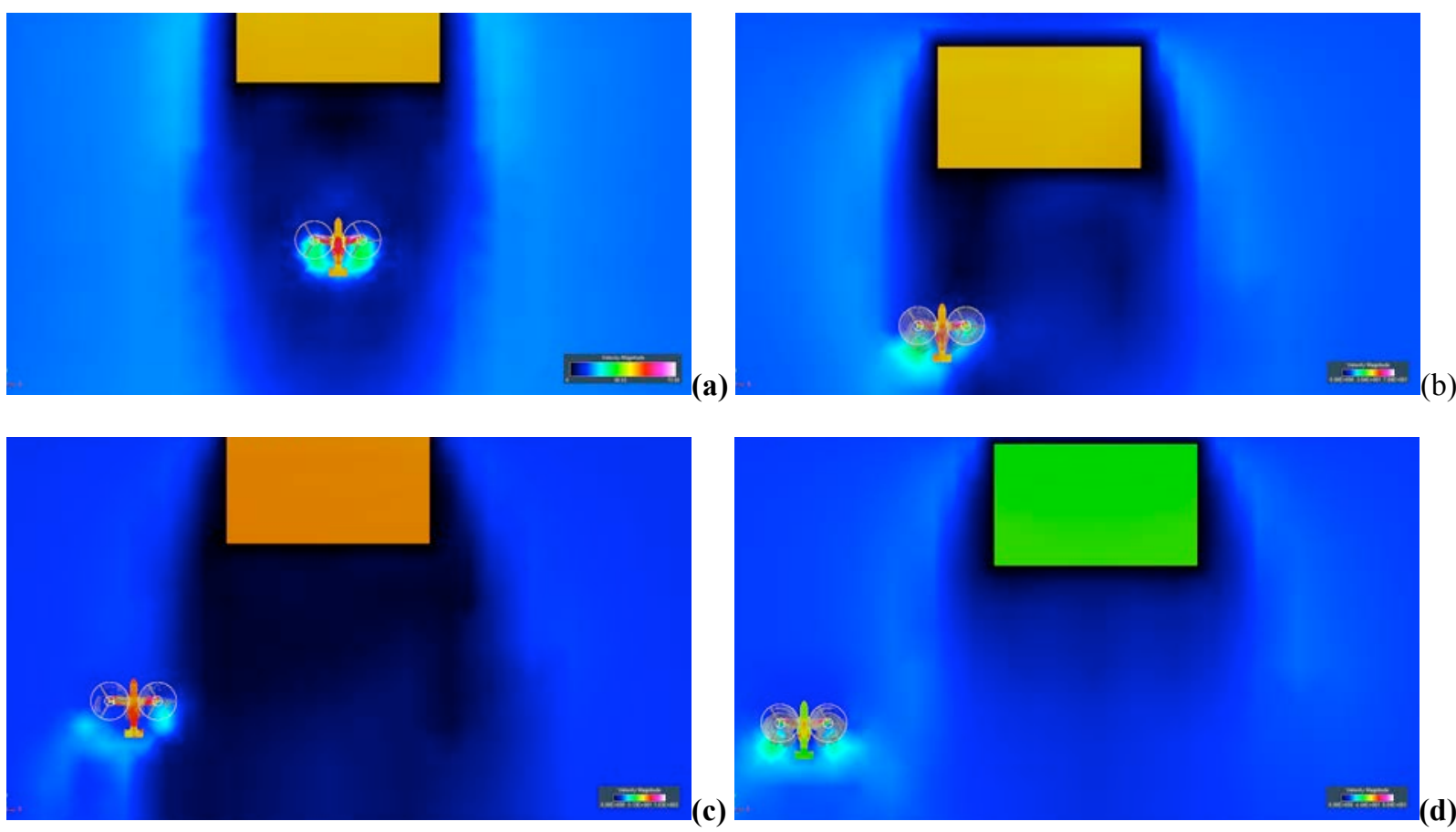

Figure 17. Prediction of upper surface pressure distribution (surface pressure scaling not held constant) of $1 / 48^{\text {th }}$ scale model with lateral displacement from the simulated building $(x / R=11.51):$ (a) $y / R=0 ;(b) y / R=-$ $5.11 ;$ (c) $y / R=-10.23 ;$ (d) $y / R=-15.35$

\section{Middle Survey Plane $(\mathrm{h} / \mathrm{R}=3.3)$}

Limited experimental data and CFD results are now presented for a horizontal survey plane through the middle of the simulated building at a height-to-radius ratio of $h / R=3.3$ from the base of building, on the ground. Figure 18 is a representative RotCFD flow visualization result for the one-forty-eighth-scale V-22 tiltrotor model in (very) close proximity to the building at $\mathrm{h} / \mathrm{R}=3.3$. Some of the fuselage surface pressure distribution asymmetry due to tiltrotor aircraft/building wake interactions, when both are in close proximity to each other, can be clearly in this upper surface planform view. This surface pressure distribution asymmetry, coupled with differences in forces and moments between the two rotors (one of which is for the most part closer to the building than the other), results in a significant variation in overall test model/vehicle forces and moments with lateral displacement of the test model with respect to the building. Longitudinal (downstream) displacements of the test model results in variation of overall test model forces and moments which is dependent upon the spatial-temporal distribution/evolution of the building's bluff body wake.

Figures 19 and 20 compare RotCFD results against the experimental data for the yawing, rolling, and pitching moments for the $1 / 48^{\text {th }}$-scale V-22 test model. 


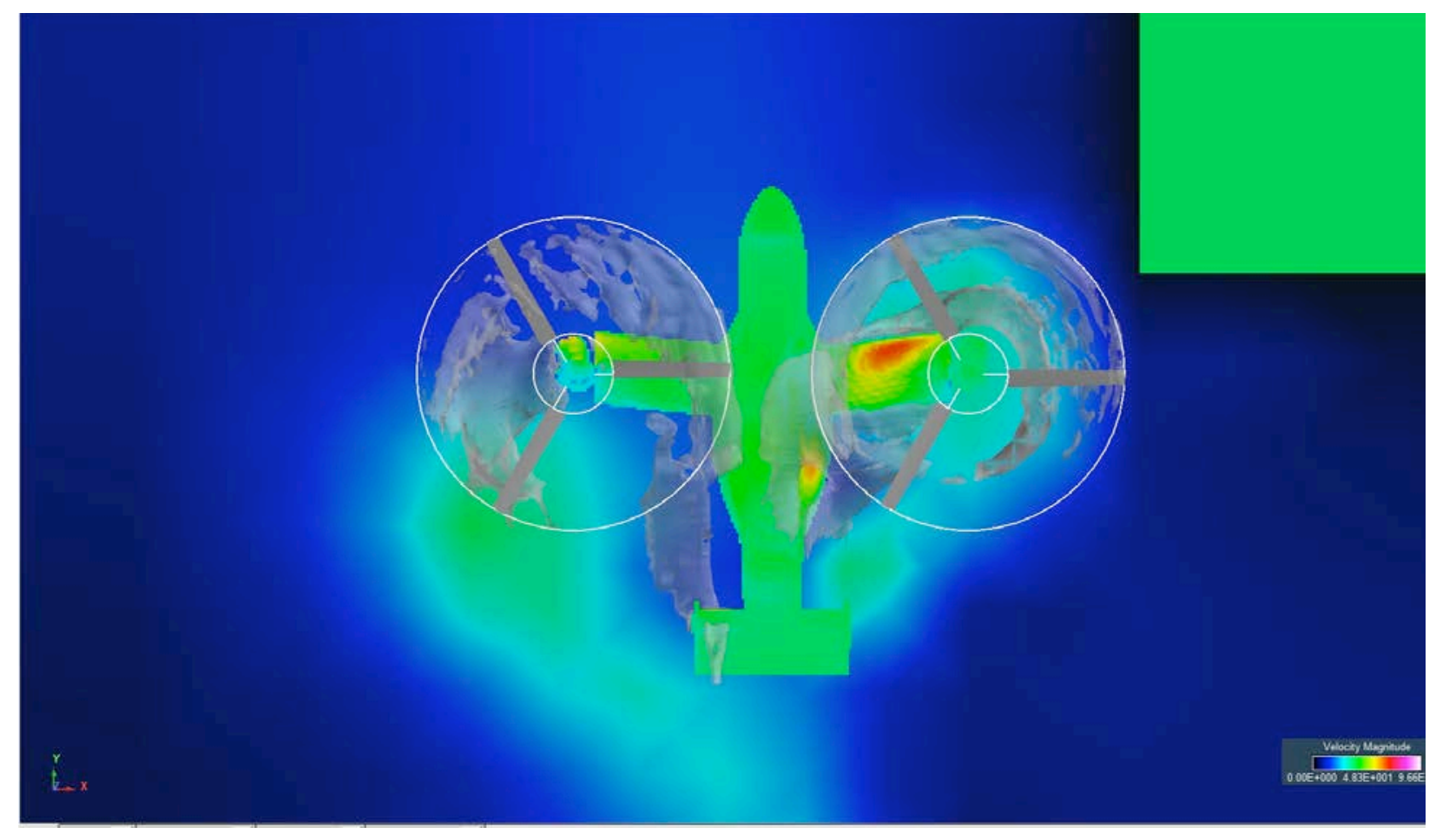

Figure 18. Representative RotCFD Flow Visualization Result for the tiltrotor model at $h / R=3.3$ (middle survey plane) 


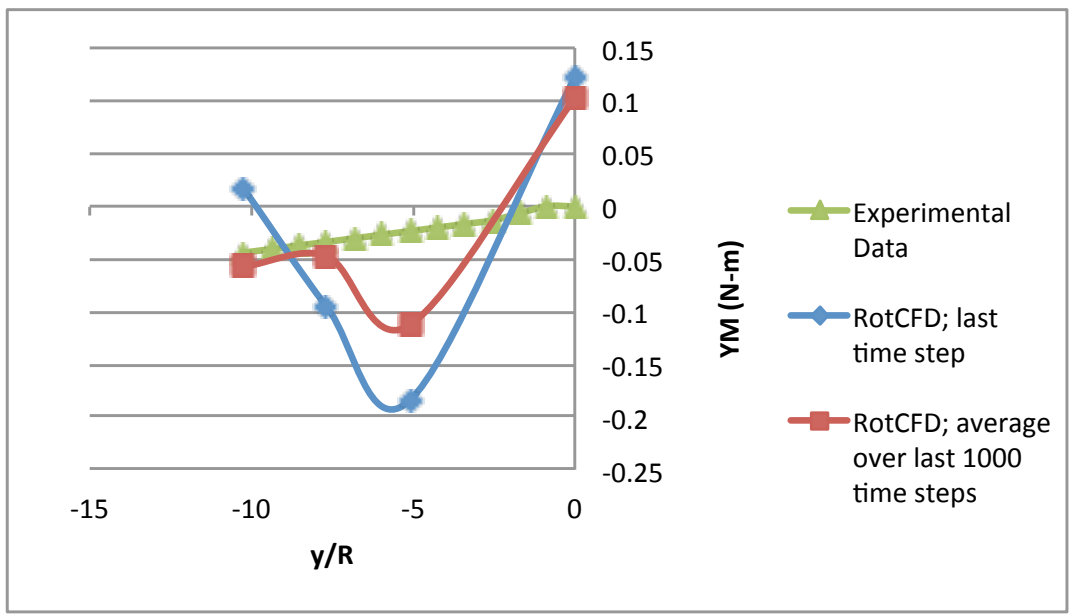

(a)

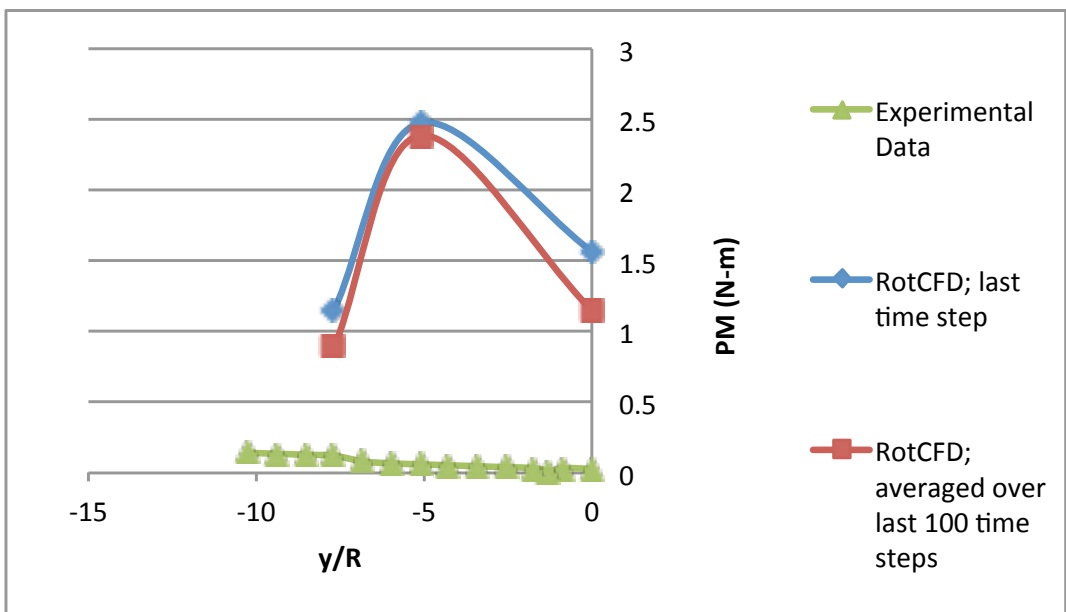

(b)

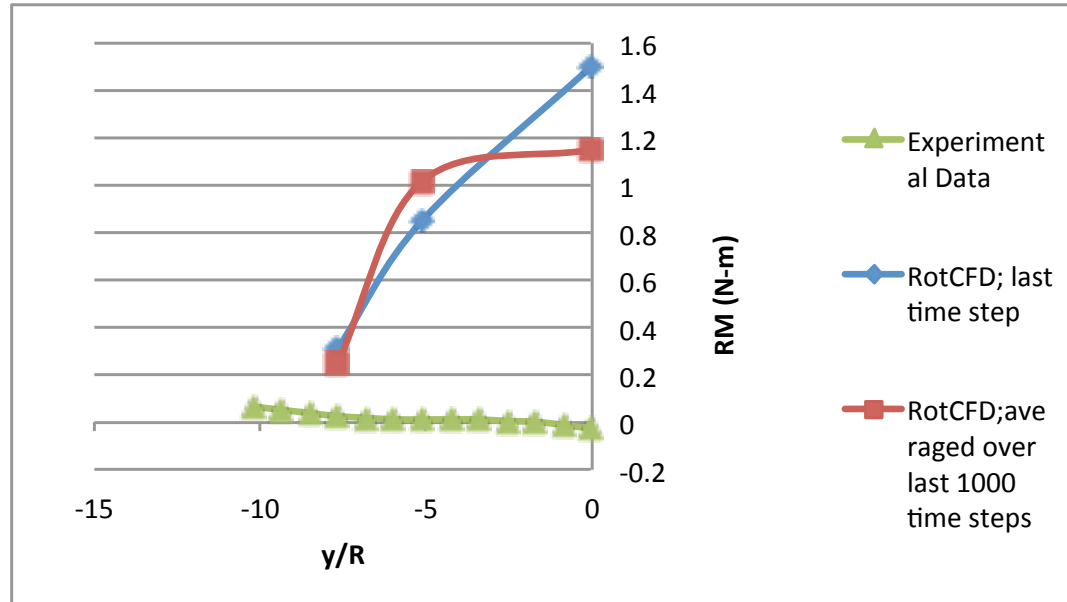

(c)

Figure 19a-c. RotCFD (combined rotors and fuselage forces and moments) comparison with experimental data ( $x / R=17.9)$ : (a) Yawing Moment ; (b) Pitching Moment ; (c) Rolling Moment 

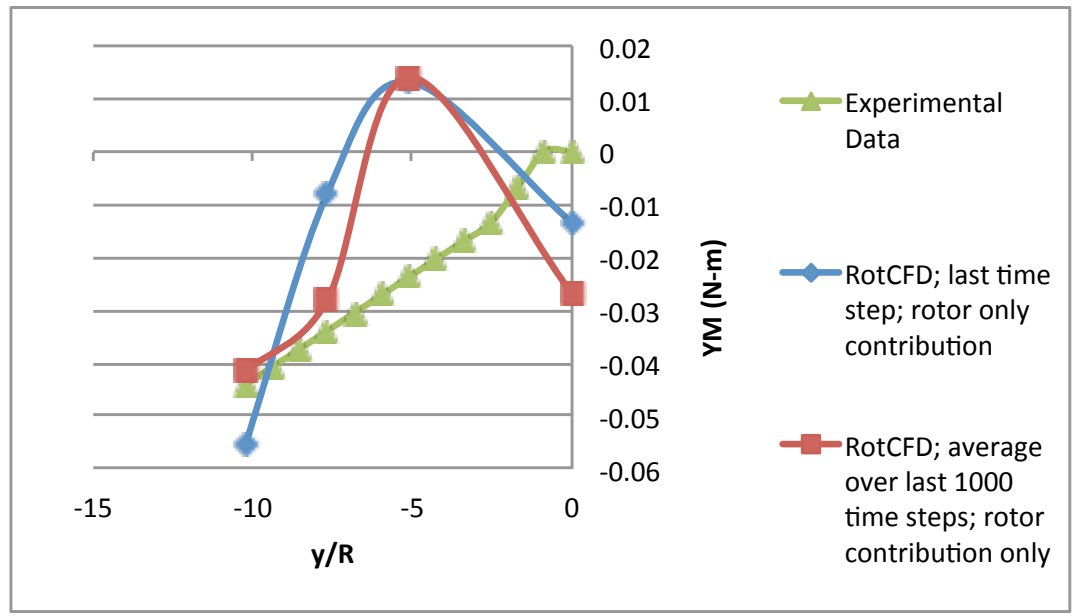

(a)

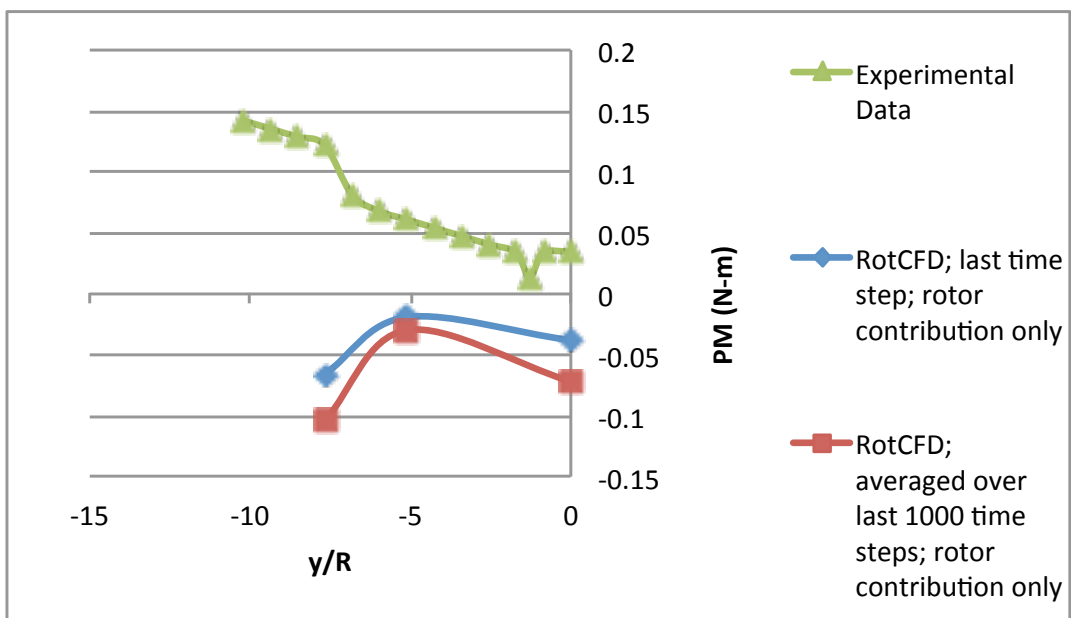

contribution only

(b)

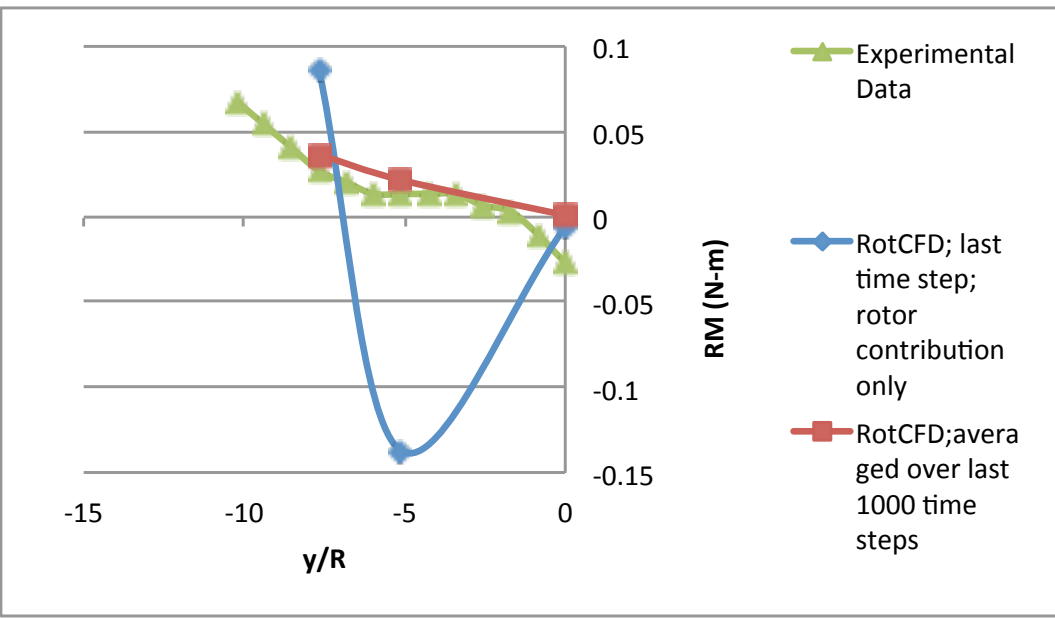

(c)

Figure 20a-c. RotCFD (rotors-only contribution forces and moments) comparison with experimental data (x/R=17.9): (a) Yawing Moment ; (b) Pitching Moment ; (c) Rolling Moment 
Figure 21 illustrates the wake interaction of the vehicle and building -- and the resulting transition in tiltrotor surface pressure distribution -- as the vehicle is laterally displaced away from the building at a $h / R=3.3$.
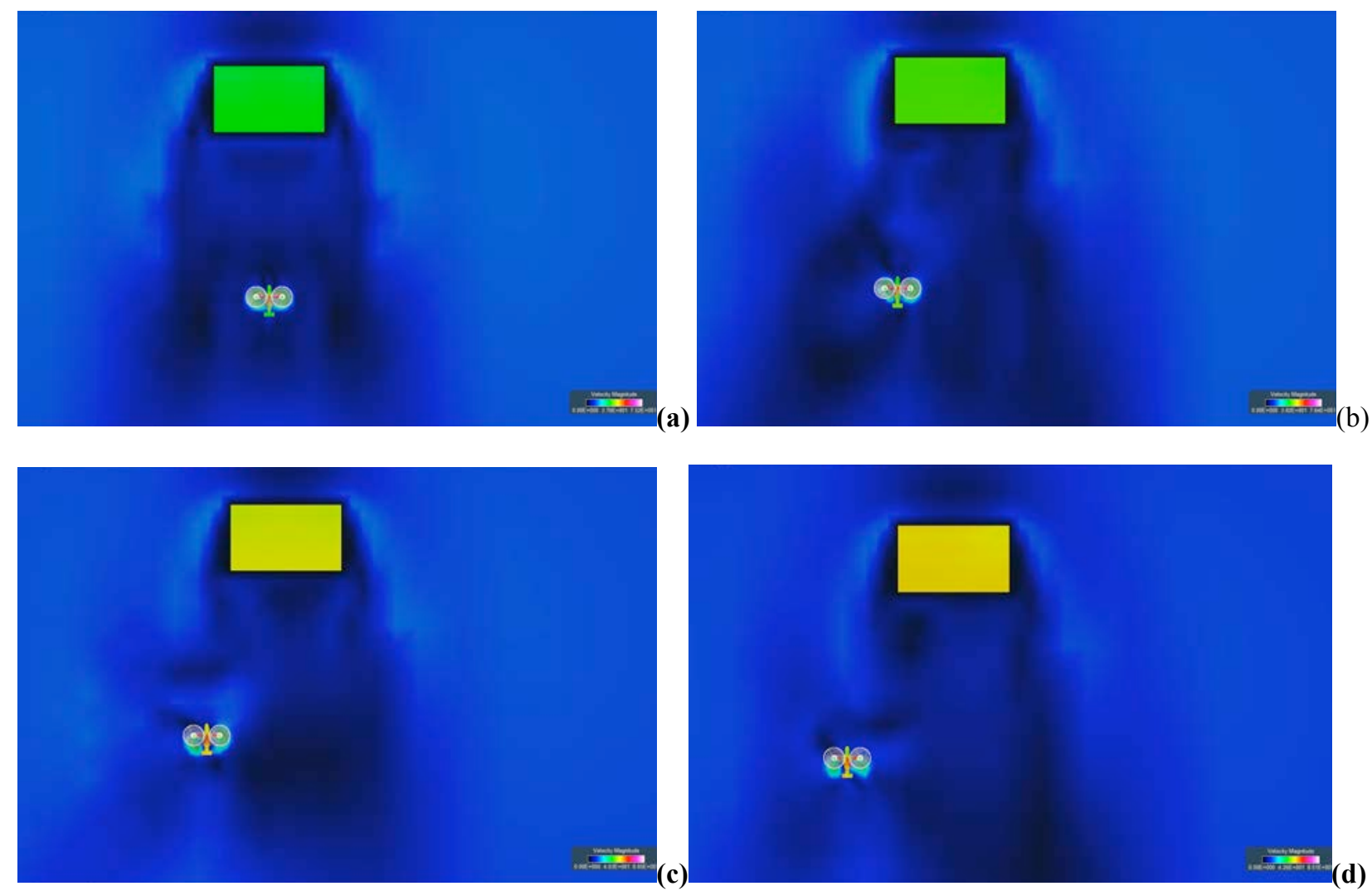

Figure 21. CFD predictions of upper surface pressure distribution of $1 / 48^{\text {th }}$ scale model as influence by lateral displacement from the simulated building ( $x / R=19.8$ ): (a) $y / R=0 ;$ (b) $y / R=-5.11 ;$ (c) $y / R=-7.67$; (d) $y / R=-10.23$

\section{Lower Survey Plane $(h / R=1.1)$}

Limited experimental data and CFD results are now presented for a horizontal survey plane near the bottom of the simulated building at a height-to-radius ratio of $h / R=1.1$ from the base of building, on the ground. Figure 22 is a representative RotCFD flow visualization result for the one-forty-eighth-scale V-22 tiltrotor model in close proximity to the building at $\mathrm{h} / \mathrm{R}=1.1$. AT this height-to-radius ratio the tiltrotor model is clearly in ground effect. Most of the results to follow will be further downstream (with respect to the simulated wind) of the building. 


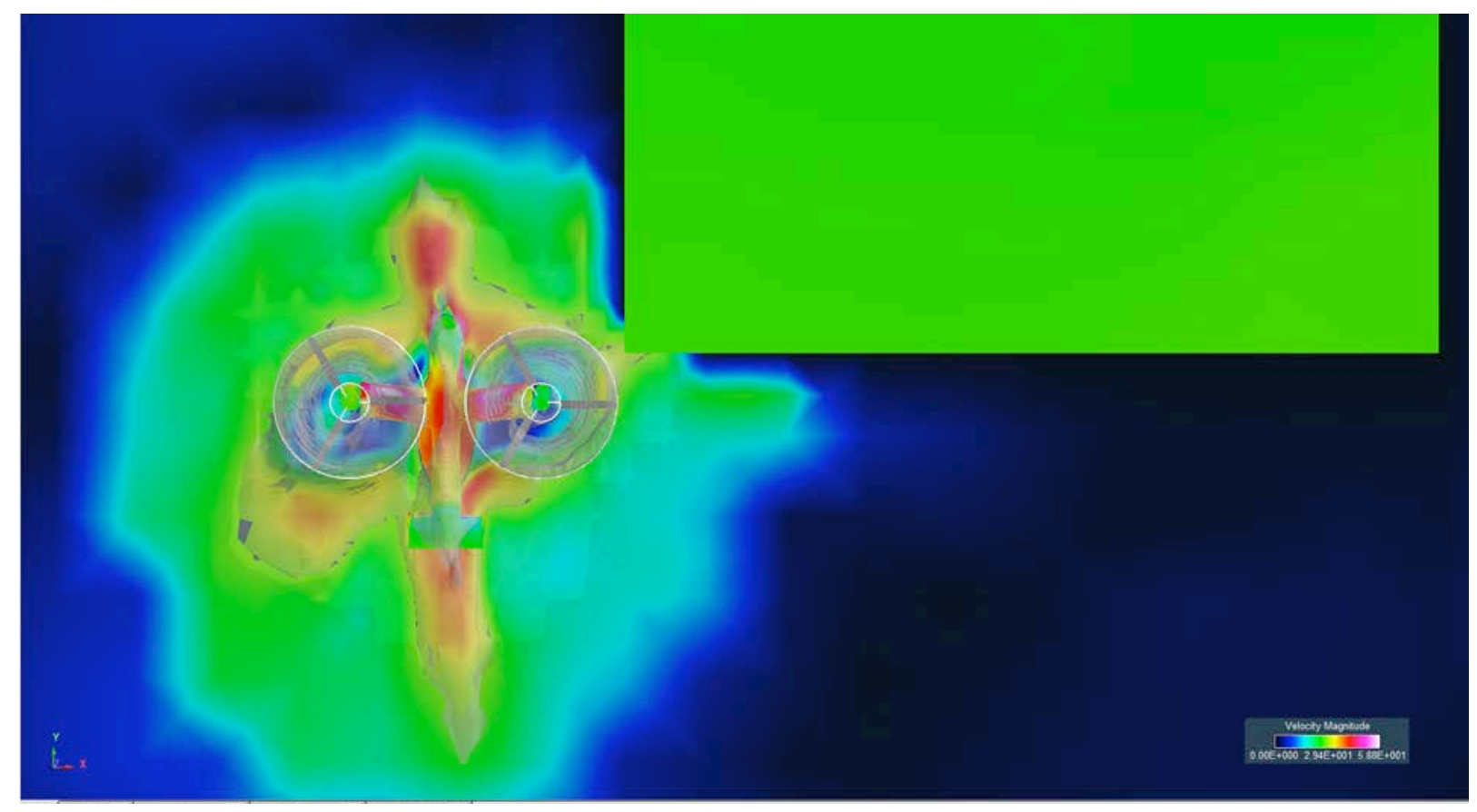

Figure 22. Representative RotCFD Flow Visualization Result for the tiltrotor model at $h / R=1.1$ (lower survey plane)

In general the RotCFD-predicted strong fore and aft regions of rotor outwash along the longitudinal axis of the vehicle are consistent with experimental observations and data for full-scale tiltrotor aircraft such as the V-22 and the XV-15 flight research aircraft when operating near the ground (Ref. 10). Other than this general observation it is beyond the scope of this paper to attempt a correlation between RotCFD and such experimental tiltrotor outwash data. However, such rotor outwash prediction work is of great interest and will be pursued in the future.

Figures 23 and 24 compare RotCFD results against the experimental data for the yawing, rolling, and pitching moments for the $1 / 48^{\text {th }}$-scale V-22 test model. 


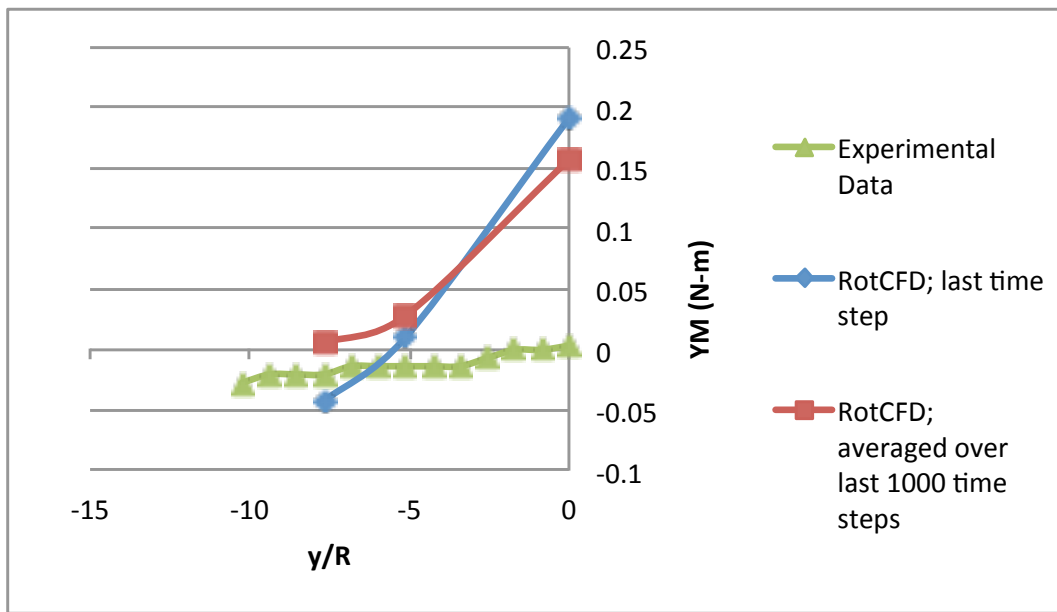

(a)

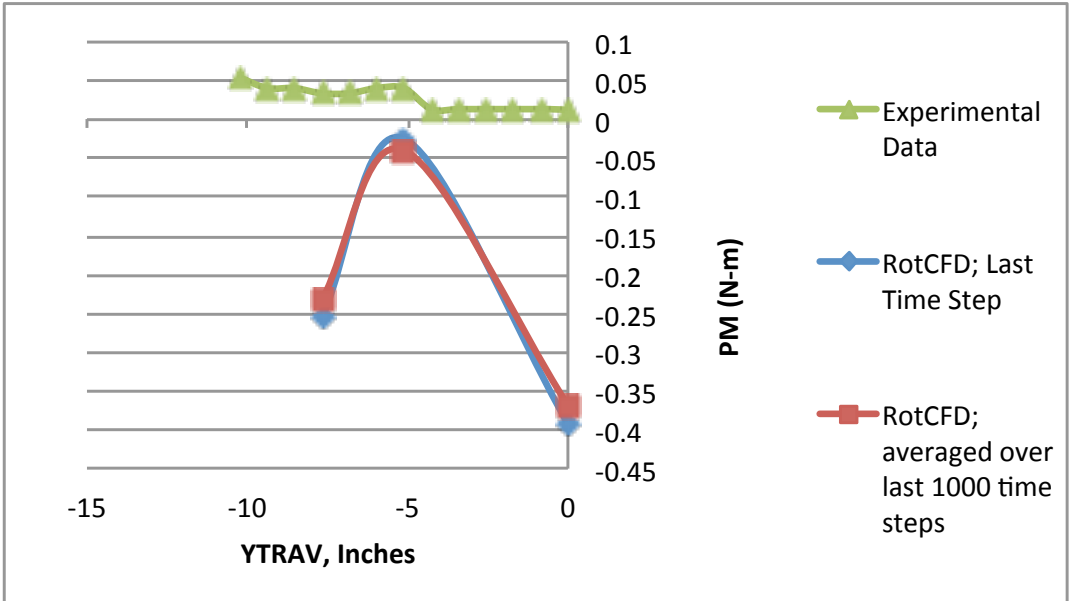

(b)

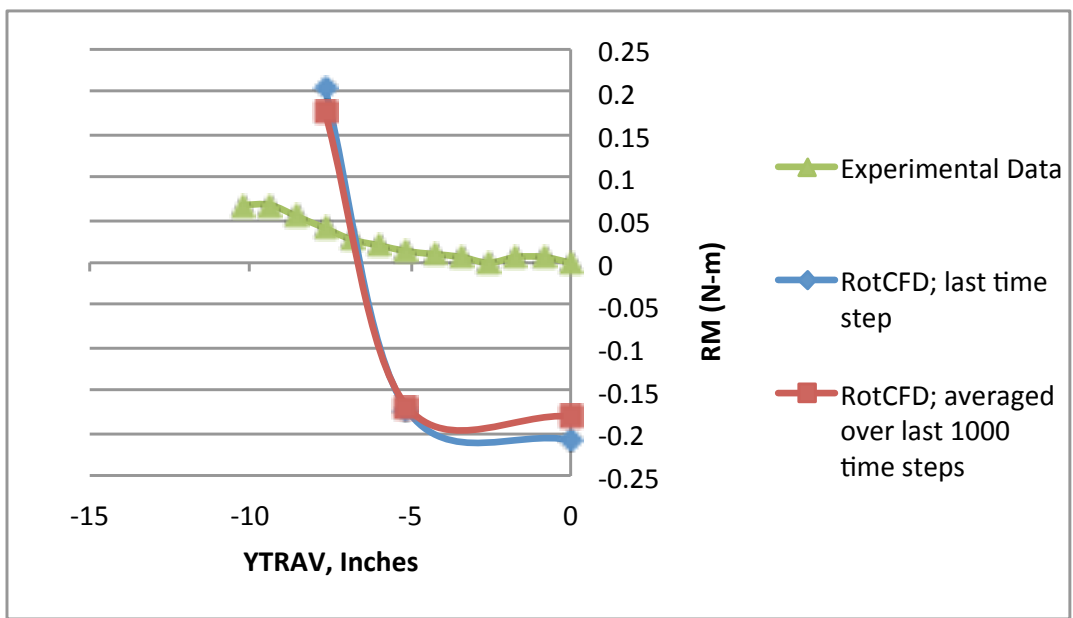

(c)

Figure 23a-c. RotCFD (combined rotors and fuselage forces and moments) comparison with experimental data ( $\mathrm{x} / \mathrm{R}=17.9)$ : (a) Yawing Moment ; (b) Pitching Moment ; (c) Rolling Moment 


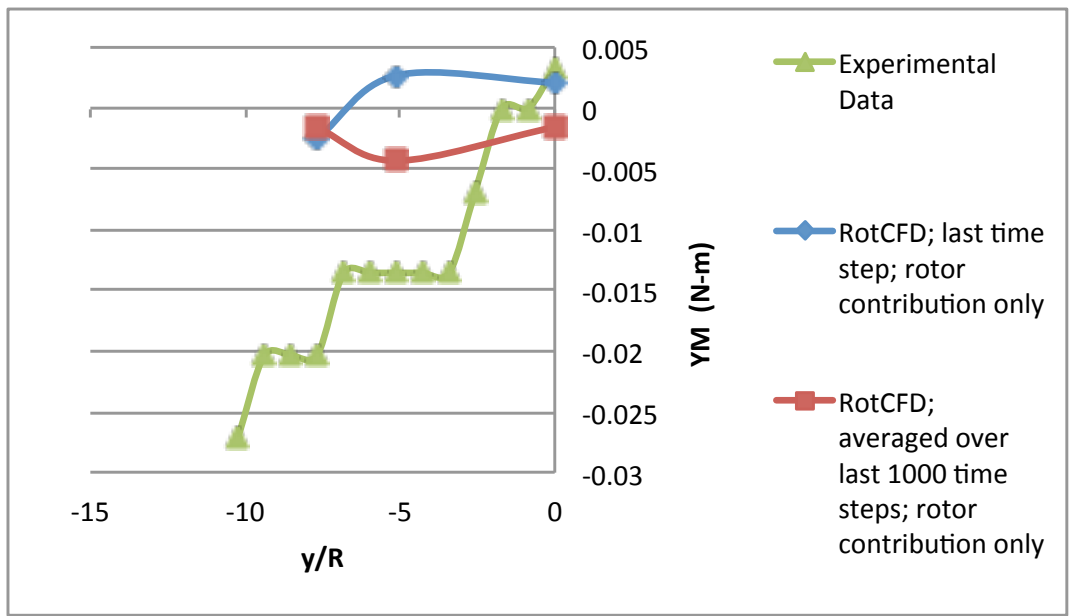

(a)
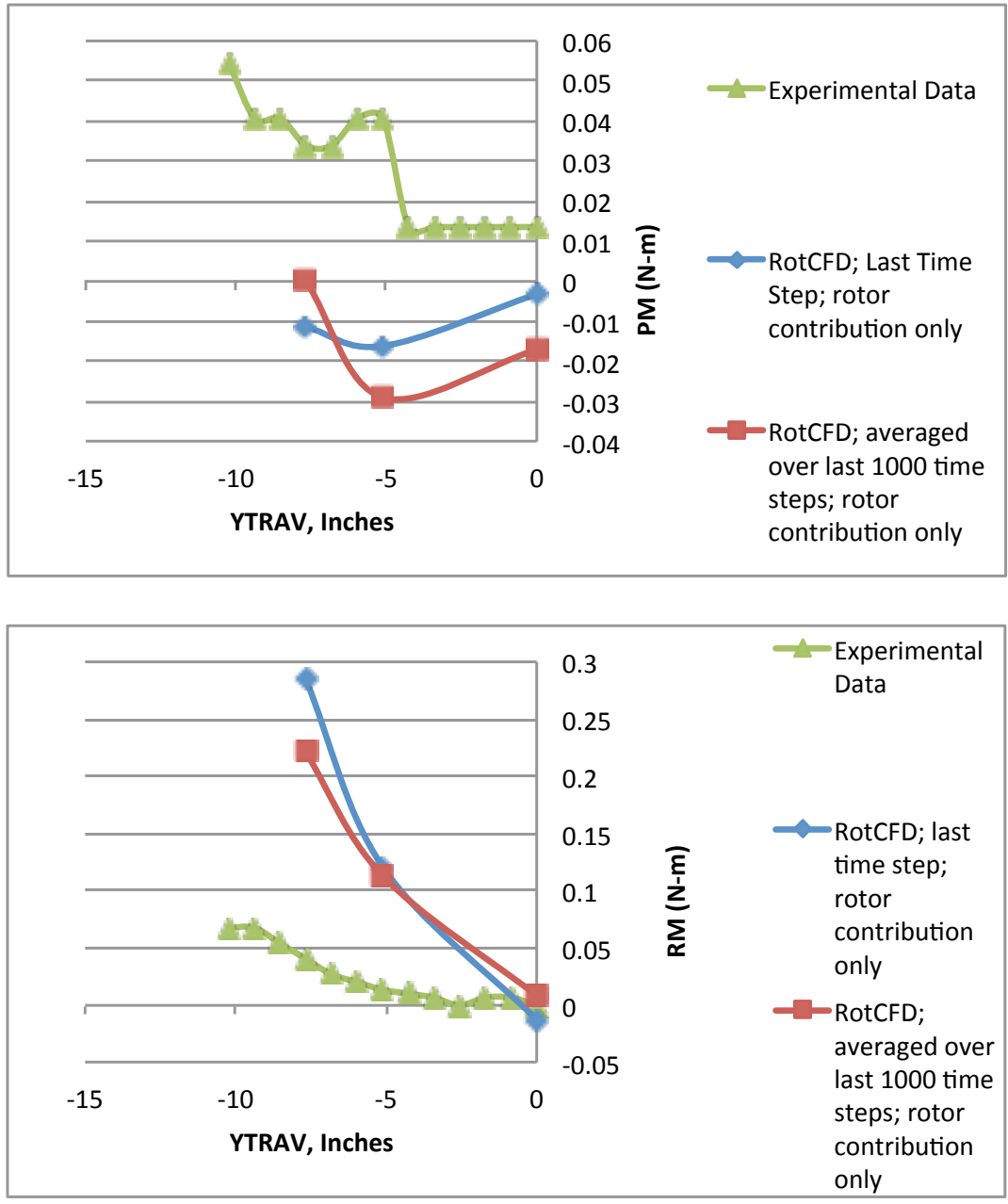

(c)

Figure 24a-c. RotCFD (rotors-only contribution forces and moments) comparison with experimental data (x/R=17.9): (a) Yawing Moment ; (b) Pitching Moment ; (c) Rolling Moment 
Figure 25 illustrates the wake interaction of the vehicle and building - and the resulting transition in tiltrotor surface pressure distribution - as the vehicle is laterally displaced away from the building at a $h / R=1.1$. As can be seen in this figure there are also subtle shifts in the rotor outwash of the vehicle near the ground as a consequence of the vehicle traversing into and out of the building wake.
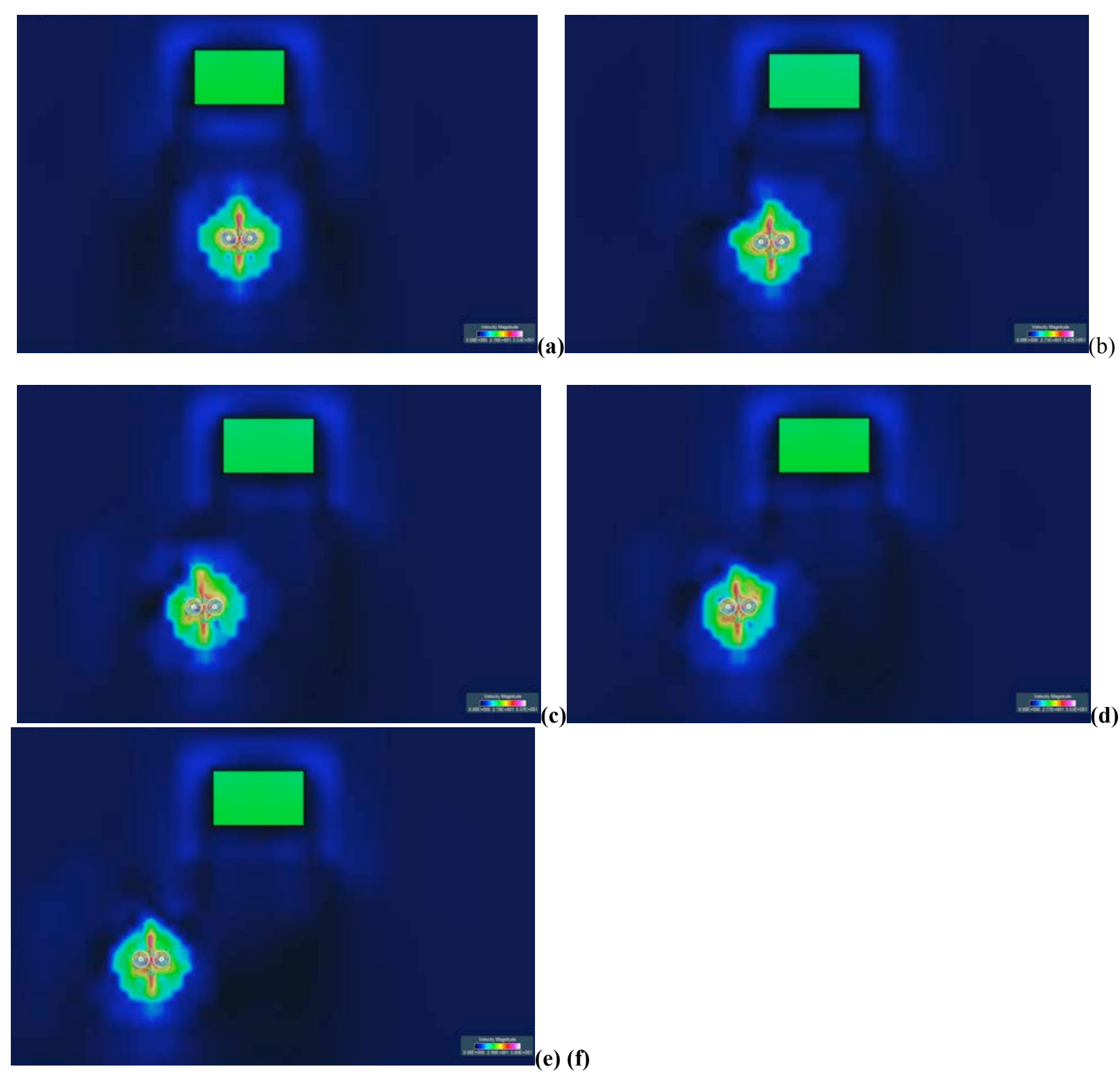

Figure 25. CFD predictions of upper surface pressure distribution of $1 / 48^{\text {th }}$ scale model as influence by lateral displacement from the simulated building $(\mathrm{x} / \mathrm{R}=19.8)$ : (a) $\mathrm{y} / \mathrm{R}=0$; (b) $\mathrm{y} / \mathrm{R}=-5.11$; (c) $\mathrm{y} / \mathrm{R}=-7.67$; (d) $\mathrm{y} / \mathrm{R}=-10.23$; $\mathbf{y} / \mathbf{R}=-12.79 ; y / R=-15.25$ 


\section{Future Work}

The ultimate outcome of this study is anticipated to be threefold. First, the information gleaned from this study will help refine future NASA reference designs for small- to moderate-sized civil tiltrotor aircraft. Second, the results from this study might help influence future vertiport design considerations, particularly those that may be sited on top of urban buildings or in the midst of an urban canyon (amongst the buildings, on the ground); Fig. 26. Third, the results might also influence the concepts of operation of such vehicles in the urban/city-center environment such that the efficiency and safety of CTR operations at urban vertiports can be successfully balanced.

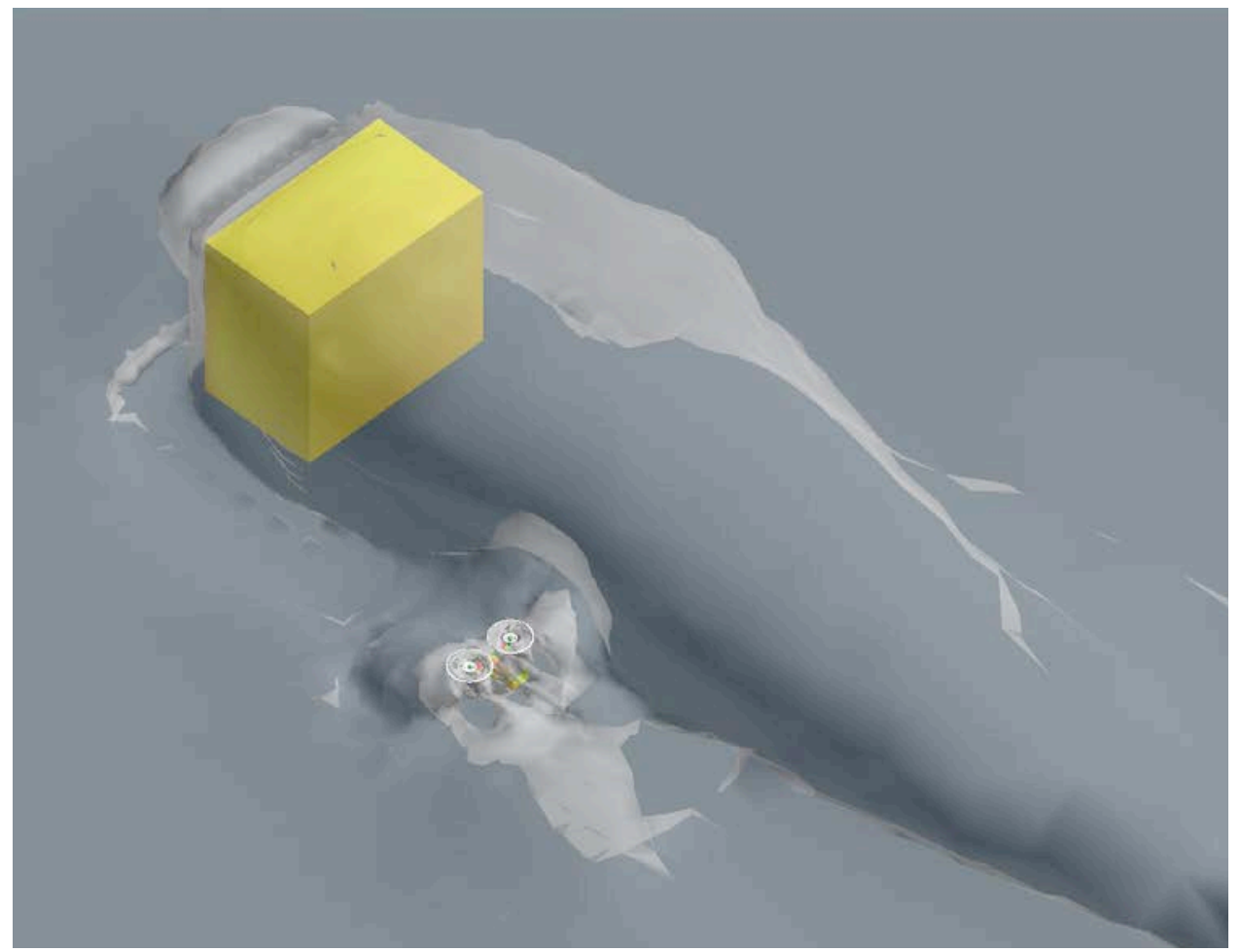

Figure 26. Civil tiltrotor aircraft operating in and out of center-center vertiports (isosurface visualization of vortex magnitude for building and tiltorotor wakes

\section{Concluding Remarks}

A mid-fidelity computational fluid dynamics tool, one especially tailored for the study of rotary-wing vehicles, is being applied to the problem of rotor/wake interactions in the urban vertiport operating environment. The computational fluid dynamics results are validated against experimental data from previous wind tunnel test campaigns. The correlation results for in-ground-effect results for the Ref. 2 wind tunnel test showed reasonably good agreement but required accurate prediction of both rotor thrust performance and well as test model vertical body forces. The correlation results for the building/tiltrotor aircraft wake interaction study of Ref. 3 was mixed. The $1 / 48^{\text {th }}$-scale V22 forces and moments (particularly moments) were much larger in magnitude than the measured 
test model loads; in a number of cases the test model trends with lateral displacement with respect to the simulated building were not even changing in the same direction as the measured moments. There are several reasons why this might be case including: (1) use of a fully turbulent CFD solution; (2) the use of a scaled V22 model for the CFD geometry versus the more simplistic model tested which included an exposed electric motor; (3) large unfaired cutouts on the upper fuselage surface; (4) and the interaction aerodynamics effects of a sting mount (which was not modeled); (5) a relatively coarse gridding for the building (though a fine grid for the test model); and (6) a predicted asymmetry of the building wake that could have biased the moment trends through a lateral displacement delta offset. More work is required in this important area of research so as to be able to be a future engineering aid for enabling tiltrotor and vertiport operational integration.

\section{Acknowledgments}

This work was supported in part by the Rotary Wing (RW) and Revolutionary Vertical Lift Technology (RVLT) projects of the NASA Aeronautics Research Mission Directorate. Thanks are extended to Drs. Preston Martin (U.S. Army) and Gloria Yamauchi (NASA) for their pioneering experimental investigations into rotorcraft wake interactions. Thanks are also extended to Prof. Ganesh Rajagopalan (Iowa State University) for his development and support of the RotCFD rotorcraft computational fluid dynamics software tool. Finally, this paper acknowledges the program leadership of Dr. John Coy (NASA Ret.) for VTOL aircraft or, rather, runway independent aircraft research.

\section{References}

${ }^{1}$ Young, L.A., Rajagopalan, G. and Yamauchi, G.K., "Simulated Rotor Wake Interactions Resulting from Civil Tiltrotor Aircraft Operations Near Vertiport Terminals," 51st AIAA Aerospace Sciences Meeting, AIAA 20130801, Grapevine, TX, January 7-10, 2013.

${ }^{2}$ Yamauchi, G. K., Wadcock, A. J. and Derby, M. R., "Measured Aerodynamic Interaction of Two Tiltrotors," Proceedings of the 59th Annual Forum of the American Helicopter Society, Phoenix, Arizona, May 6-8, 2003.

${ }^{3}$ Martin, P., "Tiltrotor/Building Interaction Test: 7x10 Wind Tunnel," Private Communication/Unpublished Data, May 2011.

${ }^{4}$ Hoerner, S.F., Fluid-Dynamic Drag, Self-published, 1965.

${ }^{5}$ Johnson, W., Yamauchi, G. K., Derby, M. R. and Wadcock, A. J., "Wind Tunnel Measurements and Calculations Of Aerodynamic Interactions Between Tiltrotor Aircraft," Proceedings of the 41st Aerospace Sciences Meeting and Exhibit, Reno, Nevada, January 6-9, 2003.

${ }^{6}$ Patankar, S.V., Numerical Heat Transfer and Fluid Flow, Hemisphere Publishing Corp, New York, 1980.

${ }^{7}$ Rajagopalan, G., "RotCFD a Tool for Aerodynamic Interference of Rotors: Validation and Capabilities," Future Vertical Lift Aircraft Design Conference, San Francisco, CA, January 18-20, 2012.

${ }^{8}$ Shih, T. H., Liou, W. W., Shabbir, A., Yang, Z. and Zhiu, J. (1995), “ A New Eddy-Viscosity Model for High Reynolds Number Turbulent Flows-Model Development and Validation," Computers and Fluids, 24(3), pp. 227238.

${ }^{9}$ Derby, M.R. and Yamauchi, G.K., "Design of $1 / 48^{\text {th }}$-Scale Models for Ship/Rotorcraft Interaction Studies," $21^{\text {st }}$ Applied Aerodynamics Conference, AIAA 2003-3952, Orlando, FL, June 23-26, 2003.

${ }^{10}$ Smith, R.D., Ed., "Heliport/Vertiport Design Deliberations, 1997-2000,” DOT/FAA/ND-00/1, May 2001. 


\section{Appendix}

This appendix encompasses a number of additional longitudinal $x / R$ survey stations for the upper $(z / R=2)$ horizontal survey plane. Additionally, comparisons are made between the Ref. 1 experimental data and the computational fluid dynamics results of RotCFD for scaled test model yaw, pitch, and roll moments.

Figure 27 illustrates the moment center location for the RotCFD predictions. The experimental data taken for both Ref. 1 and Ref. 2 are assumed to be at the described location noted in Ref. 2 and Ref. 13.
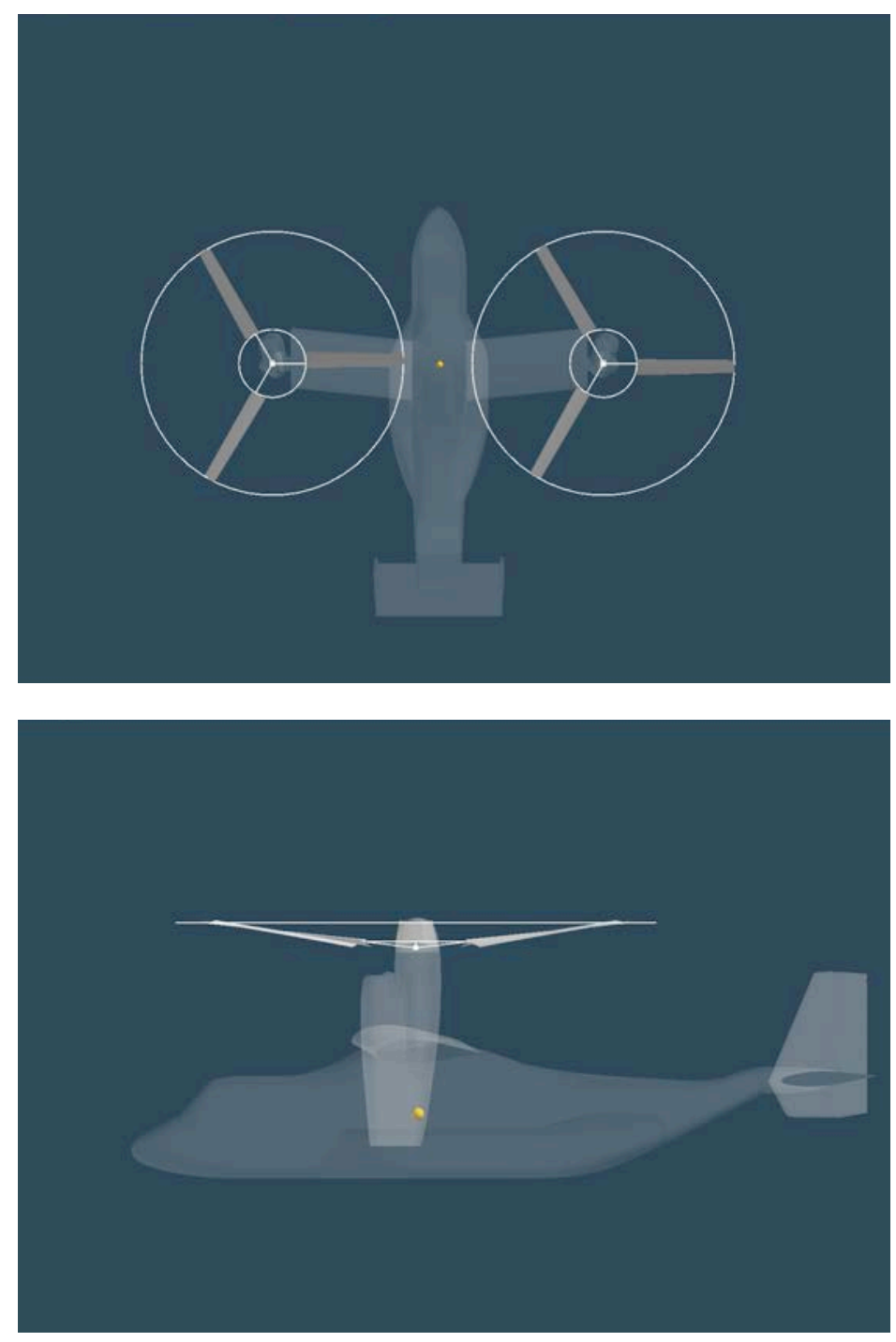

Figure 27. Gold sphere represents model one-forty-eighthscale V-22 tiltrotor aircraft moment center 
Figure 28 represents fuselage force and moment time step histories showing nominal solution convergence (quasi-steady forces and moments reached) has been achieved.
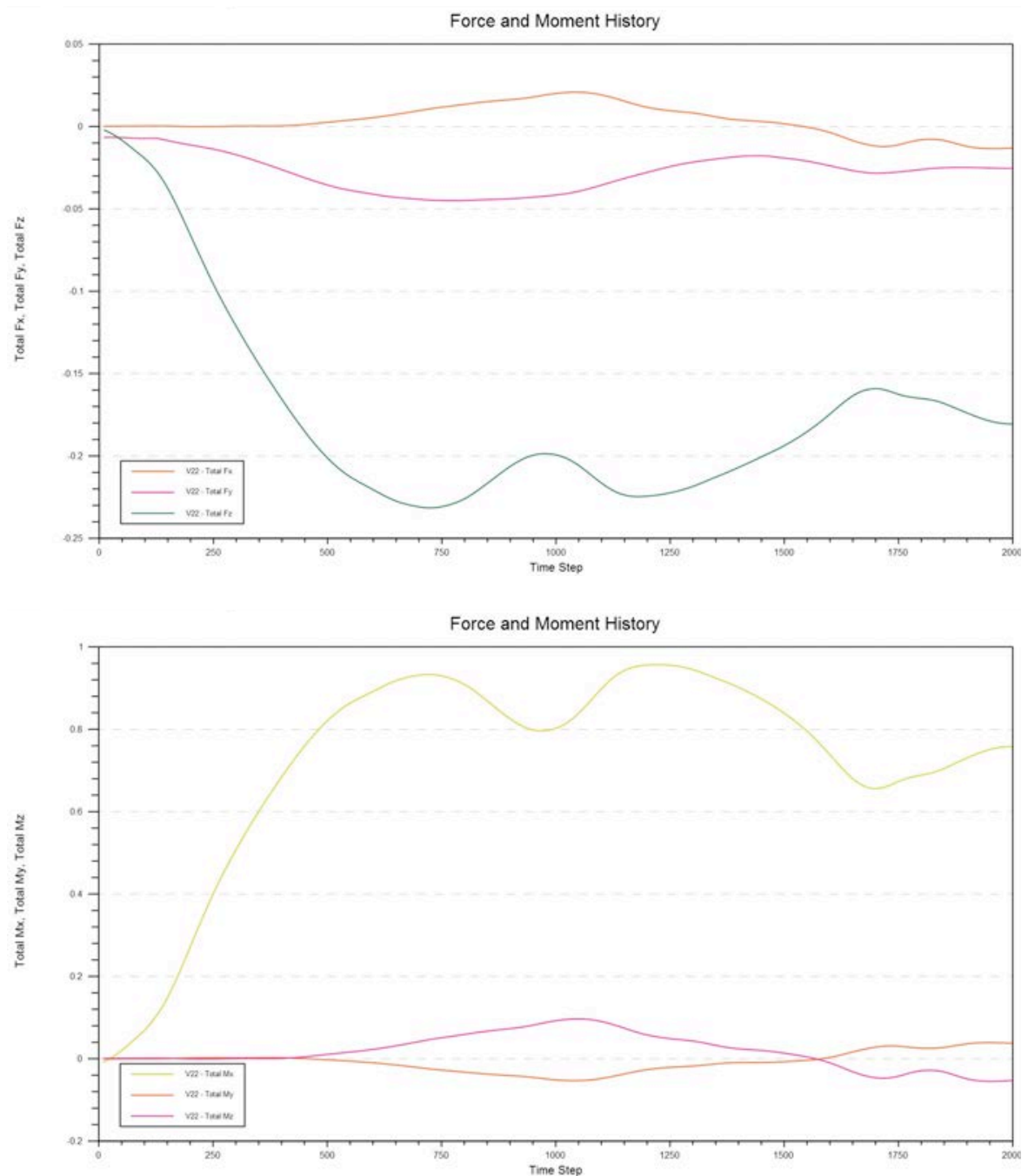

Figure 28. Representative force and moment time step histories for nominal solution convergence (forces in $\mathrm{Ib}_{\mathrm{f}}$ and moments in $\mathrm{ft}_{-} \mathrm{lb}_{\mathrm{f}}$ )

Convergence for the fuselage forces and moments were generally achieved for most of the run cases performed during this study for the simulated twenty seconds. Figure 28 is a representative illustration of the fuselage forces and moments having generally "plateaued" and yielding quasi-steady values by the close of the twenty seconds of simulated time. 
Survey $z / R=2$ and $x / R=3.84$

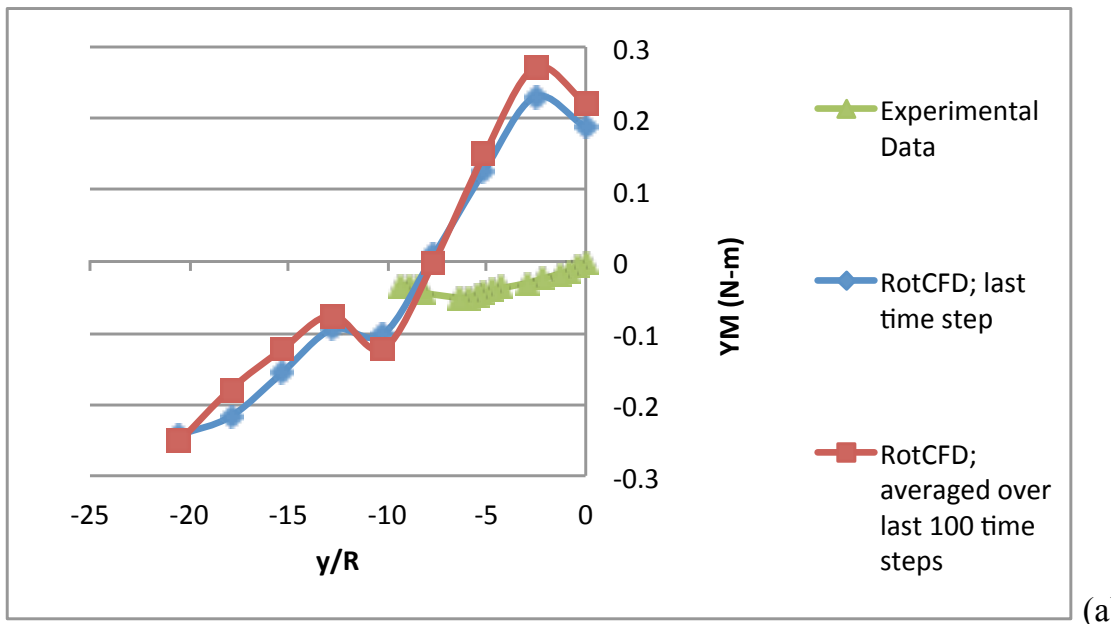

(a)

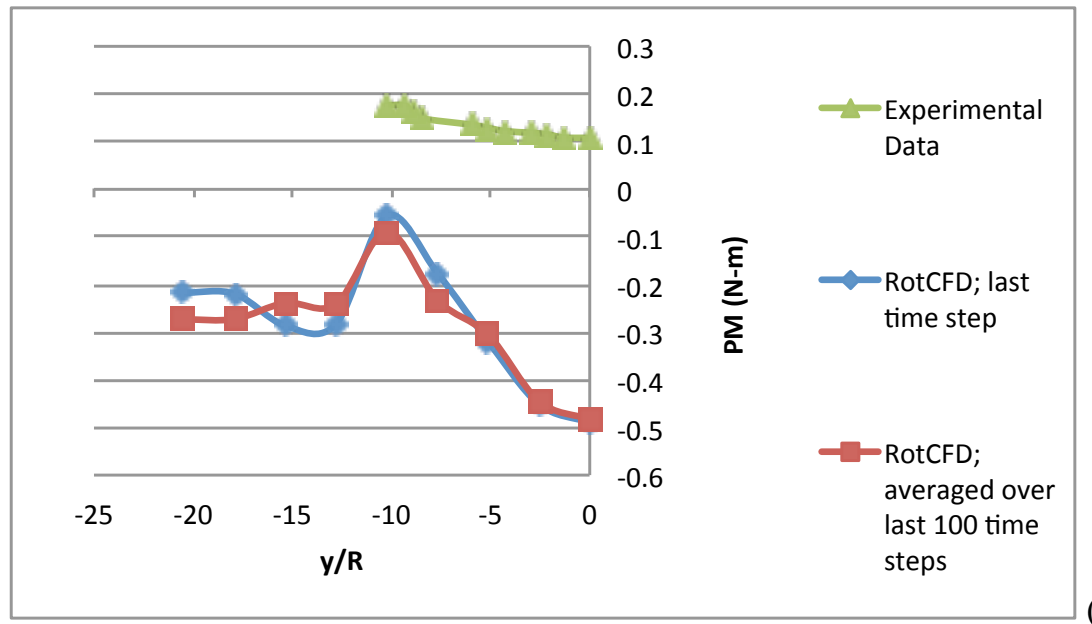

(b)
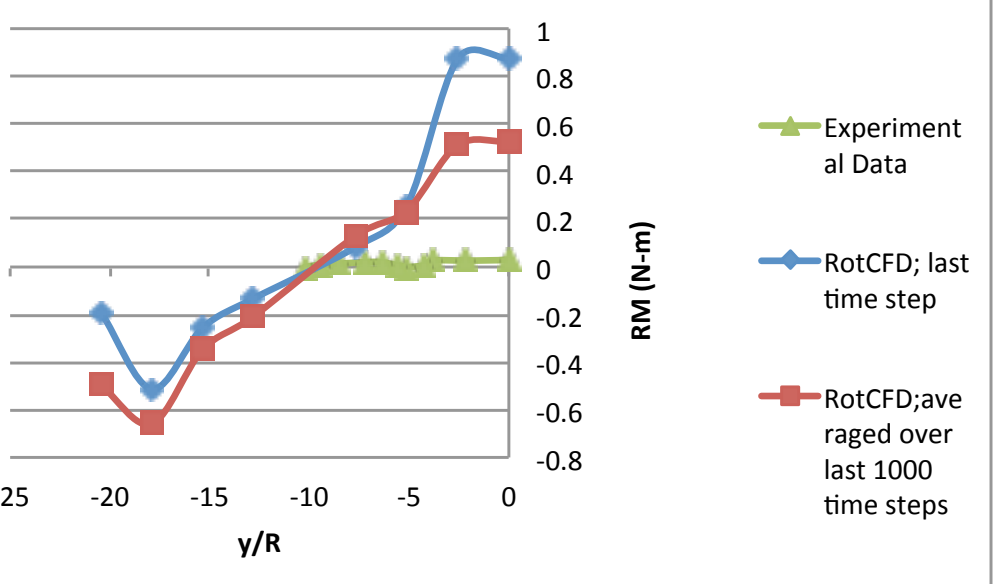

(c)

Figure 29a-c. RotCFD (combined rotors and fuselage forces and moments) comparison with experimental data ( $x / R=3.84)$ : (a) Yawing Moment; (b) Pitching Moment; (c) Rolling Moment 

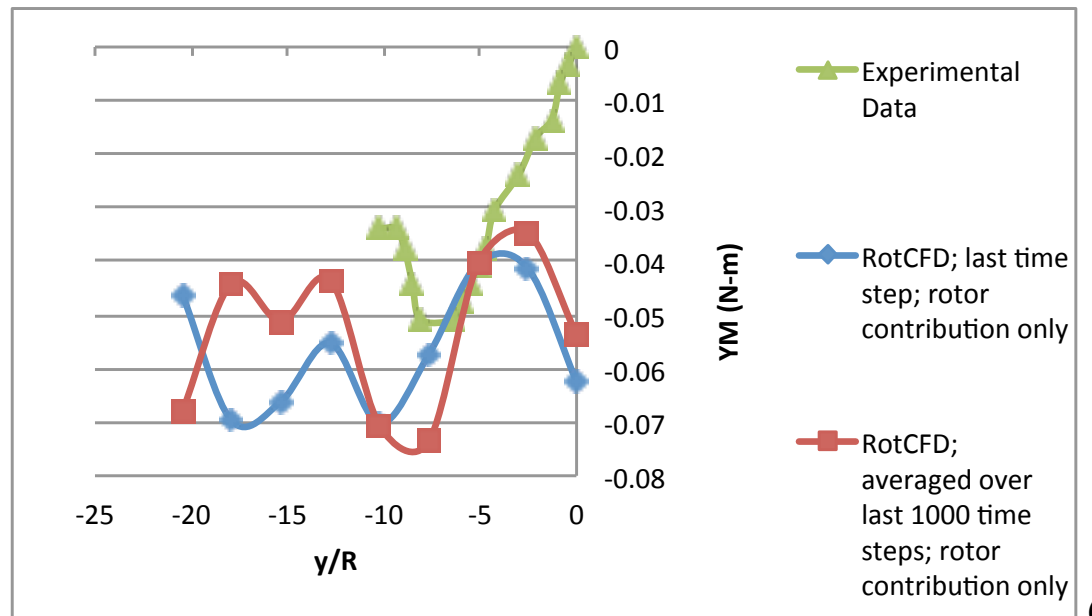

(a)

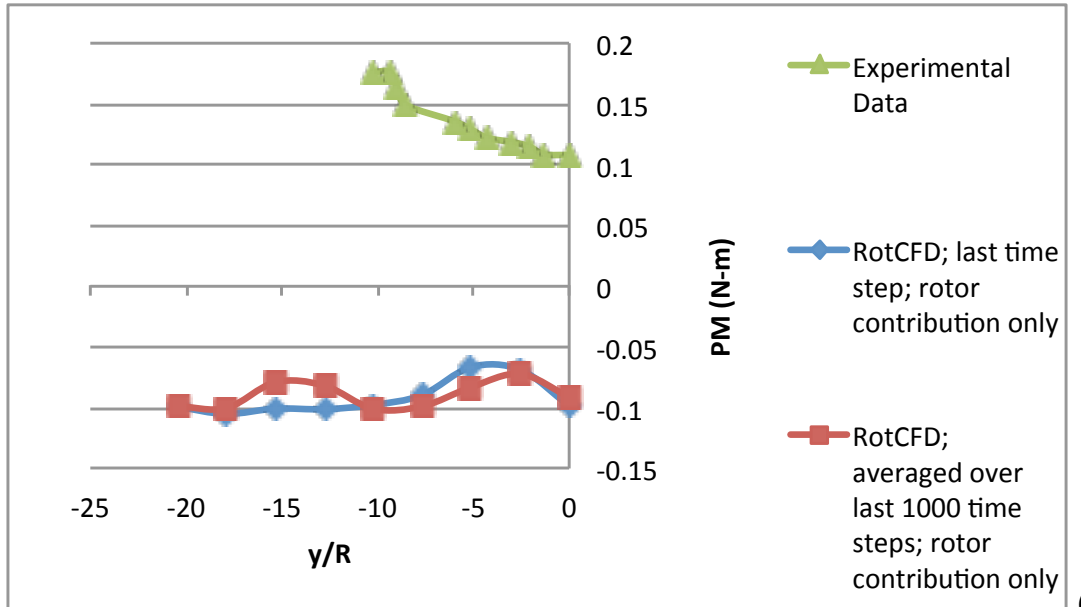

(b)

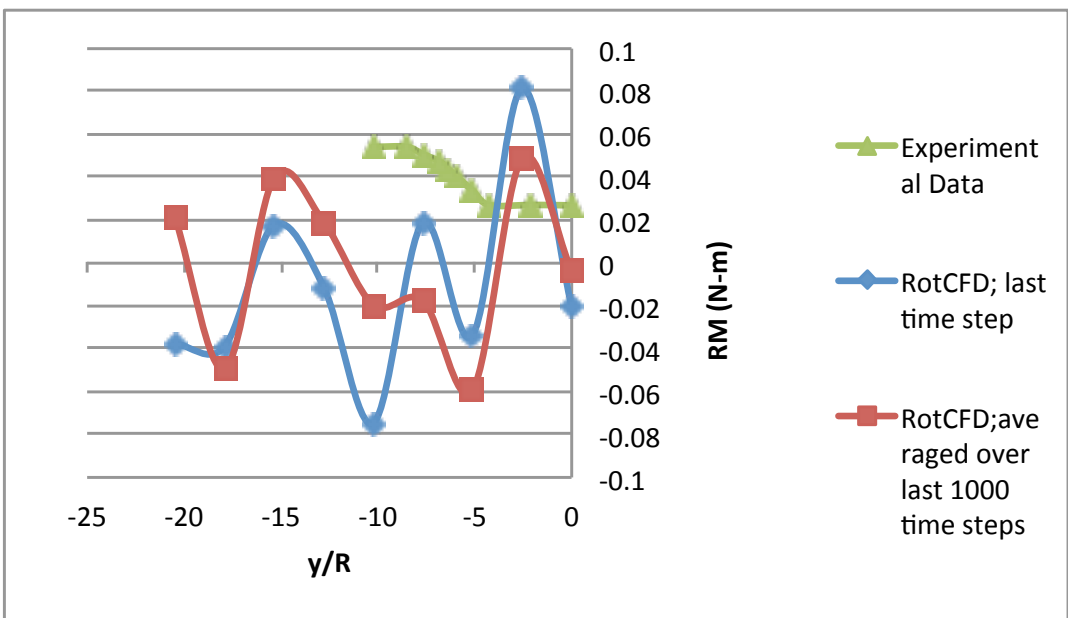

(c)

Figure 30a-c. RotCFD (rotors-only contribution forces and moments) comparison with experimental data ( $x / R=3.84)$ : (a) Yawing Moment; (b) Pitching Moment; (c) Rolling Moment 


\section{Survey $z / R=2$ and $x / R=7.67$}
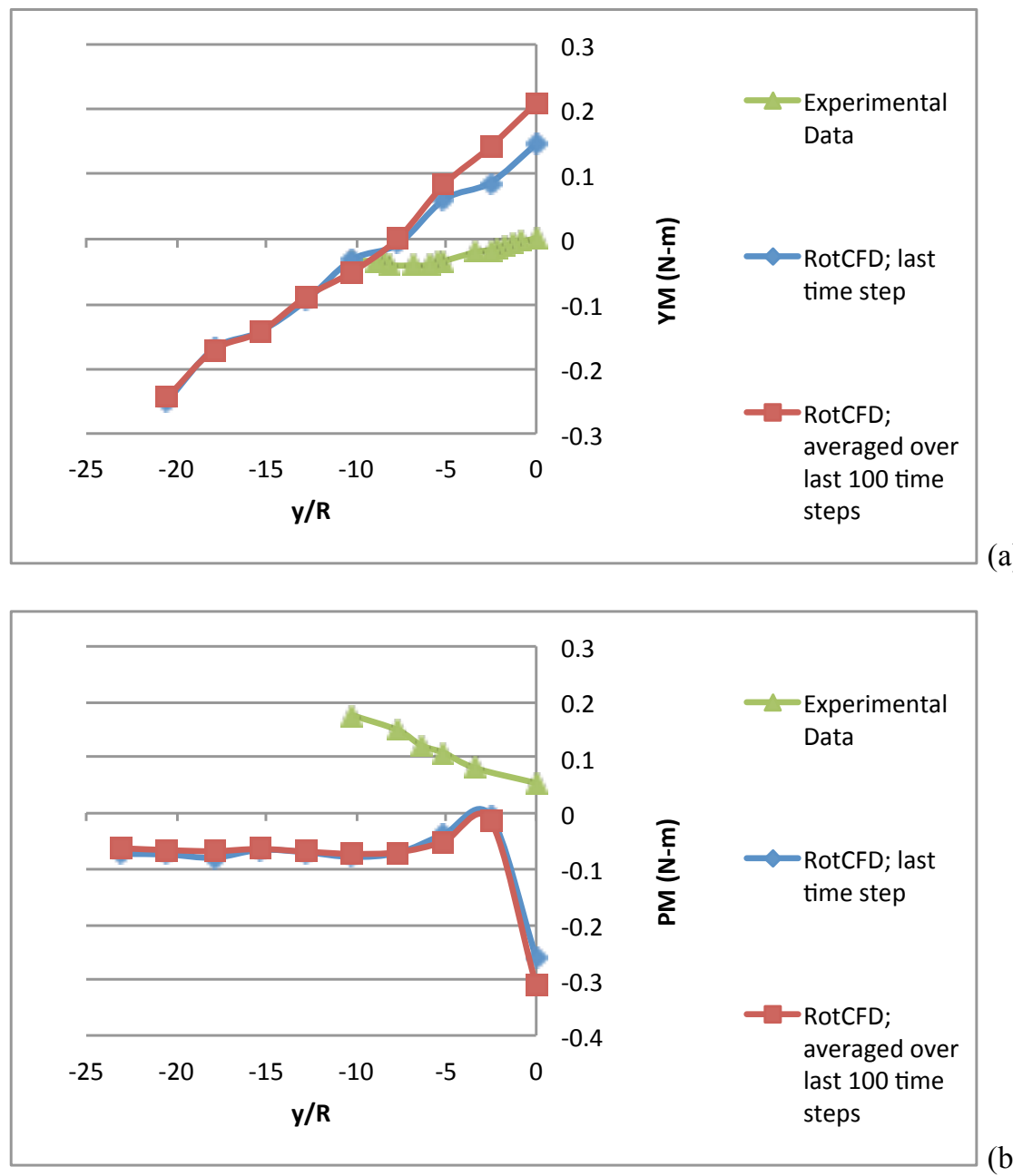

(b)

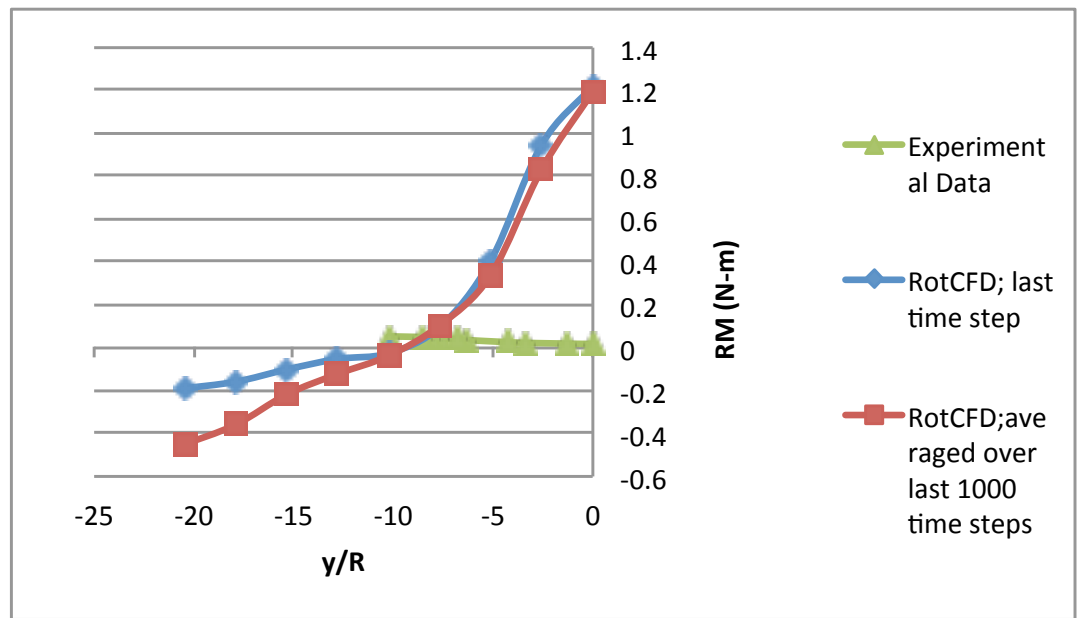

(c)

Figure 31a-c. RotCFD (combined rotors and fuselage forces and moments) comparison with experimental data ( $x / R=7.67)$ : (a) Yawing Moment; (b) Pitching Moment; (c) Rolling Moment 


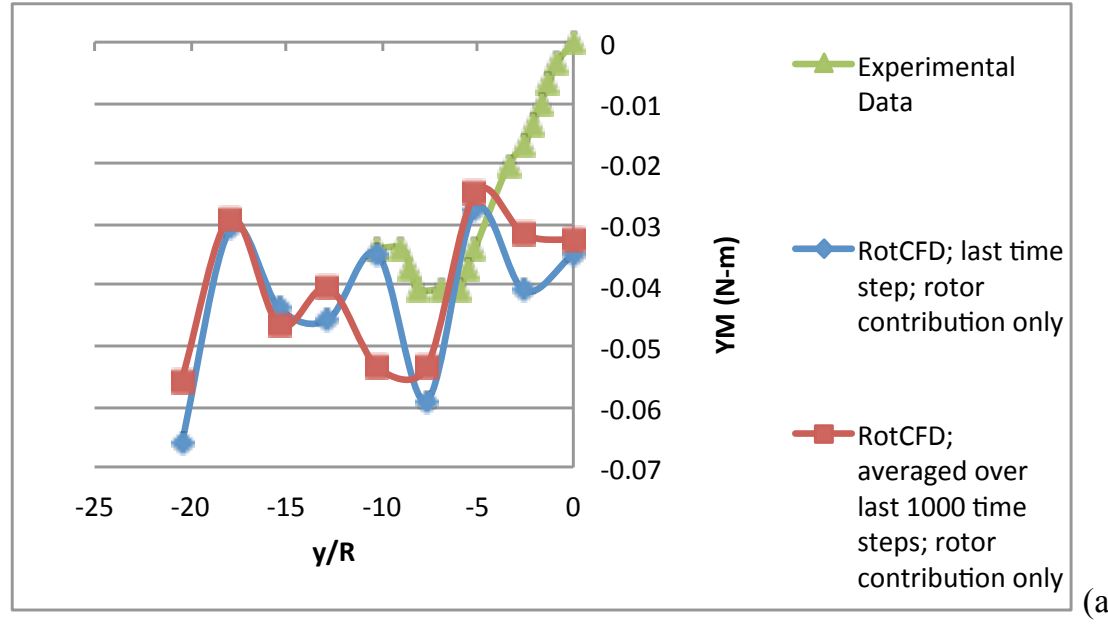

(a)

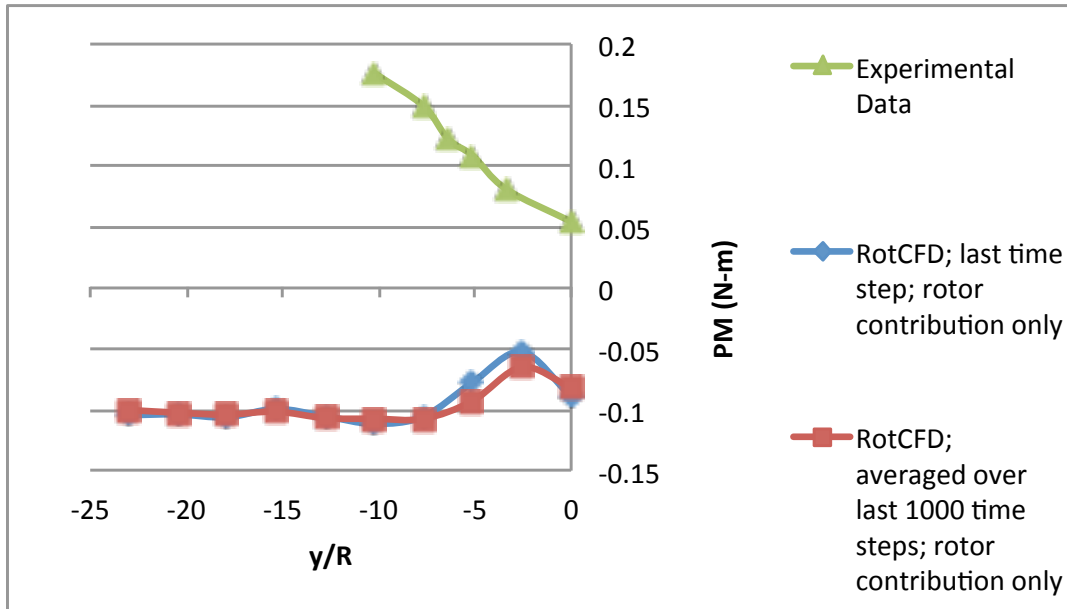

(b)

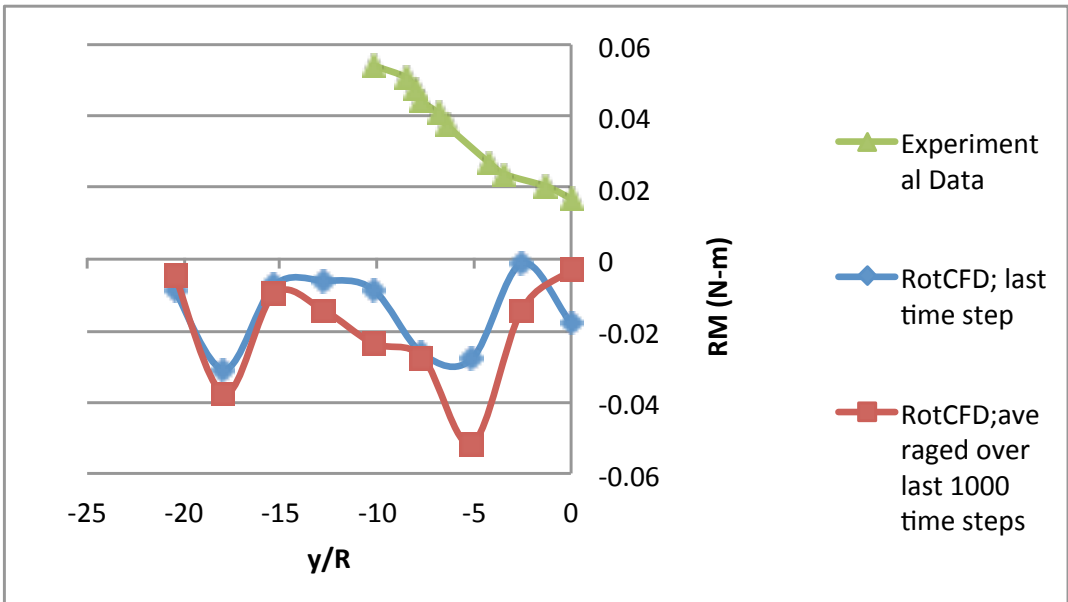

(c)

Figure 32a-c. RotCFD (rotors-only contribution to forces and moments) comparison with experimental data (x/R=7.67): (a) Yawing Moment; (b) Pitching Moment; (c) Rolling Moment

American Institute of Aeronautics and Astronautics 
Survey $z / R=2$ and $x / R=11.51$
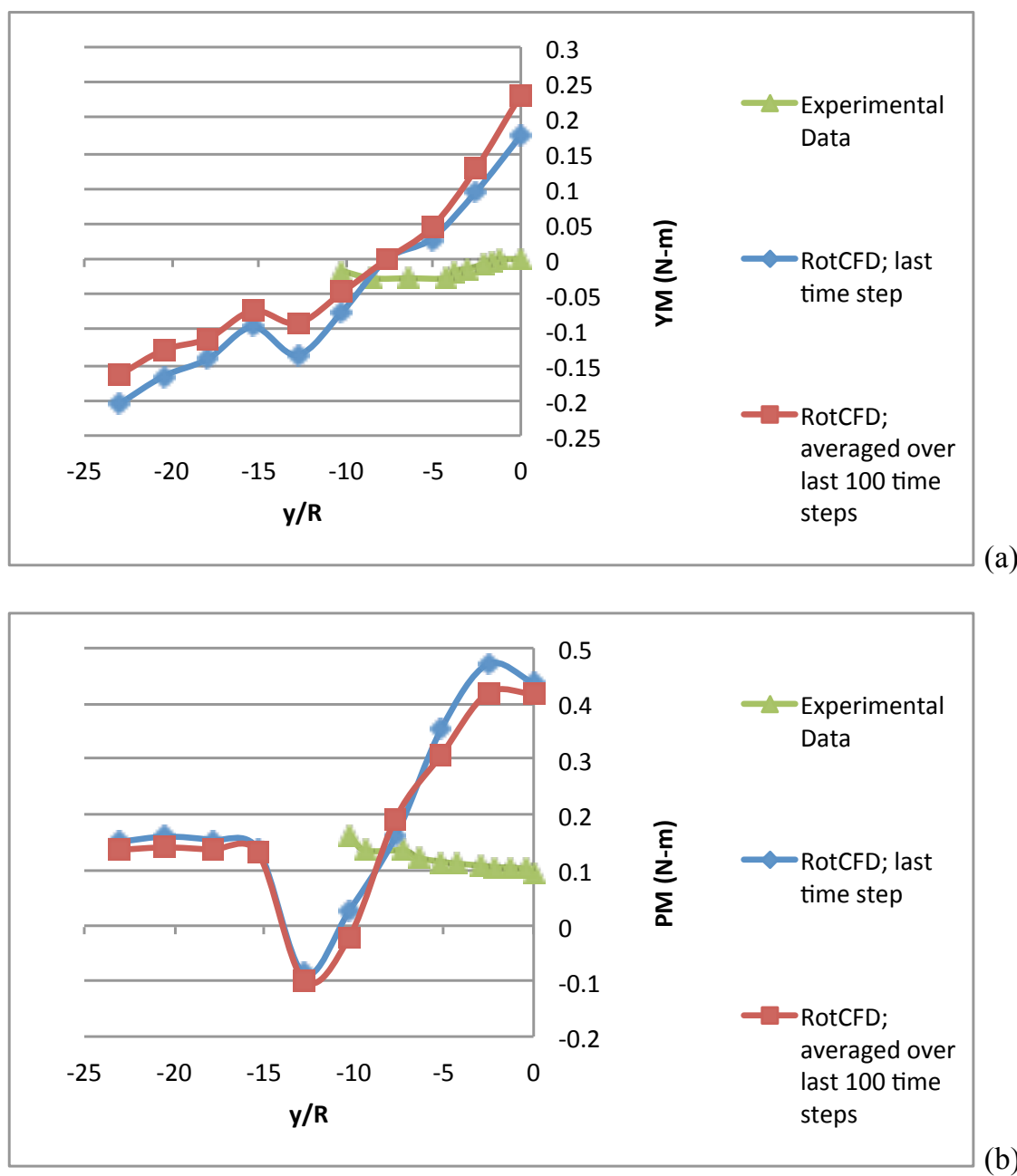

(b)

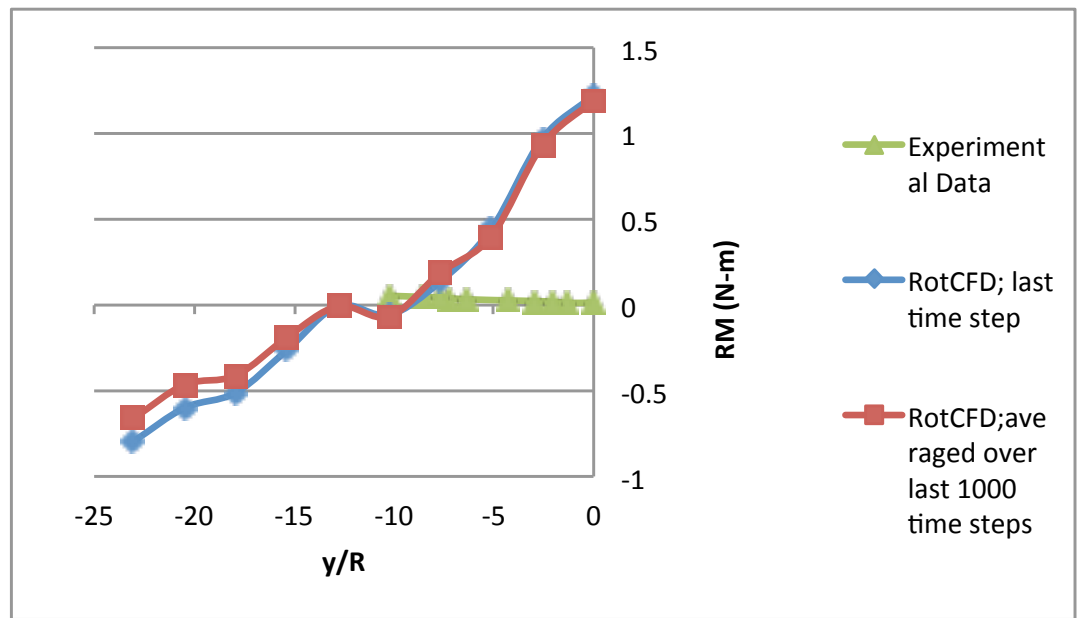

(c)

Figure 33a-c. RotCFD (combined rotors and fuselage forces and moments) comparison with experimental data ( $\mathrm{x} / \mathrm{R}=11.51$ ): (a) Yawing Moment; (b) Pitching Moment; (c) Rolling Moment 

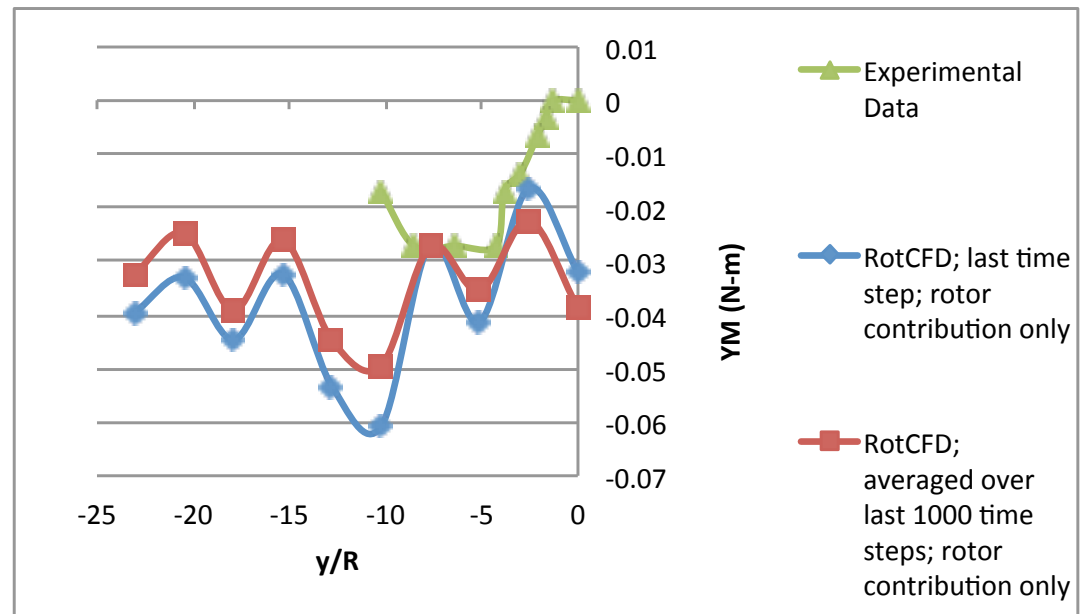

(a)

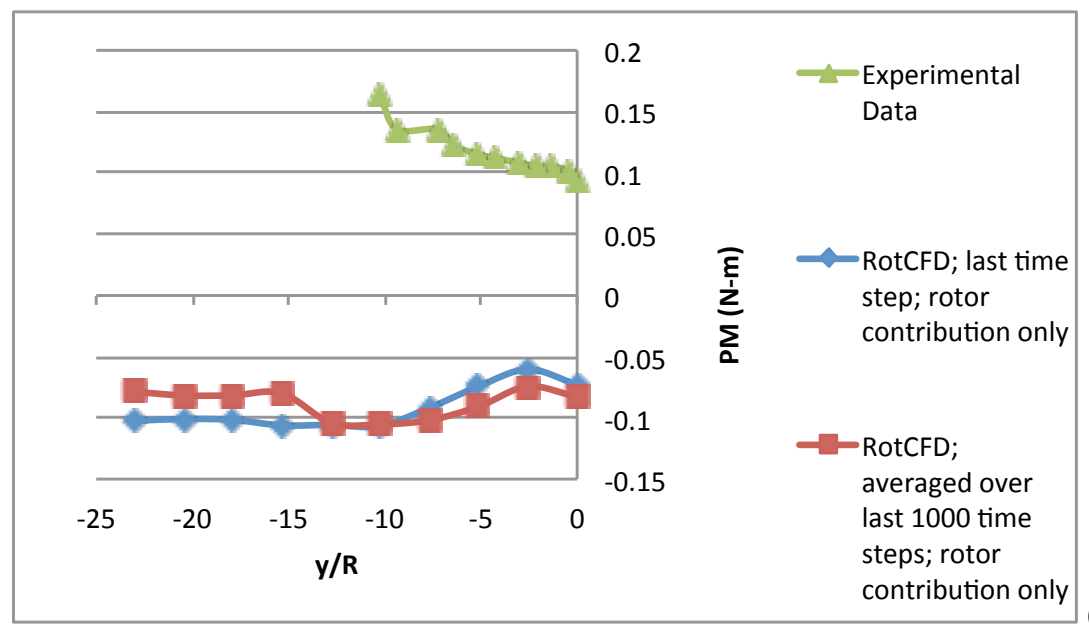

(b)

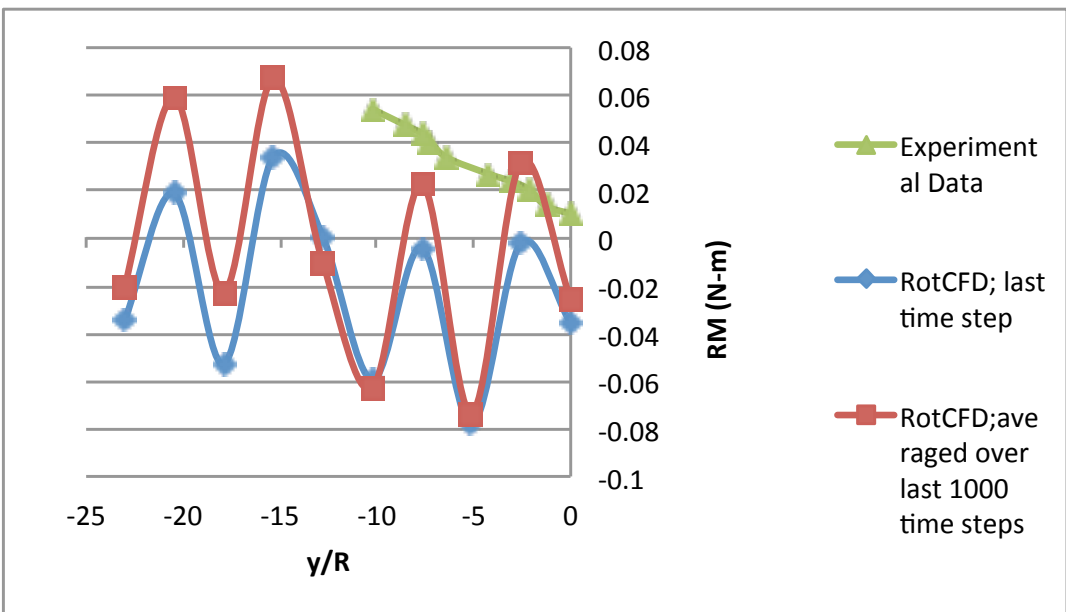

(c)

Figure 34a-c. RotCFD (rotors-only contribution forces and moments) comparison with experimental data (x/R=11.51): (a) Yawing Moment; (b) Pitching Moment; (c) Rolling Moment 
Survey $z / R=2$ and $x / R=15.35$
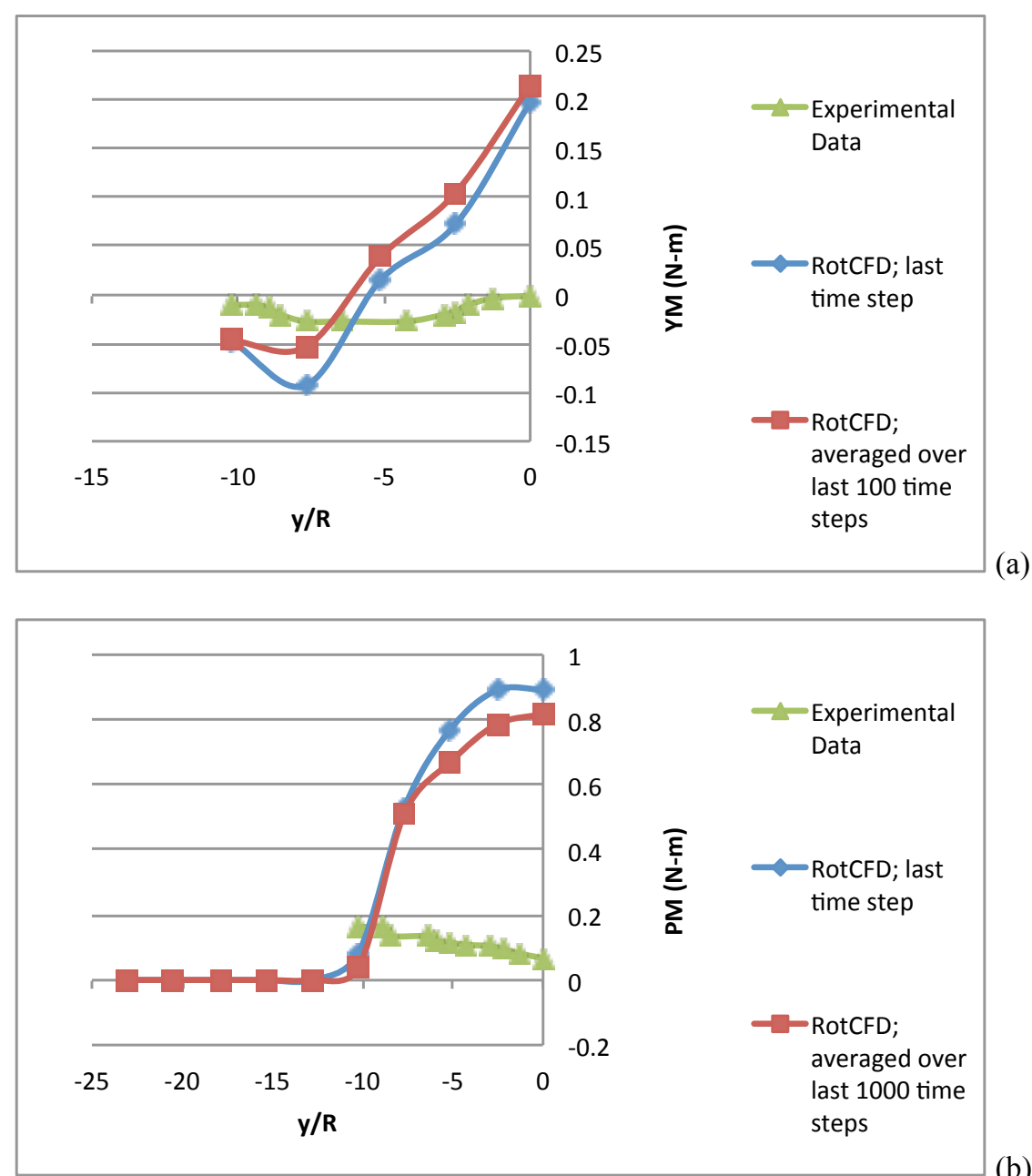

(b)

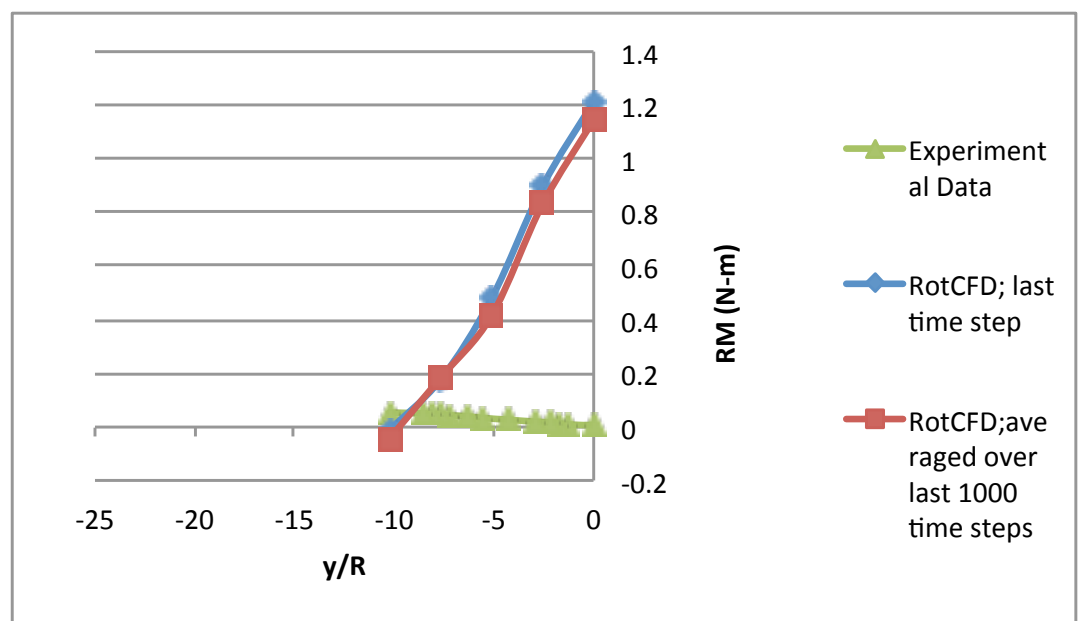

(c)

Figure 35a-c. RotCFD (combined rotors and fuselage forces and moments) comparison with experimental data ( $x / R=15.35$ ): (a) Yawing Moment; (b) Pitching Moment; (c) Rolling Moment 

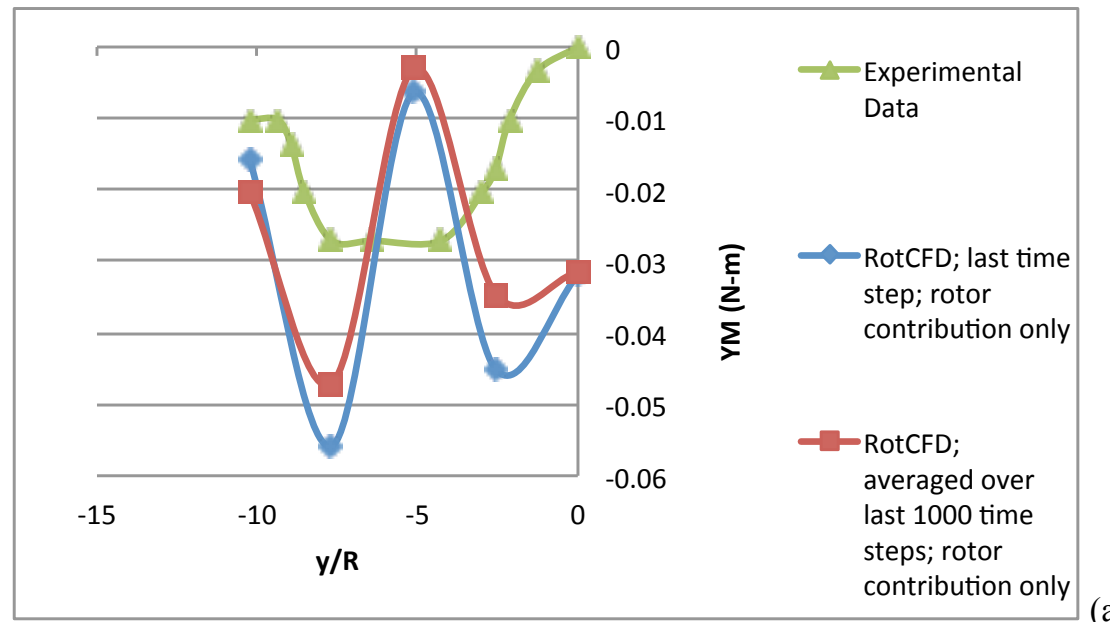

(a)
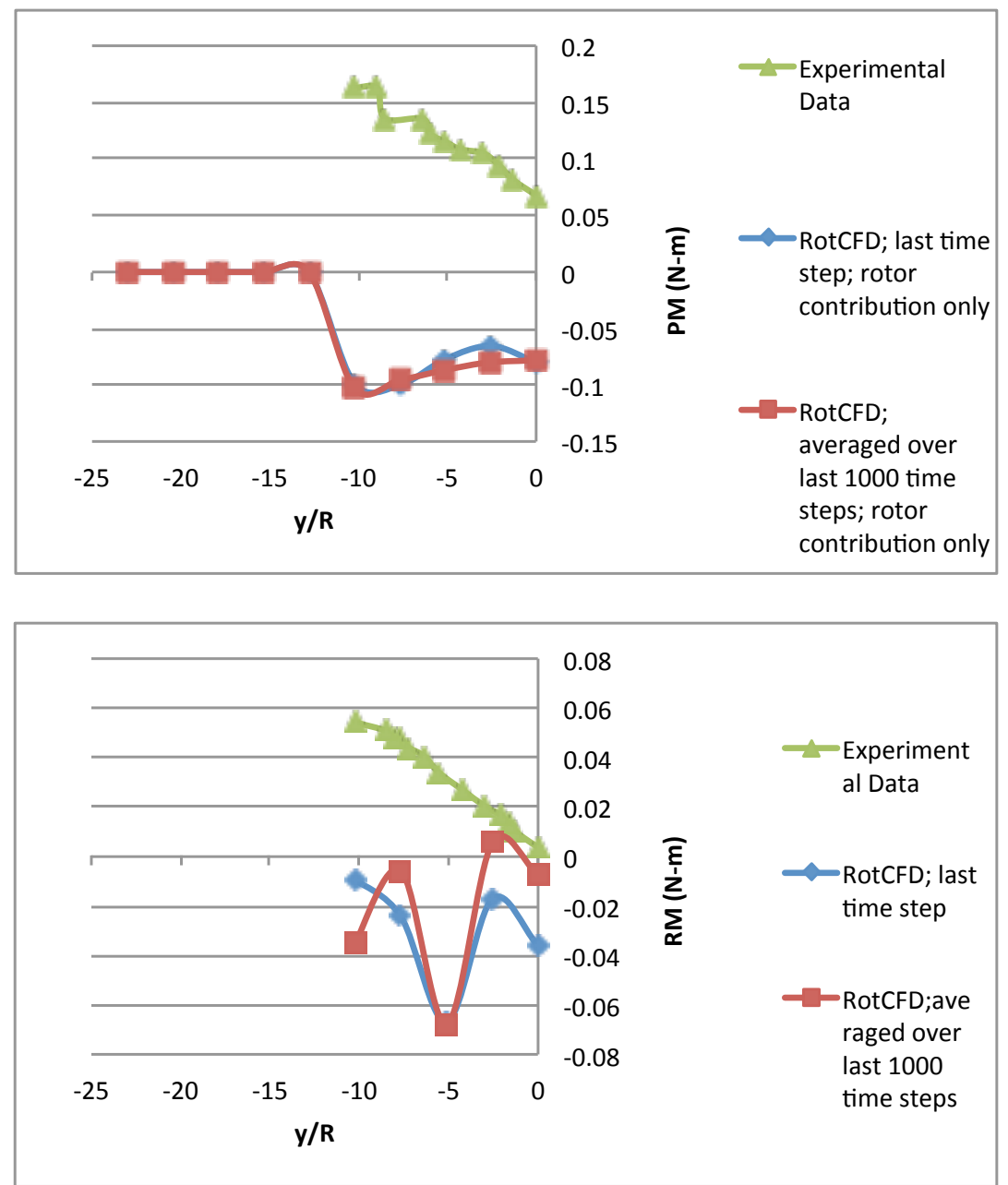

(c)

Figure 36a-c. RotCFD (rotors-only contribution forces and moments) comparison with experimental data (x/R=15.35): (a) Yawing Moment; (b) Pitching Moment; (c) Rolling Moment 